\title{
Derivatives of Eisenstein series and Faltings heights
}

\author{
Stephen S. Kudla, Michael Rapoport and Tonghai Yang
}

\begin{abstract}
We prove a relation between a generating series for the heights of Heegner cycles on the arithmetic surface associated with a Shimura curve and the second term in the Laurent expansion at $s=\frac{1}{2}$ of an Eisenstein series of weight $\frac{3}{2}$ for $\mathrm{SL}(2)$. On the geometric side, a typical coefficient of the generating series involves the Faltings heights of abelian surfaces isogenous to a product of CM elliptic curves, an archimedean contribution, and contributions from vertical components in the fibers of bad reduction. On the analytic side, these terms arise via the derivatives of local Whittaker functions. It should be noted that $s=\frac{1}{2}$ is not the central point for the functional equation of the Eisenstein series in question. Moreover, the first term of the Laurent expansion at $s=\frac{1}{2}$ coincides with the generating function for the degrees of the Heegner cycles on the generic fiber and, in particular, does not vanish.
\end{abstract}

Introduction

Contents

Part I. Arithmetic geometry 888

1 The moduli stack $\mathcal{M}$

893

2 Uniformization

893

3 The Hodge bundle

893

4 The arithmetic Picard group and the arithmetic Chow group

895

5 Special cycles and the generating function

897

899

Part II. Eisenstein series

901

6 Eisenstein series of weight $\frac{3}{2}$

901

7 The main identities

905

8 Fourier expansions and derivatives $\quad 906$

Part III. Computations: geometric $\quad 915$

9 The geometry of $\mathcal{Z}(m) \quad 915$

10 Contributions of horizontal components $\quad 917$

11 Contributions of vertical components $\quad 930$

Appendix A. The case $p=2 \quad 934$

12 Archimedean contributions $\quad 938$

$\begin{array}{ll}\text { Part IV. Computations: analytic } & 941\end{array}$

13 Local Whittaker functions: the non-archimedean case $\quad 941$

14 Local Whittaker functions: the archimedean case $\quad 945$

$\begin{array}{ll}15 \text { The functional equation } & 947\end{array}$

Received 29 April 2002, accepted in final form 24 April 2003.

2000 Mathematics Subject Classification 11G18, 14G40, 11F30 (primary) 11G50, 14G35, $11 \mathrm{~F} 37$ (secondary).

Keywords: Eisenstein series, arithmetic geometry, Shimura curves, heights.

The first author was partially supported by NSF grant DMS-9970506 and by a Max-Planck Research Prize from the Max-Planck Society and Alexander von Humboldt Stiftung. The third author was partially supported by NSF grant DMS-0070476.

This journal is (C) Foundation Compositio Mathematica 2004. 


\section{S. S. Kudla, M. RAPOpORT AND T. YANG}

\section{Introduction}

In a series of papers [Kud97b, KR00b, KR99, KR00a, KRY99, Kud02a], we showed that certain quantities from the arithmetic geometry of Shimura varieties associated to orthogonal groups occur in the Fourier coefficients of the derivative of suitable Siegel-Eisenstein series. It was essential in these examples that this derivative was the second term in the Laurent expansion of a SiegelEisenstein series at the center of symmetry, and that the first term in this Laurent expansion vanished (incoherent case). In the present paper, we prove a relation between a generating function for the heights of Heegner cycles on the arithmetic surface associated with a Shimura curve and the second term in the Laurent expansion at $s=\frac{1}{2}$ of an Eisenstein series of weight $\frac{3}{2}$ for $S L_{2}$. It is remarkable that $s=\frac{1}{2}$ is not the center of symmetry and that the first term of the Laurent expansion is non-zero. In fact, this non-zero value has a geometric interpretation in terms of the Shimura curve over the field of complex numbers. Considering the fact that the Eisenstein series is a rather familiar classical object, it is surprising that this interpretation of its Laurent expansion at $s=\frac{1}{2}$ has not been noticed before. As we argue below, we believe that our result is part of a general pattern involving the heights of divisors on arithmetic models of Shimura varieties associated with orthogonal groups.

We now describe our results in more detail.

Let $B$ be an indefinite division quaternion algebra over $\mathbb{Q}$ and let $O_{B}$ be a maximal order in $B$. Let $D(B)$ be the product of all primes $p$ at which $B$ is division. Let $\mathcal{M}$ be the moduli space of abelian varieties of dimension 2 with a (special) action of $O_{B}$. Then $\mathcal{M}$ is an integral model of the Shimura curve attached to $B$; it is proper of relative dimension 1 over $\operatorname{Spec} \mathbb{Z}$, with semi-stable reduction at all primes and is smooth at all primes $p$ at which $B$ splits, i.e. for $p \nmid D(B)$. Ignoring, for the moment, the fact that $\mathcal{M}$ is only a stack, we may consider $\mathcal{M}$ as an arithmetic surface in the sense of Arakelov theory [Fal83, Bos99].

For each $m \in \mathbb{Z}$ and for $v \in \mathbb{R}_{+}^{\times}$, we define a class in the arithmetic Chow group

$$
\hat{\mathcal{Z}}(m, v)=(\mathcal{Z}(m), \Xi(m, v)) \in \widehat{\mathrm{CH}}^{1}(\mathcal{M}) .
$$

Here, for $m>0, \mathcal{Z}(m)$ is the divisor on $\mathcal{M}$ corresponding to those $O_{B}$-abelian varieties which admit a special endomorphism $x$ with $x^{2}=-m$. These cycles can be viewed as the Shimura curve analogues of the cycles on the modular curve defined by elliptic curves with CM by the order $\mathbb{Z}[\sqrt{-m}]$. For $m<0, \mathcal{Z}(m)=\emptyset$. For all $m \neq 0, \Xi(m, v)$ is the (non-standard) Green's function introduced in [Kud97b]. The class $\hat{\mathcal{Z}}(0, v)$ is defined as follows.

The moduli stack $\mathcal{M}$ carries a universal abelian variety $\mathcal{A} / \mathcal{M}$, and the Hodge bundle $\omega$ on $\mathcal{M}$ is defined by

$$
\omega=\wedge^{2}(\operatorname{Lie}(\mathcal{A}))^{*} .
$$

We equip $\omega$ with the metric $\|\cdot\|$ which, for $z \in \mathcal{M}(\mathbb{C})$ is given by

$$
\|\alpha\|_{z}^{2}=e^{-2 C} \cdot \frac{1}{4 \pi^{2}}\left|\int_{A_{z}(\mathbb{C})} \alpha \wedge \bar{\alpha}\right|
$$

where

$$
C=\frac{1}{2}(\log (4 \pi)+\gamma),
$$

for Euler's constant $\gamma$. The reason for this normalization is explained below. We thus obtain a class $\hat{\omega}=(\omega,\|\cdot\|) \in \widehat{\operatorname{Pic}}(\mathcal{M})$. Also, writing $\hat{\omega}$ for the image of this class in $\widehat{\mathrm{CH}}^{1}(\mathcal{M})$, we set

$$
\hat{\mathcal{Z}}(0, v)=-\hat{\omega}-(0, \log (v))+(0, \mathbf{c}) \in \widehat{\mathrm{CH}}^{1}(\mathcal{M}),
$$

for a constant $\mathbf{c}$ which is defined in (0.15) below. 


\section{DeRivatives of Eisenstein SERIES AND FALtings Heights}

Using the Gillet-Soulé height pairing $\langle\cdot, \cdot\rangle$ between $\widehat{\mathrm{CH}}^{1}(\mathcal{M})$ and $\widehat{\mathrm{Pic}}(\mathcal{M})$ [GS90], we form the height generating series

$$
\phi_{\text {height }}(\tau)=\sum_{m}\langle\hat{\mathcal{Z}}(m, v), \hat{\omega}\rangle q^{m},
$$

where, for $\tau=u+i v$ in the upper half plane $\mathfrak{H}$, we have set $q=e(\tau)=e^{2 \pi i \tau}$. The quantities $\langle\hat{\mathcal{Z}}(m, v), \hat{\omega}\rangle$ can be thought of as arithmetic degrees [BGS94, Bos91]. At the same time, we can define the more elementary generating series

$$
\phi_{\operatorname{deg}}(\tau)=\sum_{m} \operatorname{deg}(\hat{\mathcal{Z}}(m, v)) q^{m}
$$

where $\operatorname{deg}(\hat{\mathcal{Z}}(m, v))=\operatorname{deg}(\mathcal{Z}(m))$ is simply the usual (geometric) degree of the 0 -cycle $\mathcal{Z}(m)_{\mathbb{C}}$ on the complex Shimura curve $\mathcal{M}_{\mathbb{C}}$.

To see that these generating series are the $q$-expansions of modular forms, we consider a family of Eisenstein series. In 1975, Zagier [Zag75], (see also [Coh75]) introduced a (non-holomorphic) Eisenstein series of weight $\frac{3}{2}$, whose Fourier expansion is given by

$$
\mathcal{F}(\tau)=-\frac{1}{12}+\sum_{m>0} H(m) q^{m}+\sum_{n} \frac{1}{16 \pi} v^{-\frac{1}{2}} \int_{1}^{\infty} e^{-4 \pi n^{2} v r} r^{-\frac{3}{2}} d r q^{-n^{2}},
$$

where $H(m)$ is the number of classes of positive definite integral binary quadratic forms of discriminant $-m$. This series, which played a key role in the work of Hirzebruch and Zagier [HZ76] on generating functions for intersection numbers of curves on Hilbert modular surfaces, can be viewed as the value at $s=\frac{1}{2}$ of an Eisenstein series $\mathcal{F}(\tau, s)$, defined for $s \in \mathbb{C}$ and satisfying a functional equation $\mathcal{F}(\tau,-s)=\mathcal{F}(\tau, s)$. In fact, there is a whole family of such series, $\mathcal{E}(\tau, s ; D)$, where $D$ is a square free positive integer, whose values at $s=\frac{1}{2}$, for $D>1$, are given by ${ }^{1}$

$$
\mathcal{E}\left(\tau, \frac{1}{2} ; D\right)=-(-1)^{\operatorname{ord}(D)} \frac{1}{12} \prod_{p \mid D}(p-1)+\sum_{m>0} 2 \delta(d ; D) H_{0}(m ; D) q^{m} .
$$

Here $H_{0}(m ; D)$ is a variant of the class number $H(m), \delta(d ; D)$ is either zero or a power of two (cf. (8.17) and (8.18), respectively), and $\operatorname{ord}(D)$ is the number of prime factors of $D$. In the case $D=D(B)>1$, a simple calculation of $\operatorname{deg}(\mathcal{Z}(m))$ proves the (known) relation

$$
\phi_{\operatorname{deg}}(\tau)=\mathcal{E}\left(\tau, \frac{1}{2} ; D(B)\right),
$$

so that the value of $\mathcal{E}(\tau, s ; D(B))$ at $s=\frac{1}{2}$ is the degree generating function. The main result of this paper asserts that the second term in the Laurent expansion of the Eisenstein series $\mathcal{E}(\tau, s ; D(B))$ at the point $s=\frac{1}{2}$ contains information about the arithmetic surface $\mathcal{M}$ :

Theorem A. For $D(B)>1$,

$$
\phi_{\text {height }}(\tau)=\mathcal{E}^{\prime}\left(\tau, \frac{1}{2} ; D(B)\right)
$$

This identity is proved by a direct computation of the two sides. The resulting formulas (cf. Theorem 8.8) are quite complicated. For example, for $m>0$ in the case in which $\operatorname{deg}(\mathcal{Z}(m) \mathbb{C}) \neq 0$,

\footnotetext{
${ }^{1}$ Our normalization of these series differs slightly at the prime 2 from that used by Zagier, so that our $\mathcal{E}\left(\tau, \frac{1}{2} ; 1\right)$ is not quite Zagier's function, cf. (8.23) below.
} 


\section{S. S. Kudla, M. Rapoport And T. YAng}

the coefficient of $q^{m}$ in the derivative of the Eisenstein series is given by

$$
\begin{aligned}
2 \delta(d ; D(B)) H_{0}(m ; D(B)) \cdot\left[\frac{1}{2} \log (d)+\frac{L^{\prime}\left(1, \chi_{d}\right)}{L\left(1, \chi_{d}\right)}-\frac{1}{2} \log (\pi)-\frac{1}{2} \gamma\right. \\
\left.+\frac{1}{2} J(4 \pi m v)+\sum_{\substack{p \\
p \nmid D(B)}}\left(\log |n|_{p}-\frac{b_{p}^{\prime}(n, 0 ; D)}{b_{p}(n, 0 ; D)}\right)+\sum_{\substack{p \\
p \mid D(B)}} K_{p} \log (p)\right] .
\end{aligned}
$$

Here we write the discriminant of the order $\mathbb{Z}[\sqrt{-m}]$ as $4 m=n^{2} d$ for a fundamental discriminant $-d$, and the other notation is explained in Theorem 8.8. Theorem A asserts that this expression coincides with the height pairing $\langle\hat{\mathcal{Z}}(m, v), \hat{\omega}\rangle$ ! A point over $\overline{\mathbb{Q}}$ of $\mathcal{Z}(m)$ corresponds to an $O_{B}$-abelian surface $A$ over $\overline{\mathbb{Q}}$, equipped with an action of $\mathbb{Z}[\sqrt{-m}]$ commuting with that of $O_{B}$. Such a surface is isogenous to a product $E_{d} \times E_{d}$, where $E_{d}$ is an elliptic curve with complex multiplication by the maximal order $O_{k}$ in the imaginary quadratic field $\mathbb{Q}(\sqrt{-d})$. With our normalization of the metric on the Hodge bundle, the Faltings height of $E_{d} \times E_{d}$ is given by

$$
h_{\mathrm{Fal}}^{*}\left(E_{d} \times E_{d}\right)=2 h_{\mathrm{Fal}}^{*}\left(E_{d}\right)=\frac{1}{2} \log (d)+\frac{L^{\prime}\left(1, \chi_{d}\right)}{L\left(1, \chi_{d}\right)}-\frac{1}{2} \log (\pi)-\frac{1}{2} \gamma,
$$

so that the geometric meaning of the first terms of (0.11), and of our title, emerges. The change in the Faltings height due to the isogeny is accounted for by the term involving the sum over $p \nmid D(B)$, where the logarithmic derivatives occurring there are given explicitly in Lemma 8.10. The sum over $p \mid D(B)$ has the following geometric meaning. The arithmetic surface $\mathcal{M}$ has bad reduction at such primes and the cycle $\mathcal{Z}(m)$, defined as a moduli space, can include components of the special fiber $\mathcal{M}_{p}$, i.e. vertical components [KR00a]. Their contribution to the height pairing coincides with the term $K_{p} \cdot \log (p)$ in (0.11), where $K_{p}$ is given explicitly in Theorem 8.8. Finally, there is an additional 'archimedean' term in the height pairing, which arises from the fact that the Green's function $\Xi(m, v)$ is not orthogonal to the Chern form $\mu=c_{1}(\hat{\omega})$ of the Hodge bundle. This contribution coincides with the term involving $\frac{1}{2} J(4 \pi m v)$.

For $m=0$, the constant term of the derivative of the Eisenstein series at $s=\frac{1}{2}$ with $D=$ $D(B)>1$ is given by

$$
\mathcal{E}_{0}^{\prime}\left(\tau, \frac{1}{2} ; D(B)\right)=\zeta_{D}(-1)\left[\frac{1}{2} \log (v)-2 \frac{\zeta^{\prime}(-1)}{\zeta(-1)}-1+2 C+\sum_{p \mid D} \frac{p \log (p)}{p-1}\right],
$$

where $\zeta_{D}(s)=\zeta(s) \prod_{p \mid D}\left(1-p^{-s}\right)$. On the other hand, by (0.5), the constant term of the generating function for heights is given by

$$
\phi_{\text {height }, 0}(\tau)=\langle\hat{\mathcal{Z}}(0, v), \hat{\omega}\rangle=-\langle\hat{\omega}, \hat{\omega}\rangle-\frac{1}{2} \log (v) \operatorname{deg}(\omega)+\frac{1}{2} \mathbf{c} \cdot \operatorname{deg}(\omega) .
$$

Noting that $\operatorname{deg}(\omega)=-\zeta_{D}(-1)$, we see that the constant terms coincide provided that $\mathbf{c}$ has the value determined by the relation

$$
\frac{1}{2} \operatorname{deg}(\hat{\omega}) \cdot \mathbf{c}=\langle\hat{\omega}, \hat{\omega}\rangle-\zeta_{D(B)}(-1)\left[2 \frac{\zeta^{\prime}(-1)}{\zeta(-1)}+1-2 C-\sum_{p \mid D(B)} \frac{p \log (p)}{p-1}\right] .
$$

We take this value for $\mathbf{c}$ in our definition of $\hat{\mathcal{Z}}(0, v)$ above.

In fact, we conjecture that $\mathbf{c}=-\log (D(B))$, i.e. that

$$
\langle\hat{\omega}, \hat{\omega}\rangle \stackrel{? ?}{=} \zeta_{D(B)}(-1)\left[2 \frac{\zeta^{\prime}(-1)}{\zeta(-1)}+1-2 C-\frac{1}{2} \sum_{p \mid D(B)} \frac{p+1}{p-1} \log (p)\right] .
$$

If we write $\hat{\omega}_{o}=\left(\omega,\|\cdot\|_{\text {nat }}\right)$ for the Hodge bundle with the more standard choice of metric (cf. (3.11) below and [Bos99]), and if we delete the 'extra' factor of $\frac{1}{2}$ which occurs due to the fact that $\mathcal{M}$ is 


\section{DeRivatives of Eisenstein SERIES AND FALtings Heights}

a stack (cf. $\S 4)$, then (0.16) amounts to

$$
\left\langle\hat{\omega}_{o}, \hat{\omega}_{o}\right\rangle^{\text {nat }} \stackrel{? ?}{=} 4 \zeta_{D}(-1)\left[\frac{\zeta^{\prime}(-1)}{\zeta(-1)}+\frac{1}{2}-\frac{1}{4} \sum_{p \mid D(B)} \frac{p+1}{p-1} \log (p)\right] .
$$

If we formally take $D=D(B)=1$, so that $\mathcal{M}$ would be the modular curve and $\hat{\omega}_{o}$ the bundle of modular forms of weight two with the Petersson metric, then, indeed, by the result of Bost [Bos] and Kühn [Kuh01],

$$
\left\langle\hat{\omega}_{o}, \hat{\omega}_{o}\right\rangle^{\text {nat }}=4 \zeta(-1)\left[\frac{\zeta^{\prime}(-1)}{\zeta(-1)}+\frac{1}{2}\right] .
$$

Thus, (0.16) holds for $D(B)=1$, i.e. in the case of the modular curve $\mathcal{M}$.

We expect that Theorem A will hold when $D(B)=1$ as well [Yan]. There are, however, extra complications. The first is that the metric on $\hat{\omega}$ becomes singular at the cusp. This difficulty was overcome by Bost, [Bos99, Bos] and Kühn [Kuh01], by extending the definition of $\widehat{\operatorname{Pic}}(\mathcal{M}) \simeq \widehat{\mathrm{CH}}^{1}(\mathcal{M})$ to allow more general Green's functions. On these more general Chow groups, the geometric degree map should be defined as

$$
\operatorname{deg}: \widehat{\mathrm{CH}}^{1}(\mathcal{M}) \longrightarrow \mathbb{R}, \quad\left(Z, g_{Z}\right) \mapsto \int_{\mathcal{M}(\mathbb{C})} \omega_{Z},
$$

where $\omega_{Z}$ is the (now not necessarily smooth) form occurring on the right-hand side of the Green's equation

$$
d d^{c} g_{Z}+\delta_{Z}=\omega_{Z}
$$

Note that this definition agrees with the previous one in the case $D(B)>1$. With our previous definition of $\hat{\mathcal{Z}}(m, v)$ for $m \neq 0$ and $m \neq-n^{2}$ and with a slight modification when $m=0$ or $m=-n^{2}$, the result of Funke [Fun99, Fun02] shows that, indeed,

$$
\begin{aligned}
\phi_{\operatorname{deg}}(\tau) & =\mathcal{E}\left(\tau, \frac{1}{2} ; 1\right) \\
& =-\frac{1}{12}+\sum_{m>0} 2 H_{0}(m ; 1) q^{m}+\sum_{n \in \mathbb{Z}} \frac{1}{8 \pi} v^{-\frac{1}{2}} \int_{1}^{\infty} e^{-4 \pi n^{2} v r} r^{-\frac{3}{2}} d r \cdot q^{-n^{2}} .
\end{aligned}
$$

In fact, if we had defined the cycles $\mathcal{Z}(m)$ by imposing the action of an order of discriminant $m$ (rather than $4 m$ ) on our $O_{B}$-abelian surface, then the degree generating function would coincide exactly with Zagier's function (0.8)! We defer the calculations of the additional terms which occur in the derivative $\mathcal{E}^{\prime}\left(\tau, \frac{1}{2} ; 1\right)$ and in the height generating function for $D(B)=1$ to a sequel to this paper $[\mathrm{KRY}]$.

Relations like those proved here between the first (respectively second) term of the Laurent expansion of an Eisenstein series and the generating function for degrees (respectively heights) should hold in much greater generality. More precisely, suppose that $V$ is a rational vector space with non-degenerate inner product of signature $(n, 2)$. Let $H=\operatorname{GSpin}(V)$ and let $D$ be the space of oriented negative two-planes in $V(\mathbb{R})$. Then, for each compact open subgroup $K \subset H\left(\mathbb{A}_{f}\right)$, there is a quasiprojective variety $X_{K}$, defined over $\mathbb{Q}$, with

$$
X_{K}(\mathbb{C}) \simeq H(\mathbb{Q}) \backslash\left(D \times H\left(\mathbb{A}_{f}\right) / K\right) .
$$

For each integer $m>0$, and each $K$-invariant 'weight function' $\varphi \in S\left(V\left(\mathbb{A}_{f}\right)\right)^{K}$ in the Schwartz space of $V\left(\mathbb{A}_{f}\right)$, there is a divisor $Z(m, \varphi)_{K}$ on $X_{K}$, rational over $\mathbb{Q}$ [Kud97a]. The variety $X_{K}$ comes equipped with a metrized line bundle $\hat{\mathcal{L}}=(\mathcal{L},\|\cdot\|)$, and it is proved in [Kud03a] that, with the exception of the cases $n=1$ with $V$ isotropic and $n=2$ with $V$ split, the degree generating function

$$
\phi_{\operatorname{deg}}(\tau ; \varphi):=\operatorname{vol}\left(X_{K}\right) \varphi(0)+\sum_{m>0} \operatorname{deg}(Z(m, \varphi)) q^{m}
$$




\section{S. S. Kudla, M. Rapoport And T. YAng}

coincides with the value $E(\tau, n / 2 ; \varphi)$ of an Eisenstein series $E(\tau, s ; \varphi)$ of weight $n / 2+1$ associated to $\varphi$. Here $\operatorname{vol}\left(X_{K}\right)$ (respectively $\operatorname{deg}(Z(m, \varphi))$ ) is the volume of $X_{K}(\mathbb{C})$ (respectively $Z(m, \varphi)_{K}$ ) with respect to $\Omega^{n}$ (respectively $\Omega^{n-1}$ ), where $\Omega$ is the negative of the first Chern form of $\hat{\mathcal{L}}$. We believe that there should be an analogue of Theorem $\mathrm{A}$ in this situation. To obtain such a result, we need, first of all, suitable extensions $\mathcal{Z}(m ; \varphi)$ of the cycles $Z(m, \varphi)$ to suitable integral models $\mathfrak{X}_{K}$ of the $X_{K}$ 's. Next, since the varieties $X_{K}$ are, in general, not projective, we need nice compactifications $\overline{\mathfrak{X}}_{K}$ and, more importantly, an extension of the Gillet-Soulé theory, general enough to allow the singularities of the metric on the extension $\hat{\omega}$ of $\hat{\mathcal{L}}^{\vee}$ to the compactification, etc. Assuming all of this, we would have cycles

$$
\widehat{\mathcal{Z}}(m, v ; \varphi)=(\mathcal{Z}(m ; \varphi), \Xi(m, v)) \in \widehat{\mathrm{CH}}^{1}(\overline{\mathfrak{X}})_{K}
$$

and a class $\hat{\omega} \in \widehat{\mathrm{CH}}^{1}(\overline{\mathfrak{X}})_{K}$. The analogue of Theorem A would identify the height generating series

$$
\phi_{\text {height }}(\tau ; \varphi):=\sum_{m}\left\langle\widehat{\mathcal{Z}}(m, v ; \varphi), \hat{\omega}^{n}\right\rangle q^{m}
$$

with the derivative $\mathcal{E}^{\prime}(\tau, n / 2 ; \varphi)$ at $s=n / 2$ of a normalized (and possibly slightly modified; cf. $\S 6$ ) version $\mathcal{E}(\tau, s ; \varphi)$ of the Eisenstein series $E(\tau, s ; \varphi)$. However, it seems a challenge to go beyond the case considered in the present paper and to obtain such results for more general level structures (even for $n=1$ ) and for higher values of $n$, e.g. for $n=2$ (Hilbert-Blumenthal surfaces) or $n=3$ (Siegel threefolds). Nonetheless, the results of [Kud03a] provide some additional evidence in favor of this picture for general $n$. Further discussion of this picture can be found in [Kud01, Kud02b, Kud03b].

With hindsight, it may be said that the results in [KRY99] support our picture in the case $n=0$. This is the one case which is common to the general picture developed here and the general picture of our papers [Kud97b, KR00b, KR99, KR00a, KRY99, Kud02a]. In this rather degenerate case, the variety $X_{K}$ is zero dimensional, so that the cycles $Z(m ; \varphi)$ on the generic fiber are actually empty and the degree generating function $\phi_{\operatorname{deg}}(\tau ; \varphi)$ is identically zero. On the other hand, the associated Eisenstein series $\mathcal{E}(\tau, s ; \varphi)$ of weight 1 is incoherent, in the sense of [Kud97b, Kud02a], so that $\mathcal{E}(\tau, 0 ; \varphi)=0$ as well. The main result of [KRY99, Theorem 3], may be interpreted as the identity

$$
\phi_{\text {height }}\left(\tau, \varphi_{0}\right)=\mathcal{E}^{\prime}\left(\tau, 0 ; \varphi_{0}\right) .
$$

Here, as in the present paper, $\varphi=\varphi_{0}$ is the characteristic function of a certain standard lattice and $K$ is the maximal open compact subgroup. To make the transition to the result in [KRY99], we have to take into account the following two remarks. First, the arithmetic degree $\widehat{\operatorname{deg}}(\cdot)$ on $\widehat{\mathrm{CH}}^{1}\left(\mathfrak{X}_{K}\right)$ used in [KRY99] may be viewed as

$$
\widehat{\operatorname{deg}}(\mathcal{Z})=\left\langle\mathcal{Z}, \hat{\omega}^{0}\right\rangle \text {. }
$$

Second, in defining the degree generating function in this case, we set

$$
\widehat{\mathcal{Z}}(0, v)=\hat{\omega}+(0, \log v) \in \widehat{\mathrm{CH}}^{1}\left(\mathfrak{X}_{K}\right),
$$

where $\hat{\omega}$ is the Hodge bundle on $\mathfrak{X}$ (the moduli stack of elliptic curves with complex multiplication by $\mathrm{O}_{q}$ for a prime $\left.q \equiv 3 \bmod (4)\right)$, with metric normalized as in (10.16) of the present paper. Indeed, this particular choice of normalization, i.e. the choice of the constant $C$ in (10.16) in general and hence in (0.3), was motivated by the requirement that no ambiguous constant like $\mathbf{c}$ should be needed in (0.28). Specifically, the constant term which occurs in [KRY99] is given by

$$
\begin{aligned}
a_{0}(\phi, v) & =-2 h(k)\left(\frac{1}{2} \log (v)+2 h_{\mathrm{Fal}}(E)-\log (2 \pi)-\frac{1}{2} \log (\pi)+\frac{1}{2} \gamma+2 \log (2 \pi)\right) \\
& =-2 h(k)\left(\frac{1}{2} \log (v)+2 h_{\mathrm{Fal}}(E)+\frac{1}{2} \log (\pi)+\frac{1}{2} \gamma+\log (2)\right) \\
& =-2 h(k)\left(\frac{1}{2} \log (v)+2 h_{\mathrm{Fal}}^{*}(E)\right) .
\end{aligned}
$$




\section{Derivatives of Eisenstein series And Faltings heights}

Here the quantity $h_{\mathrm{Fal}}(E)-\frac{1}{2} \log (2 \pi)$ is the Faltings height in the normalization of Colmez [Col93], which was used in (0.16) of [KRY99] (cf. Proposition 10.10 below). We found it particularly striking that the normalization of the metric on the Hodge bundle which eliminates any garbage constant in the case $n=0$ also gives a precise match in the positive Fourier coefficients in our Shimura curve case $(n=1)$. Of course, this is perhaps not so surprising, given the fact that the cycles in the case of the signature $(n, 2)$ are themselves (weighted) combinations of Shimura varieties of the same type for the signature $(n-1,2)$. Thus a main term in the arithmetic degrees which occur in the positive Fourier coefficients of the height generating function for the signature $(n, 2)$ case is the 'arithmetic volume' occurring in the constant term for the $(n-1,2)$ case. This 'explains' the relation between the present paper and the results of [KRY99]. It should not be difficult to verify that the positive Fourier coefficients of the derivatives of Eisenstein series of weight $n / 2+1$ at $s=n / 2$ are related to the constant terms of the derivatives of those of weight $(n-1) / 2+1$ at $s=(n-1) / 2$ in a similar way.

In a similar vein, we remark that the height generating function which, according to our picture above, is related to the derivative of Eisenstein series on $\mathrm{SL}_{2}=\mathrm{Sp}_{1}$ of weight $n / 2+1$ at $s=n / 2$, is connected with the singular Fourier coefficients of the derivative of Eisenstein series of genus 2, i.e. on $\mathrm{Sp}_{2}$, of weight $n / 2+1$ at $s=(n-1) / 2$. In fact, this is how we arrived at the height generating function considered in this paper. We hope to elaborate on this point in a future paper.

\section{Part I. Arithmetic geometry}

\section{The moduli stack $\mathcal{M}$}

Let $B$ be an indefinite quaternion algebra over $\mathbb{Q}$. We fix a maximal order $O_{B}$ in $B$, and we let $D(B)$ be the product of the primes $p$ at which $B_{p}$ is a division algebra. For the moment, we allow the case $B=M_{2}(\mathbb{Q})$ and $O_{B}=M_{2}(\mathbb{Z})$, where $D(B)=1$.

We denote by $\mathcal{M}$ the stack over Spec $\mathbb{Z}$ representing the following moduli problem. The moduli problem associates to a scheme $S$ the category $\mathcal{M}(S)$ whose objects are pairs $(A, \iota)$, where $A$ is an abelian scheme over $S$ and

$$
\iota: O_{B} \longrightarrow \operatorname{End}_{S}(A)
$$

is a homomorphism such that, for $a \in O_{B}$,

$$
\operatorname{det}(\iota(a) ; \operatorname{Lie}(A))=\operatorname{Nm}^{o}(a) .
$$

Here $\mathrm{Nm}^{o}$ is the reduced norm on $B$ and, as usual [Kot92, RZ96], the identity (1.1) is meant as an identity of polynomial functions on $S$. All morphisms in this category are isomorphisms.

Proposition 1.1. $\mathcal{M}$ is an algebraic stack in the sense of Deligne-Mumford. Furthermore, $\mathcal{M}$ is proper over Spec $\mathbb{Z}$ if $B$ is a division algebra. The restriction of $\mathcal{M}$ to $\operatorname{Spec} \mathbb{Z}\left[D(B)^{-1}\right]$ is smooth of relative dimension 1. Finally, if $p \mid D(B)$, then $\mathcal{M} \times_{\text {Spec } \mathbb{Z}}$ Spec $\mathbb{Z}_{p}$ has semi-stable reduction.

\section{Uniformization}

Let $H=B^{\times}$, considered as an algebraic group over $\mathbb{Q}$. Let

$$
D=\operatorname{Hom}_{\mathbb{R}}\left(\mathbb{C}, B_{\mathbb{R}}\right)
$$

be the set of homomorphisms of $\mathbb{R}$-algebras, taking the unit element to the unit element, with the natural conjugation action of $H(\mathbb{R})$. This action is transitive. We fix an isomorphism $B_{\mathbb{R}} \simeq M_{2}(\mathbb{R})$, 


\section{S. S. Kudla, M. RAPOpORT And T. YANG}

so that $H(\mathbb{R}) \simeq G L_{2}(\mathbb{R})$, and a compatible isomorphism $D \simeq \mathbb{C} \backslash \mathbb{R}$, the union of the upper and lower half planes. Also, let $K=\hat{O}_{B}^{\times} \subset H\left(\mathbb{A}_{f}\right)$ be the compact open subgroup determined by $O_{B}$, where $\hat{O}_{B}=O_{B} \otimes_{\mathbb{Z}} \hat{\mathbb{Z}}$. Then we have, as usual, an isomorphism of Deligne-Mumford stacks over $\mathbb{C}$,

$$
\mathcal{M} \times_{\operatorname{Spec} \mathbb{Z}} \operatorname{Spec} \mathbb{C}=\left[H(\mathbb{Q}) \backslash D \times H\left(\mathbb{A}_{f}\right) / K\right],
$$

where the right-hand side is to be understood in the sense of stacks [DM69, p. 99]. The stack on the right-hand side may be written in a simpler way using the fact that $H\left(\mathbb{A}_{f}\right)=H(\mathbb{Q}) K$. Let

$$
\Gamma=H(\mathbb{Q}) \cap K=O_{B}^{\times} .
$$

Then

$$
\mathcal{M} \times \text { Spec } \mathbb{Z} \operatorname{Spec} \mathbb{C}=[\Gamma \backslash D] .
$$

Note that $\Gamma$ acts on $D$ through its image $\bar{\Gamma}=\Gamma /\{ \pm 1\}$ in $\mathrm{PGL}_{2}(\mathbb{R})$, with finite stabilizer groups. Instead of considering $[\Gamma \backslash D]$ as an algebraic stack, it is more traditional to view this quotient as an orbifold [Hai00]. Intuitively speaking, this means that the quotient of $D$ by the action of $\Gamma$ is not carried out, but, rather, all information obtained from the action of $\Gamma$ on $D$ is stored. As a particular instance, consider the hyperbolic volume form $\mu$ on $D$, normalized as

$$
\mu=\frac{1}{2 \pi} y^{-2} d x \wedge d y
$$

in standard coordinates on $\mathbb{C} \backslash \mathbb{R}$. Since this volume form is $\Gamma$-invariant, it induces a volume form on the orbifold $[\Gamma \backslash D]=\mathcal{M}(\mathbb{C})$. The volume of the orbifold $\mathcal{M}(\mathbb{C})$ is given by

$$
\operatorname{vol}(\mathcal{M}(\mathbb{C}))=\int_{[\Gamma \backslash D]} \mu=\frac{1}{2} \int_{\Gamma \backslash D} \mu,
$$

where the extra factor of $\frac{1}{2}$ in the second expression is due to the fact that the stabilizer in $\Gamma$ of a generic point of $D$, has order 2. Explicitly, we have [Eic56]

$$
\operatorname{vol}(\mathcal{M}(\mathbb{C}))=\frac{1}{12} \prod_{p \mid D(B)}(p-1)=-\zeta_{D(B)}(-1),
$$

where

$$
\zeta_{D}(s)=\zeta(s) \prod_{p \mid D}\left(1-p^{-s}\right)
$$

We now turn to $p$-adic uniformization [Dri77, BC91, KR00a]. We fix a prime $p \mid D(B)$. Let $B^{\prime}$ be the definite quaternion algebra over $\mathbb{Q}$ whose invariants agree with those of $B$ at all primes $\ell \neq p, \infty$. Let $H^{\prime}=B^{\prime \times}$ considered as an algebraic group over $\mathbb{Q}$. We fix identifications $H^{\prime}\left(\mathbb{A}_{f}^{p}\right) \simeq H\left(\mathbb{A}_{f}^{p}\right)$ and $H^{\prime}\left(\mathbb{Q}_{p}\right)=G L_{2}\left(\mathbb{Q}_{p}\right)$. Let $\hat{\Omega}^{2}$ be the Deligne-Drinfeld formal scheme relative to $G L_{2}\left(\mathbb{Q}_{p}\right)$. Then

$$
\mathcal{M} \times{ }_{\operatorname{Spec}} \mathbb{Z} \operatorname{Spec} W\left(\overline{\mathbb{F}}_{p}\right) \simeq\left[H^{\prime}(\mathbb{Q}) \backslash\left(\hat{\Omega}^{2} \times_{\operatorname{Spf}} \mathbb{Z}_{p} \operatorname{Spf} W\left(\overline{\mathbb{F}}_{p}\right)\right) \times \mathbb{Z} \times H^{\prime}\left(\mathbb{A}_{f}^{p}\right) / K^{\prime p}\right] .
$$

Here $K^{\prime p}$ corresponds to $\left(O_{B} \otimes \hat{\mathbb{Z}}^{p}\right)^{\times}$under the identification of $H\left(\mathbb{A}_{f}^{p}\right)$ with $H^{\prime}\left(\mathbb{A}_{f}^{p}\right)$, and $g \in H^{\prime}(\mathbb{Q})$ acts on the $\mathbb{Z}$ factor by shifting by $\operatorname{ord}_{p}(\operatorname{det}(g))$. Again, this formula can be simplified since $H^{\prime}(\mathbb{Q}) K_{f}^{p}$ maps surjectively onto $\mathbb{Z} \times H^{\prime}\left(\mathbb{A}_{f}^{p}\right)$. Let

$$
H^{\prime}(\mathbb{Q})^{1}=\left\{g \in H^{\prime}(\mathbb{Q}) \mid \operatorname{ord}_{p}(\operatorname{det}(g))=0\right\} .
$$

Put $\Gamma^{\prime}=H^{\prime}(\mathbb{Q})^{1} \cap K_{f}^{p}$. Then

$$
\mathcal{M} \times_{\operatorname{Spec} \mathbb{Z}} \operatorname{Spec} W\left(\overline{\mathbb{F}}_{p}\right) \simeq\left[\Gamma^{\prime} \backslash \hat{\Omega}^{2} \times_{\operatorname{Spec}} \mathbb{Z}_{p} \operatorname{Spec} W\left(\overline{\mathbb{F}}_{p}\right)\right],
$$

where, again the right-hand side is considered as (the algebraization of) a formal Deligne-Mumford stack. The group $\Gamma^{\prime}$ acts through $\bar{\Gamma}^{\prime}=\Gamma^{\prime} /\{ \pm 1\} \subset \mathrm{PGL}_{2}\left(\mathbb{Q}_{p}\right)$ with finite stabilizer groups. 


\section{DeRivatives of Eisenstein SERIES AND FALtings Heights}

\section{The Hodge bundle}

We denote the universal abelian scheme over $\mathcal{M}$ by $(\mathcal{A}, \iota)$ and its zero section by $\epsilon: \mathcal{M} \rightarrow \mathcal{A}$. The Hodge line bundle on $\mathcal{M}$ is the following line bundle,

$$
\omega=\epsilon^{*}\left(\Omega_{\mathcal{A} / \mathcal{M}}^{2}\right)=\wedge^{2} \operatorname{Lie}(\mathcal{A} / \mathcal{M})^{*} .
$$

For convenience, we will refer to $\omega$ as the Hodge bundle.

Remark 3.1. Assume that $\left(B, O_{B}\right)=\left(M_{2}(\mathbb{Q}), M_{2}(\mathbb{Z})\right)$. In this case, $\mathcal{M}$ may be identified with the moduli stack of elliptic curves and the universal object $\mathcal{A}$ with $\left(\mathcal{E}^{2}, \iota_{0}\right)$, where $\mathcal{E}$ is the universal elliptic curve and $\iota_{0}: M_{2}(\mathbb{Z}) \rightarrow \operatorname{End}\left(\mathcal{E}^{2}\right)=M_{2}(\operatorname{End}(\mathcal{E}))$ is the natural embedding. In this case, $\operatorname{Lie}(\mathcal{A} / \mathcal{M})=\operatorname{Lie}(\mathcal{E} / \mathcal{M})^{\oplus 2}$ and hence

$$
\omega=\omega_{\mathcal{E} / \mathcal{M}}^{\otimes 2}
$$

Here $\omega_{\mathcal{E} / \mathcal{M}}=\operatorname{Lie}(\mathcal{E} / \mathcal{M})^{*}$. Recall [DR73, VI.4.5], that $\omega_{\mathcal{E} / \mathcal{M}}^{\otimes 2}$ can be identified with the module of relative differentials of $\mathcal{M} / \operatorname{Spec} \mathbb{Z}$. The following proposition generalizes this fact.

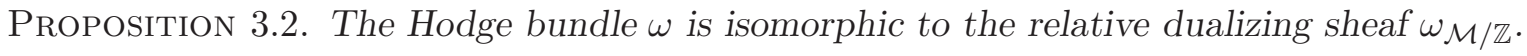

Proof. Since the fibers of $\mathcal{M}$ over Spec $\mathbb{Z}$ are Gorenstein, $\omega_{\mathcal{M} / \mathbb{Z}}$ is an invertible sheaf. Since $\mathcal{M}$ is regular of dimension 2 , it suffices to show that the restriction of $\omega$ to the smooth locus $\mathcal{M}^{\text {smooth }}$ is isomorphic to the restriction of $\omega_{\mathcal{M} / \mathbb{Z}}$ to $\mathcal{M}^{\text {smooth }}$, i.e. to the sheaf of relative differentials $\Omega_{\mathcal{M}}^{1}$ smooth $/ \mathbb{Z}$.

By deformation theory we have a canonical identification

$$
\Omega_{\mathcal{M}^{\text {smooth } / \mathbb{Z}}}^{1}=\operatorname{Hom}_{O_{B}}\left(\operatorname{Lie} \mathcal{A},\left(\operatorname{Lie}(\hat{\mathcal{A}})^{*}\right),\right.
$$

where $(\mathcal{A}, \iota)$ is, as before, the universal object over $\mathcal{M}$ and where $\hat{\mathcal{A}}$ denotes the dual abelian variety.

This formula shows that it suffices to check the claimed equality after passing to the completion $\mathbb{Z}_{p}$ for each prime $p$. For any prime $p \nmid D(B)$ our identification problem reduces to the situation considered in Remark 3.1. Hence, all we need to do is to extend to $\mathcal{M}^{\text {smooth }}$ the isomorphism between $\omega$ and $\Omega_{\mathcal{M} / \text { Spec }}^{1}$ over $\mathcal{M}\left[D(B)^{-1}\right]$. Fix a prime number $p \mid D(B)$. Denote by $R$ the involution on $O_{B}$

$$
\alpha \longmapsto \alpha^{R}=\delta \alpha^{\iota} \delta^{-1},
$$

where $\delta \in O_{B}$ satisfies $\delta^{2}=-D(B)$ and where $\alpha \mapsto \alpha^{\iota}$ is the main involution on $B$. After choosing a $p$-principal polarization on $\mathcal{A}$ whose Rosati involution induces the involution $R$ on $O_{B}$, we may identify $(\operatorname{Lie} \hat{\mathcal{A}})^{*}$ with $(\operatorname{Lie} \mathcal{A})^{*}$, in such a way that $\alpha \in O_{B}$ acts on $(\operatorname{Lie} \mathcal{A})^{*}$ as $\left(\alpha^{R}\right)^{*}$. We write

$$
O_{B_{p}}=\mathbb{Z}_{p^{2}}[\Pi] /\left(\Pi^{2}=p, \Pi a=a^{\sigma} \Pi, \forall a \in \mathbb{Z}_{p^{2}}\right) .
$$

Here $\mathbb{Z}_{p^{2}}$ is the ring of integers in the unramified quadratic extension of $\mathbb{Z}_{p}$. We may assume that the restriction of $R$ to $\mathbb{Z}_{p^{2}}$ is trivial and that $\Pi^{R}=-\Pi$. After extending scalars from $\mathbb{Z}$ to $\mathbb{Z}_{p^{2}}$, we have the eigenspace decomposition of $\operatorname{Lie} \mathcal{A}$ as

$$
\text { Lie } \mathcal{A}=\mathcal{L}_{0} \oplus \mathcal{L}_{1}
$$

such that the action of $\Pi$ on Lie $\mathcal{A}$ is of degree 1 with respect to this $\mathbb{Z} / 2$-grading. The condition (1.1) ensures that $\mathcal{L}_{0}$ and $\mathcal{L}_{1}$ are both line bundles. It now follows, via (3.3) and (3.6), that the local sections of $\Omega_{\mathcal{M} / \mathbb{Z}}^{1}$ are given by local homomorphisms $\varphi_{i}: \mathcal{L}_{i} \rightarrow \mathcal{L}_{i}^{*}(i=0,1)$ forming a 
commutative diagram

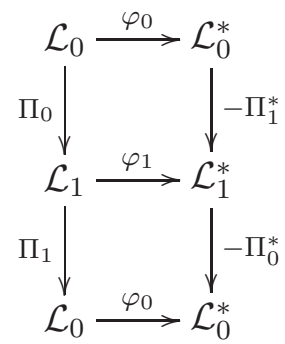

The pair $\left(\varphi_{0}, \varphi_{1}\right)$ therefore defines an injective homomorphism

$$
\alpha: \Omega_{\mathcal{M} / \mathbb{Z}}^{1} \longrightarrow \mathcal{L}_{0}^{\otimes(-2)} \oplus \mathcal{L}_{1}^{\otimes(-2)} \subset(\operatorname{Lie} \mathcal{A})^{*} \otimes(\operatorname{Lie} \mathcal{A}) .
$$

On the generic fiber, $\mathcal{L}_{0}^{\otimes(-2)}$ and $\mathcal{L}_{1}^{\otimes(-2)}$ both coincide with $\omega$ and $\alpha$ induces an isomorphism of $\Omega_{\mathcal{M} / \mathbb{Z}}^{1}$ with the diagonal. On the smooth locus, either $\Pi_{0}$ or $\Pi_{1}$ is an isomorphism locally around any given point. Assume for instance that $\Pi_{0}$ is an isomorphism. Then $\varphi_{0}$ determines $\varphi_{1}$ by the commutativity of the upper square in (3.7),

$$
\varphi_{1}=\left(-\Pi_{1}^{*}\right) \circ \varphi_{0} \circ \Pi_{0}^{-1} .
$$

But then the lower square also commutes. Since $\Pi_{0}$ is an isomorphism, it suffices to check this after premultiplying $\varphi_{1}$ with $\Pi_{0}$. However,

$$
\left(-\Pi_{0}^{*}\right) \circ \varphi_{1} \circ \Pi_{0}=\left(-\Pi_{0}^{*}\right) \circ\left(-\Pi_{1}^{*}\right) \circ \varphi_{0}=\varphi_{0} \circ \Pi_{1} \circ \Pi_{0} .
$$

It follows that on the open sublocus of $\mathcal{M}^{\text {smooth }}$ where $\Pi_{0}$ is an isomorphism, the first projection applied to (3.8) induces an isomorphism between $\Omega_{\mathcal{M} / \mathbb{Z}}^{1}$ and $\mathcal{L}_{0}^{\otimes(-2)}$. On the other hand, on this open sublocus, $\omega$ can be identified with $\mathcal{L}_{0}^{\otimes(-2)}$, which proves the claim.

By base change to $\mathbb{C}$, the Hodge bundle induces a line bundle $\omega_{\mathbb{C}}$ on $\mathcal{M}_{\mathbb{C}}=[\Gamma \backslash D]$. In the orbifold picture, we may view $\omega_{\mathbb{C}}$ as being given by a descent datum with respect to the action of $\Gamma$ on the pullback of $\omega_{\mathbb{C}}$ to $D$. At a point $z$ of $\mathcal{M}_{\mathbb{C}}$, a section $\alpha$ of $\omega_{\mathbb{C}}$ corresponds a holomorphic 2 form on $\mathcal{A}_{z}$ and so there is a natural norm [Bos99] on $\omega_{\mathbb{C}}$ given by

$$
\left\|\alpha_{z}\right\|_{\text {nat }}^{2}=\left|\left(\frac{i}{2 \pi}\right)^{2} \int_{\mathcal{A}_{z}(\mathbb{C})} \alpha \wedge \bar{\alpha}\right|
$$

Equivalently, $\omega_{\mathbb{C}}$ is given by the automorphy factor $(c z+d)^{2}$, i.e. by the action of $\Gamma$ on $D \times \mathbb{C}$ defined by

$$
\gamma=\left(\begin{array}{ll}
a & b \\
c & d
\end{array}\right):(z, \zeta) \mapsto\left(\gamma(z),(c z+d)^{2} \zeta\right) .
$$

More precisely, for $z \in D \simeq \mathbb{C} \backslash \mathbb{R}$, we have an isomorphism

$$
B_{\mathbb{R}} \simeq M_{2}(\mathbb{R}) \stackrel{\sim}{\longrightarrow} \mathbb{C}^{2}, \quad u \mapsto u \cdot\left(\begin{array}{l}
z \\
1
\end{array}\right)=\left(\begin{array}{l}
w_{1} \\
w_{2}
\end{array}\right),
$$

and the corresponding abelian variety $\mathcal{A}_{z}$ has $\mathcal{A}_{z}(\mathbb{C})=\mathbb{C}^{2} / \Lambda_{z}$, where $\Lambda_{z}$ is the image of $O_{B}$ in $\mathbb{C}^{2}$. The pullback of $\omega_{\mathbb{C}}$ to $D$ is trivialized via the section $\alpha=d w_{1} \wedge d w_{2}$. The Petersson norm, $\|\cdot\|_{\text {Pet }}$ on the bundle of modular forms of weight 2 , is defined by the $\Gamma$-invariant norm on the trivial line bundle $D \times \mathbb{C}$ given by

$$
\|(z, \zeta)\|_{\text {Pet }}^{2}=|\zeta|^{2}(4 \pi \operatorname{Im}(z))^{2} .
$$

If $f$ is such a modular form, then we identify $f$ with the section [CF90, pp. 141-2],

$$
\alpha(f)=f(z)\left(2 \pi i d w_{1} \wedge 2 \pi i d w_{2}\right)=-4 \pi^{2} f(z) \alpha .
$$




\section{Derivatives of Eisenstein series AND FAltings Heights}

Lemma 3.3. The two norms on $\omega_{\mathbb{C}}$ are related by

$$
\|\cdot\|_{\text {nat }}^{2}=D(B)\|\cdot\|_{\text {Pet }}^{2} \text {. }
$$

Proof. The pullback to $M_{2}(\mathbb{R})$ of the form $\alpha \wedge \bar{\alpha}=d w_{1} \wedge d w_{2} \wedge d \bar{w}_{1} \wedge d \bar{w}_{2}$ above is $4 \operatorname{Im}(z)^{2}$ times the standard volume form, and so, via (3.11),

$$
\|\alpha\|_{\text {nat }}^{2}=\frac{1}{4 \pi^{2}} \cdot 4 \operatorname{Im}(z)^{2} \operatorname{vol}\left(M_{2}(\mathbb{R}) / O_{B}\right)
$$

Then

$$
\|\alpha(f)\|_{\text {nat }}^{2}=16 \pi^{4}|f(z)|^{2} \cdot \frac{D(B)}{\pi^{2}} \operatorname{Im}(z)^{2} .
$$

Definition 3.4. The metrized Hodge bundle $\hat{\omega}$ is $\omega$ equipped with the metric

$$
\|\cdot\|=e^{-C}\|\cdot\|_{\text {nat }},
$$

where $C=\frac{1}{2}(\log (4 \pi)+\gamma)$. Here $\gamma$ is Euler's constant.

The motivation for this normalization is explained in the introduction.

The Chern form $c_{1}(\hat{\omega})$ for this metric is then

$$
c_{1}(\hat{\omega})=-d d^{c} \log \|\alpha\|^{2}=\mu
$$

with $\mu$ as in $(2.5)$, and so

$$
\operatorname{deg}(\hat{\omega})=\int_{[\Gamma \backslash D]} c_{1}(\hat{\omega})=\operatorname{vol}(\mathcal{M}(\mathbb{C})) .
$$

\section{The arithmetic Picard group and the arithmetic Chow group}

From now on, we assume that $D(B)>1$, so that $B$ is a division algebra and $\mathcal{M}$ is proper over Spec $\mathbb{Z}$. If we had imposed a sufficient level structure, then $\mathcal{M}$ would be an arithmetic surface over Spec $\mathbb{Z}$ [GS90, Bos99]. Then the Chow groups (tensored with $\mathbb{Q}) \mathrm{CH}^{r}(\mathcal{M})$ and arithmetic Chow groups $\widehat{\mathrm{CH}}^{r}(\mathcal{M})$ would be defined, with $\widehat{\mathrm{CH}}^{1}(\mathcal{M}) \simeq \widehat{\mathrm{Pic}}(\mathcal{M})$, the group of isomorphism classes of metrized line bundles, and these would be equipped with a height pairing

$$
\langle\cdot, \cdot\rangle: \widehat{\mathrm{CH}}^{1}(\mathcal{M}) \times \widehat{\mathrm{CH}}^{1}(\mathcal{M}) \longrightarrow \widehat{\mathrm{CH}}^{2}(\mathcal{M})
$$

and the arithmetic degree

$$
\widehat{\operatorname{deg}}: \widehat{\mathrm{CH}}^{2}(\mathcal{M}) \longrightarrow \mathbb{C} .
$$

In this section, we explain how to carry over (parts of) this formalism to our DM-stack $\mathcal{M}$.

We begin with $\widehat{\operatorname{Pic}}(\mathcal{M})$. There are two ways to define the concept of a metrized line bundle on $\mathcal{M}$. First, one can define such an object to be a rule which associates, functorially, to any $S$-valued point $S \rightarrow \mathcal{M}$ of $\mathcal{M}$, a line bundle $\mathcal{L}_{S}$ on $S$ equipped with a $C^{\infty}$-metric on the line bundle $\mathcal{L}_{S, \mathbb{C}}$ on $S \times_{\text {Spec } \mathbb{Z}}$ Spec $\mathbb{C}$. Second, one can define a metrized line bundle on $\mathcal{M}$ to be an invertible sheaf on $\mathcal{M}$ together with a $\Gamma$-invariant metric on the pullback of $\mathcal{L}_{\mathbb{C}}$ to $D$ under the identification of $\mathcal{M}_{\mathbb{C}}$ with the orbifold $[\Gamma \backslash D]$. These definitions are equivalent. As usual, we denote the set of isomorphism classes of metrized line bundles on $\mathcal{M}$ by $\widehat{\operatorname{Pic}}(\mathcal{M})$. This is an abelian group under the tensor product operation.

Let $\hat{\mathcal{L}}=(\mathcal{L},\|\cdot\|)$ be a metrized line bundle on $\mathcal{M}$. Then $\hat{\mathcal{L}}$ determines a height of a onedimensional irreducible reduced proper DM-stack $\mathcal{Z}$ mapping to $\mathcal{M}$. To define it, we are guided by the heuristic principle that, in a numerical formula, a geometric point $x$ of a stack counts with 


\section{S. S. Kudla, M. Rapoport And T. YAng}

fractional multiplicity $1 /|\operatorname{Aut}(x)|$. Let $\tilde{\mathcal{Z}}$ be the normalization and $\nu: \tilde{\mathcal{Z}} \rightarrow \mathcal{M}$ be the natural morphism.

If $\Gamma\left(\tilde{\mathcal{Z}}, \mathcal{O}_{\tilde{\mathcal{Z}}}\right)=O_{K}$ for a number field $K$, then

$$
h_{\hat{\mathcal{L}}}(\mathcal{Z})=\widehat{\operatorname{deg}} \nu^{*}(\hat{\mathcal{L}}) .
$$

Here the right-hand side is defined by setting, for a meromorphic section $s$ of $\nu^{*}(\mathcal{L})$,

$$
\widehat{\operatorname{deg}} \nu^{*}(\hat{\mathcal{L}})=\sum_{p}\left(\sum_{x \in \tilde{\mathcal{Z}}\left(\overline{\mathbb{F}}_{p}\right)} \frac{\operatorname{ord}_{x}(s)}{|\operatorname{Aut}(x)|}\right) \cdot \log p-\frac{1}{2} \int_{\tilde{\mathcal{Z}}(\mathbb{C})} \log \|s\|^{2} .
$$

Here the integral is defined as

$$
\int_{\tilde{\mathcal{Z}}(\mathbb{C})} \log \|s\|^{2}=\sum_{z \in \tilde{\mathcal{Z}}(\mathbb{C})} \frac{1}{|\operatorname{Aut}(z)|} \cdot \log \|s(z)\|^{2} .
$$

Also, $\operatorname{ord}_{x}(s)$ is defined by noting that the strict henselization $\tilde{\mathcal{O}}_{\tilde{\mathcal{Z}}, x}$ of the local $\operatorname{ring} \mathcal{O}_{\tilde{\mathcal{Z}}, x}$ is a discrete valuation ring. Let us check that the expression (4.4) is independent of the choice of $s$. This comes down to checking, for a function $f \in \mathbb{Q}(\tilde{\mathcal{Z}})^{\times}=K^{\times}$, that

$$
0=\sum_{p}\left(\sum_{x \in \tilde{\mathcal{Z}}\left(\overline{\mathbb{F}}_{p}\right)} \frac{\operatorname{ord}_{x}(f)}{|\operatorname{Aut}(x)|}\right) \cdot \log p-\frac{1}{2} \sum_{\sigma: K \rightarrow \mathbb{C}} \frac{1}{|\operatorname{Aut}(\sigma)|} \cdot \log |\sigma(f)|^{2} .
$$

For $x \in \tilde{\mathcal{Z}}\left(\overline{\mathbb{F}}_{p}\right)$, let $\underline{x}$ be the corresponding geometric point of the coarse moduli scheme $Z=\operatorname{Spec} O_{K}$ of $\tilde{\mathcal{Z}}$. Then

$$
\tilde{\mathcal{O}}_{Z, \underline{x}}=\left(\tilde{\mathcal{O}}_{\tilde{\mathcal{Z}}, x}\right)^{\operatorname{Aut}(x) / \operatorname{Aut}(\bar{\eta})},
$$

where $\bar{\eta}$ is any generic geometric point of $\tilde{\mathcal{Z}}$, and $\tilde{\mathcal{O}}_{\tilde{\mathcal{Z}}, x}$ is a totally ramified extension of degree $|\operatorname{Aut}(x)| /|\operatorname{Aut}(\bar{\eta})|$ of $\tilde{\mathcal{O}}_{Z, \underline{x}}$. Inserting this into (4.6), we obtain for the right-hand side the expression

$$
\frac{1}{|\operatorname{Aut}(\bar{\eta})|}\left(\sum_{p} \sum_{x \in\left(\operatorname{Spec} O_{K}\right)\left(\overline{\mathbb{F}}_{p}\right)} \operatorname{ord}_{x}(f) \cdot \log p-\sum_{\sigma} \log |\sigma(f)|\right)
$$

which is zero by the product formula for $f \in K^{\times}$.

If $\Gamma\left(\tilde{\mathcal{Z}}, \mathcal{O}_{\tilde{\mathcal{Z}}}\right)=\mathbb{F}_{q}$, we put

$$
h_{\hat{\mathcal{L}}}(\mathcal{Z})=\operatorname{deg} \nu^{*}(\mathcal{L}) \cdot \log q=\left(\sum_{x \in \tilde{\mathcal{Z}}\left(\overline{\mathbb{F}}_{p}\right)} \frac{\operatorname{ord}_{x}(s)}{|\operatorname{Aut}(x)|}\right) \cdot \log p,
$$

where $s$ is a meromorphic section of $\nu^{*}(\mathcal{L})$. Here $\operatorname{deg} \nu^{*}(\mathcal{L})$ coincides with the definition given in [DR73, V.4.3].

Next we need to define the (arithmetic) Chow group of $\mathcal{M}$. By a prime divisor on $\mathcal{M}$ we mean an irreducible closed substack $\mathcal{Z}$ of $\mathcal{M}$ which is locally, for the étale topology, a Cartier divisor defined by an irreducible equation. Let $Z^{1}(\mathcal{M})$ be the free abelian group generated by the prime divisors on $\mathcal{M}$. Any rational function $f \in \mathbb{Q}(\mathcal{M})^{\times}$(i.e. a morphism $\mathcal{U} \rightarrow \mathbb{A}^{1}$ defined on a non-empty open substack $\mathcal{U}$ of $\mathcal{M})$ defines a principal divisor

$$
\operatorname{div}(f)=\sum_{\mathcal{Z}} \operatorname{ord}_{\mathcal{Z}}(f) \cdot \mathcal{Z}
$$

where the sum is over the prime divisors $\mathcal{Z}$ of $\mathcal{M}$, and where we note that the strict henselization of the local ring at $\mathcal{Z}, \tilde{\mathcal{O}}_{\mathcal{M}, \mathcal{Z}}$, is a discrete valuation ring. The factor group of $Z^{1}(\mathcal{M})$ by the group of principal divisors is the Chow group $\mathrm{CH}^{1}(\mathcal{M})$ (cf. [Vis89]). 


\section{DeRivatives of Eisenstein SERIES AND FALtings Heights}

Let $\mathcal{Z} \in Z^{1}(\mathcal{M})$. Then the divisor $\mathcal{Z}_{\mathbb{C}}$ of $\mathcal{M}_{\mathbb{C}}=[\Gamma \backslash D]$ is of the form $\mathcal{Z}_{\mathbb{C}}=\left[\Gamma \backslash D_{\mathcal{Z}}\right]$ for a unique $\Gamma$-invariant divisor $D_{\mathcal{Z}}$ of $D$. By a Green's function for $\mathcal{Z}$ we mean a real current $g$ of degree zero on $D$ which is $\Gamma$-invariant and such that

$$
\omega=d d^{c} g+\delta_{D_{\mathcal{Z}}}
$$

is $C^{\infty}$. We denote by $\hat{Z}^{1}(\mathcal{M})$ the group of Arakelov divisors, i.e. of pairs $(\mathcal{Z}, g)$ consisting of a divisor $\mathcal{Z}$ on $\mathcal{M}$ and a Green's function for $\mathcal{Z}$, with componentwise addition. If $f \in \mathbb{Q}(\mathcal{M})^{\times}$, then $f \mid \mathcal{M}_{\mathbb{C}}$ corresponds to a $\Gamma$-invariant meromorphic function $\tilde{f}_{\mathbb{C}}$ on $D$ and we define the associated principal Arakelov divisor

$$
\widehat{\operatorname{div}}(f)=\left(\operatorname{div}(f),-\log \left|\tilde{f}_{\mathbb{C}}\right|^{2}\right) .
$$

The factor group of $\hat{Z}^{1}(\mathcal{M})$ by the group of principal Arakelov divisors is the arithmetic Chow group $\widehat{\mathrm{CH}}^{1}(\mathcal{M})$. The groups $\widehat{\mathrm{CH}}^{1}(\mathcal{M})$ and $\widehat{\mathrm{Pic}}(\mathcal{M})$ are isomorphic. Under this isomorphism, an element $\hat{\mathcal{L}}$ goes to the class of

$$
\left(\sum_{\mathcal{Z}} \operatorname{ord}_{\mathcal{Z}}(s) \mathcal{Z},-\log \|s\|^{2}\right)
$$

where $s$ is a meromorphic section of $\mathcal{L}$. Conversely, if $(\mathcal{Z}, g) \in Z^{1}(\mathcal{M})$, then its preimage under this isomorphism is

$$
(\mathcal{O}(\mathcal{Z}),\|\cdot\|)
$$

where $-\log \|\mathbf{1}\|^{2}=g$, with $\mathbf{1}$ the canonical $\Gamma$-invariant section of the pullback of $\hat{\mathcal{O}}(\mathcal{Z})$ to $D$.

We define a pairing

$$
\langle\cdot, \cdot\rangle: \hat{Z}^{1}(\mathcal{M}) \times \widehat{\operatorname{Pic}}(\mathcal{M}) \longrightarrow \mathbb{C}
$$

by formula (5.11) in Bost [Bos99],

$$
\langle(\mathcal{Z}, g), \hat{\mathcal{L}}\rangle=h_{\hat{\mathcal{L}}}(\mathcal{Z})+\frac{1}{2} \int_{[\Gamma \backslash D]} g \cdot c_{1}(\hat{\mathcal{L}}) .
$$

Here $c_{1}(\hat{\mathcal{L}})$ is the $\Gamma$-invariant form on $D$ defined by the pullback to $D$ of $\hat{\mathcal{L}}$ (analogously to $c_{1}(\hat{\omega})$ in $\S 3$ above). The integral is defined as

$$
\int_{[\Gamma \backslash D]} g \cdot c_{1}(\hat{\mathcal{L}})=\left|\Gamma^{\prime}\right|^{-1} \cdot \int_{\Gamma \backslash D} g \cdot c_{1}(\hat{\mathcal{L}}),
$$

where $\Gamma^{\prime}=\operatorname{ker}(\Gamma \rightarrow \operatorname{Aut}(D))$.

It seems very likely that under the identification $\widehat{\mathrm{CH}}^{1}(\mathcal{M}) \simeq \widehat{\operatorname{Pic}}(\mathcal{M})$, the pairing $(4.15)$ descends to a symmetric bilinear pairing

$$
\langle\cdot, \cdot\rangle: \widehat{\mathrm{CH}}^{1}(\mathcal{M}) \times \widehat{\mathrm{CH}}^{1}(\mathcal{M}) \longrightarrow \mathbb{C},
$$

as is the case for arithmetic surfaces. For ease of expression we will proceed as if this were the case, although we have not checked it. Since all we will actually use is the pairing (4.15), this will cause no harm.

\section{Special cycles and the generating function}

In this section, we will define for each $m \in \mathbb{Z}$ and each $v \in \mathbb{R}_{+}^{\times}$a class

$$
\hat{\mathcal{Z}}(m, v)=(\mathcal{Z}(m), \Xi(m, v)) \in \widehat{\mathrm{CH}}^{1}(\mathcal{M}) .
$$

We first assume that $m>0$. Then we consider the DM-stack $\mathcal{Z}(m)$ classifying triples $(A, \iota, x)$ where $(A, \iota)$ is an object of $\mathcal{M}$ and where $x$ is a special endomorphism [Kud97b, KR00a], with 


\section{S. S. Kudla, M. Rapoport And T. YAnG}

$x^{2}=-m$, i.e.

$$
x \in \operatorname{End}(A, \iota), \quad \operatorname{tr}^{o}(x)=0, \quad x^{2}=-m .
$$

Then $\mathcal{Z}(m)$ maps to $\mathcal{M}$ by a finite unramified morphism. Furthermore, $\mathcal{Z}(m)$ is purely one dimensional, except in the following cases (see [KR00a] and Appendix A),

$$
\exists p \mid D(B), \quad p \neq 2, \quad \text { such that } m \in \mathbb{Z}_{p}^{\times, 2} .
$$

In the cases covered by $(5.3)$, we set $\hat{\mathcal{Z}}(m, v)=0$. In all other cases, we define a Green's function for the unramified morphism $\mathcal{Z}(m) \rightarrow \mathcal{M}$, in the sense of $\S 4$, as follows [Kud97b]. Let

$$
V=\left\{x \in B \mid \operatorname{tr}^{\circ}(x)=0\right\}
$$

with quadratic form $Q(x)=-x^{2}=N^{o}(x)$ given by the restriction of the reduced norm and with associated inner product $(x, y)=\operatorname{tr}^{o}\left(x y^{\iota}\right)$. Note that the signature of $V(\mathbb{R})$ is $(1,2)$. As in [Kud97b], we can identify $D$ with the space of oriented negative 2-planes in $V(\mathbb{R})$. For $x \in V(\mathbb{R})$ and $z \in D$, let $\operatorname{pr}_{z}(x)$ be the projection of $x$ to $z$ and let

$$
R(x, z)=-\left(\operatorname{pr}_{z}(x), \operatorname{pr}_{z}(x)\right) \geqslant 0 .
$$

This quantity vanishes precisely when $\operatorname{pr}_{z}(x)=0$, i.e. when $z \in D_{x}$ where

$$
D_{x}=\{z \in D \mid(x, z)=0\} .
$$

Let $L=V(\mathbb{Q}) \cap O_{B}$, and let

$$
L(m)=\{x \in L \mid Q(x)=m\} .
$$

Then, for $m \in \mathbb{Z}_{\neq 0}$ and $v \in \mathbb{R}_{+}^{\times}$, let

$$
\Xi(m, v)=\sum_{x \in L(m)} \xi\left(v^{\frac{1}{2}} x, z\right)
$$

where

for the exponential integral

$$
\xi(x, z)=-\operatorname{Ei}(-2 \pi R(x, z))
$$

$$
-\operatorname{Ei}(-t)=\int_{1}^{\infty} e^{-t r} r^{-1} d r .
$$

The properties of this function are described in [Kud97b, $\S 11]$. For $m>0, \Xi(m, v)$ is a $\Gamma$-invariant Green's function for the divisor

$$
D_{\mathcal{Z}(m)}:=\coprod_{x \in L(m)} D_{x}
$$

in $D$.

When $m<0, \Xi(m, v)$ is a smooth $\Gamma$-invariant function on $D$. Therefore,

$$
\hat{\mathcal{Z}}(m, v)=(0, \Xi(m, v)), \quad m<0,
$$

again defines an element of $\widehat{\mathrm{CH}}^{1}(\mathcal{M})$.

For $m=0$, the definition of $\hat{\mathcal{Z}}(0, v)$ is more speculative. Using the canonical map from $\widehat{\operatorname{Pic}}(\mathcal{M})$ to $\widehat{\mathrm{CH}}^{1}(\mathcal{M})$, we let

$$
\hat{\mathcal{Z}}(0, v)=-\hat{\omega}-(0, \log v)+(0, \mathbf{c}),
$$

where the constant $\mathbf{c}$ has the value given in (0.15).

We have now defined elements $\hat{\mathcal{Z}}(m, v) \in \widehat{\mathrm{CH}}^{1}(\mathcal{M})$ for all $m \in \mathbb{Z}$ and $v \in \mathbb{R}_{+}^{\times}$. We define the following two generating series, which are formal Laurent series in a parameter $q$. Later we take $q=e(\tau)=e^{2 \pi i \tau}$, where $\tau=u+i v \in \mathfrak{H}$. 


\section{DeRivatives of Eisenstein SERIES AND FALtings Heights}

The first generating function involves only the orbifold $\mathcal{M}(\mathbb{C})=[\Gamma \backslash D]$. Let

$$
\omega(m, v)=d d^{c} \Xi(m, v)+\delta_{D_{\mathcal{Z}(m)}}
$$

be the right-hand side of the Green's equation for $\Xi(m, v)$. Then let

$$
\operatorname{deg}(\hat{\mathcal{Z}}(m, v))=\int_{[\Gamma \backslash D]} \omega(m, v) .
$$

If $m>0$, then $\operatorname{deg}(\hat{\mathcal{Z}}(m, v))$ is just the usual degree of the 0 -cycle $\mathcal{Z}(m)_{\mathbb{C}}$ (in the stack sense). If $m<0$, then $\operatorname{deg}(\hat{\mathcal{Z}}(m, v))=0$, since $\Xi(m, v)$ is smooth in this case so that $\omega(m, v)$ is exact. For $m=0$, we take $\omega(0, v)$ to be the Chern form of $-\hat{\omega}$, i.e. $-\mu$, and hence

$$
\operatorname{deg}(\hat{\mathcal{Z}}(0, v)):=\int_{[\Gamma \backslash D]} \omega(0, v)=-\operatorname{vol}(\mathcal{M}(\mathbb{C})) .
$$

The generating function for degrees is then

$$
\begin{aligned}
\phi_{\operatorname{deg}}(\tau) & :=\sum_{m} \operatorname{deg}(\hat{\mathcal{Z}}(m, v)) q^{m} \\
& =-\operatorname{vol}(\mathcal{M}(\mathbb{C}))+\sum_{m>0} \operatorname{deg}\left(\mathcal{Z}(m)_{\mathbb{C}}\right) q^{m} .
\end{aligned}
$$

For the second generating function, we use the height pairing (4.15) of our cycles with the class $\hat{\omega} \in \widehat{\operatorname{Pic}}(\mathcal{M})$, and let

$$
\phi_{\text {height }}(\tau)=\sum_{m}\langle\hat{\mathcal{Z}}(m, v), \hat{\omega}\rangle q^{m} .
$$

For the moment, we regard $\phi_{\operatorname{deg}}(\tau)$ (respectively $\left.\phi_{\text {height }}(\tau)\right)$ as a formal generating series, but our main theorem will identify it as a bona fide holomorphic (respectively non-holomorphic) function of the variable $\tau$ by identifying it with the Fourier expansion of a special value of an Eisenstein series (respectively of the derivative of an Eisenstein series).

\section{Part II. Eisenstein series}

\section{Eisenstein series of weight $\frac{3}{2}$}

In this section, we introduce the Eisenstein series of half-integral weight which will be connected with the arithmetic geometry discussed in Part I. A more general discussion of such series from an adèlic point of view can be found in [Kud97b]. The series we consider are, of course, rather familiar from a classical point of view, and an expression for them in this language will emerge in $\S \S 8$ and 15 below. Thus, one purpose of the present section is to explain how such classical series are associated to indefinite quaternion algebras in a natural way, via the Weil representation. A second advantage of the adèlic viewpoint is that it allows one to assemble the Fourier coefficients out of local quantities. This construction shows in a very clear way the dependence of these coefficients and, more importantly, of their derivatives on the choice of local data.

Let $G_{\mathbb{A}}^{\prime}$ be the metaplectic extension of $\mathrm{Sp}_{1}(\mathbb{A})=\mathrm{SL}_{2}(\mathbb{A})$ by $\mathbb{C}^{1}$ and let $P_{\mathbb{A}}^{\prime}$ be the preimage of the subgroup $N(\mathbb{A}) M(\mathbb{A})$ of $\mathrm{SL}_{2}(\mathbb{A})$ where

$$
N(\mathbb{A})=\left\{n(b)=\left(\begin{array}{ll}
1 & b \\
0 & 1
\end{array}\right) \mid b \in \mathbb{A}\right\}
$$

and

$$
M(\mathbb{A})=\left\{m(a)=\left(\begin{array}{cc}
a & 0 \\
0 & a^{-1}
\end{array}\right) \mid a \in \mathbb{A}^{\times}\right\}
$$




\section{S. S. Kudla, M. Rapoport And T. YAnG}

As in [Wal80], we have an identification $G_{\mathbb{A}}^{\prime} \simeq \mathrm{SL}_{2}(\mathbb{A}) \times \mathbb{C}^{1}$ where the multiplication on the right-hand side is given by $\left[g_{1}, z_{1}\right]\left[g_{2}, z_{2}\right]=\left[g_{1} g_{2}, c\left(g_{1}, g_{2}\right) z_{1} z_{2}\right]$ with cocycle $c\left(g_{1}, g_{2}\right)$ as in [Wal80] or [Gel76]. Let $G_{\mathbb{Q}}^{\prime}=\mathrm{SL}_{2}(\mathbb{Q})$, identified with a subgroup of $G_{\mathbb{A}}^{\prime}$ via the canonical splitting homomorphism $G_{\mathbb{Q}}^{\prime} \rightarrow$ $G_{\mathbb{A}}^{\prime}$, and let $P_{\mathbb{Q}}^{\prime}=P_{\mathbb{A}}^{\prime} \cap G_{\mathbb{Q}}^{\prime}$. An idèle character $\chi$ of $\mathbb{Q}^{\times} \backslash \mathbb{A}^{\times}$determines a character $\chi^{\psi}$ of $P_{\mathbb{Q}}^{\prime} \backslash P_{\mathbb{A}}^{\prime}$ via

$$
\chi^{\psi}([n(b) m(a), z])=z \chi(a) \gamma(a, \psi)^{-1},
$$

where $\psi$ is our fixed additive character of $\mathbb{Q} \backslash \mathbb{A}$ and $\gamma(a, \psi)$ is the Weil index ([Wei64] or [Rao93, Appendix]). For $s \in \mathbb{C}$, let

$$
I(s, \chi)=\operatorname{Ind}_{P_{\mathbb{A}}^{\prime}}^{G_{A}^{\prime}} \chi^{\psi}|\cdot|^{s}
$$

be the principal series representation of $G_{\mathbb{A}}^{\prime}$ determined by $\chi^{\psi}$. A section $\Phi(s) \in I(s, \chi)$ is thus a smooth function on $G_{\mathbb{A}}^{\prime}$ such that

$$
\Phi\left(p^{\prime} g^{\prime}, s\right)=\chi^{\psi}\left(p^{\prime}\right)|a|^{s+1} \Phi\left(g^{\prime}, s\right),
$$

where $p^{\prime}=[n(b) m(a), z]$. Such a section is called standard if its restriction to the maximal compact subgroup $K^{\prime} \subset G_{\mathbb{A}}^{\prime}$ is independent of $s$ and factorizable if $\Phi(s)=\otimes_{p} \Phi_{p}(s)$ for the decomposition of the induced representation $I(s, \chi)=\otimes_{p}^{\prime} I_{p}\left(s, \chi_{p}\right)$. Here, for each prime $p, I_{p}\left(s, \chi_{p}\right)$ is the corresponding induced representation of $G_{p}^{\prime}$, the metaplectic extension of $\mathrm{SL}_{2}\left(\mathbb{Q}_{p}\right)$. The Eisenstein series associated to a standard section $\Phi(s) \in I(s, \chi)$ is given by

$$
E\left(g^{\prime}, s, \Phi\right)=\sum_{\gamma \in P_{\mathbb{Q}}^{\prime} \backslash G_{\mathbb{Q}}^{\prime}} \Phi\left(\gamma g^{\prime}, s\right) .
$$

This series is absolutely convergent for $\operatorname{Re}(s)>1$ and has a meromorphic continuation to the whole complex $s$-plane. Note that this series is normalized so that it has a functional equation

$$
E\left(g^{\prime},-s, M(s) \Phi\right)=E\left(g^{\prime}, s, \Phi\right),
$$

where $M(s): I(s, \chi) \rightarrow I\left(-s, \chi^{-1}\right)$ is the intertwining operator. It has a Fourier expansion

$$
E\left(g^{\prime}, s, \Phi\right)=\sum_{m \in \mathbb{Q}} E_{m}\left(g^{\prime}, s, \Phi\right),
$$

where, in the half-plane of absolute convergence,

$$
E_{m}\left(g^{\prime}, s, \Phi\right)=\int_{\mathbb{Q} \backslash \mathbb{A}} E\left(n(b) g^{\prime}, s, \Phi\right) \psi(-m b) d b,
$$

for $d b$ the self-dual measure on $\mathbb{A}$ with respect to $\psi$. When $m \neq 0$ and $\Phi(s)=\otimes_{p} \Phi_{p}(s)$ is factorizable, the $m$ th Fourier coefficient has a product expansion

$$
E_{m}\left(g^{\prime}, s, \Phi\right)=\prod_{p \leqslant \infty} W_{m, p}\left(g_{p}^{\prime}, s, \Phi_{p}\right)
$$

where

$$
W_{m, p}\left(g_{p}^{\prime}, s, \Phi_{p}\right)=\int_{\mathbb{Q}_{p}} \Phi_{p}\left(w n(b) g_{p}^{\prime}, s\right) \psi(-m b) d b
$$

is the local Whittaker function and

$$
w=\left(\begin{array}{cc}
0 & -1 \\
1 & 0
\end{array}\right) \in G_{\mathbb{Q}}^{\prime}
$$




\section{DeRivatives of Eisenstein SERIES AND FALtings Heights}

Here $d b$ is the self dual measure on $\mathbb{Q}_{p}$ for $\psi_{p}$. On the other hand, the constant term is

$$
E_{0}\left(g^{\prime}, s, \Phi\right)=\Phi\left(g^{\prime}, s\right)+\prod_{p \leqslant \infty} W_{0, p}\left(g_{p}^{\prime}, s, \Phi\right) .
$$

Recall that the poles of the Eisenstein series are precisely those of its constant term.

In this paper, we are only concerned with the case of a quadratic character $\chi$ given by $\chi(x)=$ $(x, \kappa)_{\mathbb{A}}$ for $\kappa \in \mathbb{Q}^{\times}$, where $(\cdot, \cdot)_{\mathbb{A}}$ denotes the global quadratic Hilbert symbol, so we omit $\chi$ from the notation and write $I(s)=\otimes_{p}^{\prime} I_{p}(s)$ for the induced representation, etc. We now begin to make specific choices of the local sections $\Phi_{p}(s)$.

As before, let $B$ be an indefinite quaternion algebra over $\mathbb{Q}$ with a fixed maximal order $O_{B}$. Once again, the case $B=M_{2}(\mathbb{Q})$ and $O_{B}=M_{2}(\mathbb{Z})$ will be allowed. Let

$$
V=\left\{x \in B \mid \operatorname{tr}^{o}(x)=0\right\}
$$

with quadratic form defined by $Q(x)=-x^{2}$ and let $L=O_{B} \cap V$. Note that the determinant of the quadratic space $(V, Q)$, i.e. $\operatorname{det}(S)$ where $S$ is the matrix for the quadratic form, is a square. Therefore, the discriminant $-\operatorname{det}(S)$ is -1 and the quadratic character $\chi_{V}$ associated with $V$ is given by $\chi_{V}(x)=(x,-1)_{\mathbb{A}}$. We therefore take $\chi=\chi_{V}$ and $\kappa=-1$ in this case.

The group $G_{\mathbb{A}}^{\prime}\left(\right.$ respectively $\left.G_{p}^{\prime}\right)$ acts on the Schwartz space $S\left(V(\mathbb{A})\right.$ ) (respectively $S\left(V_{p}\right)$ ) via the Weil representation $\omega$ (respectively $\omega_{p}$ ) determined by $\psi$ (respectively $\psi_{p}$ ).

For a finite prime $p$, let $\Phi_{p}(s) \in I_{p}(s)$ be the standard section extending $\lambda_{p}\left(\varphi_{p}\right)$, where

$$
\lambda_{p}: S\left(V_{p}\right) \rightarrow I_{p}\left(\frac{1}{2}\right), \quad \lambda_{p}\left(\varphi_{p}\right)\left(g^{\prime}\right)=\left(\omega\left(g^{\prime}\right) \varphi_{p}\right)(0)
$$

is the usual map and $\varphi_{p} \in S\left(V_{p}\right)$ is the characteristic function of $L_{p}=L \otimes_{\mathbb{Z}} \mathbb{Z}_{p}$.

Let $K_{\infty}^{\prime}$ be the inverse image in $G_{\mathbb{A}}^{\prime}$ of $\mathrm{SO}(2) \subset \mathrm{SL}_{2}(\mathbb{R})$. For $\ell \in \frac{1}{2} \mathbb{Z}$, there is a character $\nu_{\ell}$ of $K_{\infty}^{\prime}$ such that

$$
\nu_{\ell}\left(\left[k_{\theta}, 1\right]\right)^{2}=e^{2 i \theta \ell} .
$$

For $\ell \in \frac{3}{2}+2 \mathbb{Z}$, there is a unique standard section $\Phi_{\infty}^{\ell}(s) \in I_{\infty}(s)$ with

$$
\Phi_{\infty}^{\ell}(k, s)=\nu_{\ell}(k),
$$

for $k \in K_{\infty}^{\prime}$.

Let

$$
\Phi^{\ell, D(B)}(s)=\Phi_{\infty}^{\ell}(s) \otimes\left(\otimes_{p} \Phi_{p}(s)\right)
$$

be the associated global standard section. A little more generally, for a finite prime $p$, let $\Phi_{p}^{+}(s)$ be the standard section arising from the maximal order $M_{2}\left(\mathbb{Z}_{p}\right)$ in $M_{2}\left(\mathbb{Q}_{p}\right)$ and let $\Phi_{p}^{-}(s)$ be the standard section arising from the maximal order in the division quaternion algebra over $\mathbb{Q}_{p}$. Then, for any square free positive integer $D$, we have a global section

$$
\Phi^{\ell, D}(s)=\Phi_{\infty}^{\ell}(s) \otimes\left(\otimes_{p} \Phi_{p}^{\epsilon_{p}(D)}(s)\right),
$$

where $\epsilon_{p}(D)=(-1)^{\operatorname{ord}_{p}(D)}$.

Since, by strong approximation, $G_{\mathbb{A}}^{\prime}=G_{\mathbb{Q}}^{\prime} G_{\mathbb{R}}^{\prime} K^{0}$ for any open subgroup $K^{0}$ of $G_{\mathbb{A}_{f}}^{\prime}$, we lose no information by restricting automorphic forms to $G_{\mathbb{R}}^{\prime}$, the inverse image of $\mathrm{SL}_{2}(\mathbb{R})$ in $G_{\mathbb{A}}^{\prime}$. For $\tau=$ $u+i v \in \mathfrak{H}$, let

$$
g_{\tau}^{\prime}=\left[n(u) m\left(v^{\frac{1}{2}}\right), 1\right] \in G_{\infty}^{\prime} \subset G_{\mathbb{A}}^{\prime} .
$$

Then, if $\Phi(s)$ is a standard factorizable section with $\Phi_{\infty}(s)=\Phi_{\infty}^{\ell}(s)$, we set

$$
E(\tau, s, \Phi)=v^{-\ell / 2} E\left(g_{\tau}^{\prime}, s, \Phi\right),
$$




\section{S. S. Kudla, M. RAPOport And T. YAnG}

and, by (6.10), we have

$$
E_{m}(\tau, s, \Phi)=v^{-\ell / 2} W_{m, \infty}\left(g_{\tau}^{\prime}, s, \Phi_{\infty}^{\ell}\right) \cdot \prod_{p} W_{m, p}\left(s, \Phi_{p}\right)
$$

for $m \neq 0$, and

$$
E_{0}(\tau, s, \Phi)=v^{\frac{1}{2}(s+1-\ell)} \cdot \Phi_{f}(e)+v^{-\ell / 2} W_{0, \infty}\left(g_{\tau}^{\prime}, s, \Phi_{\infty}^{\ell}\right) \prod_{p} W_{0, p}\left(s, \Phi_{p}\right) .
$$

The main series of interest to us will be $E\left(\tau, s, \Phi^{\ell, D}\right)$, associated to the standard section $\Phi^{\ell, D}(s)$ of (6.18). This series has weight $\ell$, where $\ell=\frac{3}{2}, \frac{7}{2}, \frac{11}{2}, \ldots$ Note that the character $\chi$ is given by $\chi(x)=(x,-1)_{\mathbb{A}}$ in this case. A second family $E\left(\tau, s, \Phi^{\ell, D}\right)$, with $\ell=\frac{1}{2}, \frac{5}{2}, \frac{9}{2}, \ldots$ etc. is obtained by the same construction applied to the quadratic space $\left(V, Q_{-}\right)$, where $Q_{-}(x)=x^{2}$. In this case, $\kappa=1$ and $\chi$ is trivial. These cases will be discussed in more detail in [KY]. In the present paper, we will only be concerned with the case $\ell=\frac{3}{2}$, and so, from now on, we take $\kappa=-1$.

In the next section, we give a geometric interpretation of the first two terms of the Laurent expansion of the series $E\left(\tau, s, \Phi^{\frac{3}{2}, D(B)}\right)$ at the point $s=\frac{1}{2}$. For this it will be convenient to normalize the series as follows. For any square free positive integer $D$, let

$$
\mathbb{E}(\tau, s ; D):=\left(s+\frac{1}{2}\right) c(D) \Lambda_{D}(2 s+1) E\left(\tau, s, \Phi^{\frac{3}{2}, D}\right),
$$

where

$$
\Lambda_{D}(2 s+1)=\left(\frac{D}{\pi}\right)^{s+\frac{1}{2}} \Gamma\left(s+\frac{1}{2}\right) \zeta(2 s+1) \cdot \prod_{p \mid D}\left(1-p^{-2 s-1}\right)
$$

and

$$
c(D)=-(-1)^{\operatorname{ord}(D)} \frac{1}{2 \pi} D \prod_{p \mid D}(p+1)^{-1},
$$

where $\operatorname{ord}(D)=\sum_{p} \operatorname{ord}_{p}(D)$. Note that at the point $s=\frac{1}{2}$, of interest to us, the normalizing factor has the value

$$
c(D) \Lambda_{D}(2)=-(-1)^{\operatorname{ord}(D)} \frac{1}{12} \prod_{p \mid D}(p-1) .
$$

Then, in the case $D=D(B)$, and recalling (2.7),

$$
c(D) \Lambda_{D}(2)=-\operatorname{vol}(\mathcal{M}(\mathbb{C})) .
$$

This expression explains the choice of $c(D)$. In addition, the normalized Eisenstein series satisfies the simple functional equation (cf. $\S 15$ ),

$$
\mathbb{E}(\tau, s ; D)=\mathbb{E}(\tau,-s ; D) .
$$

Finally, we restrict to the case $D=D(B)>1$ and introduce the modified Eisenstein series

$$
\mathcal{E}(\tau, s ; D(B)):=\mathbb{E}(\tau, s ; D(B))+\sum_{p \mid D} c_{p}(s) \mathbb{E}(\tau, s ; D(B) / p),
$$

where $c_{p}(s)$ is any rational function of $p^{-s}$ satisfying

$$
c_{p}\left(\frac{1}{2}\right)=0 \quad \text { and } \quad c_{p}^{\prime}\left(\frac{1}{2}\right)=-\frac{p-1}{p+1} \log (p) .
$$

To retain the functional equation (6.28), one should also require that $c_{p}(s)=c_{p}(-s)$, although we will not use this. The motivation for the definition of $\mathcal{E}(\tau, s, D(B))$ comes from geometric considerations which will emerge below. Note that

$$
\mathcal{E}\left(\tau, \frac{1}{2} ; D(B)\right)=\mathbb{E}\left(\tau, \frac{1}{2} ; D(B)\right),
$$




\section{DeRivatives of Eisenstein SERIES AND FALtings Heights}

and

$$
\mathcal{E}^{\prime}\left(\tau, \frac{1}{2} ; D(B)\right)=\mathbb{E}^{\prime}\left(\tau, \frac{1}{2} ; D(B)\right)+\sum_{p \mid D} c_{p}^{\prime}\left(\frac{1}{2}\right) \cdot \mathbb{E}\left(\tau, \frac{1}{2} ; D(B) / p\right)
$$

\section{The main identities}

In this section, we state our main results on the generating functions

$$
\phi_{\operatorname{deg}}(\tau)=-\operatorname{vol}(\mathcal{M}(\mathbb{C}))+\sum_{m>0} \operatorname{deg}\left(\mathcal{Z}(m)_{\mathbb{C}}\right) q^{m}
$$

and

$$
\phi_{\text {height }}(\tau)=\sum_{m}\langle\hat{\mathcal{Z}}(m, v), \hat{\omega}\rangle q^{m}
$$

introduced in $\S 5$.

The following result is actually well known (cf. for example, [Fun02]). We state it here to bring out the analogy with Theorem 7.2.

Proposition 7.1. For any indefinite quaternion division algebra $B$ over $\mathbb{Q}$ with associated moduli stack $\mathcal{M}$, as in $\S \S 1-5$ above, the generating function for the degrees of the special cycles coincides with the value at $s=\frac{1}{2}$ of the Eisenstein series $\mathcal{E}(\tau, s ; D(B))$ of weight $\frac{3}{2}$ :

$$
\phi_{\operatorname{deg}}(\tau)=\mathcal{E}\left(\tau, \frac{1}{2} ; D(B)\right) .
$$

TheOREM 7.2. Under the same assumptions, the generating series for heights of the special cycles coincides with the derivative at $s=\frac{1}{2}$ of the Eisenstein series $\mathcal{E}(\tau, s ; D(B))$ of weight $\frac{3}{2}$ :

$$
\phi_{\text {height }}(\tau)=\mathcal{E}^{\prime}\left(\tau, \frac{1}{2} ; D(B)\right) \text {. }
$$

These identities are to be understood as follows. We write the Fourier expansion of the modified Eisenstein series as

$$
\mathcal{E}(\tau, s ; D(B))=\sum_{m} A_{m}(s, v) q^{m}
$$

so that the Fourier expansions of the value and derivative at $s=\frac{1}{2}$ are

$$
\mathcal{E}\left(\tau, \frac{1}{2} ; D(B)\right)=\sum_{m} A_{m}\left(\frac{1}{2}, v\right) q^{m}
$$

and

$$
\mathcal{E}^{\prime}\left(\tau, \frac{1}{2} ; D(B)\right)=\sum_{m} A_{m}^{\prime}\left(\frac{1}{2}, v\right) q^{m} .
$$

Proposition 7.1 then says that

$$
A_{m}\left(\frac{1}{2}, v\right)= \begin{cases}\operatorname{deg}\left(\mathcal{Z}(m)_{\mathbb{C}}\right) & \text { if } m>0 \\ -\operatorname{vol}(\mathcal{M}(\mathbb{C})) & \text { if } m=0 \\ 0 & \text { if } m<0\end{cases}
$$

Analogously, Theorem 7.2 says that

$$
A_{m}^{\prime}\left(\frac{1}{2}, v\right)=\langle\hat{\mathcal{Z}}(m, v), \hat{\omega}\rangle
$$

As already explained in the introduction, Theorem 7.2 is proved by an explicit computation of both sides of (7.7). For the left-hand side, this will be done in the next section. The right-hand side will be computed in $\S \S 9-12$. 


\section{S. S. Kudla, M. Rapoport And T. YAng}

\section{Fourier expansions and derivatives}

In this section, we describe the first two terms of the Laurent expansion of the Eisenstein series $\mathcal{E}(\tau, s ; D(B))$ at the point $s=\frac{1}{2}$. By (6.21) and (6.22), the essential point is to describe the behaviour of the local Whittaker functions $W_{m, p}\left(s, \Phi_{p}^{D}\right)$ and

$$
W_{m, \infty}\left(\tau, s, \Phi_{\infty}^{\frac{3}{2}}\right):=v^{-\frac{3}{4}} W_{m, \infty}\left(g_{\tau}^{\prime}, s, \Phi_{\infty}^{\frac{3}{2}}\right) .
$$

The calculations of this section will be elementary manipulations based on results about these Whittaker functions quoted from Part IV below.

In what follows, for a non-zero integer $m$, we write

$$
4 m=n^{2} d,
$$

where $-d$ is a fundamental discriminant, i.e. discriminant of the field $k=k_{d}=\mathbb{Q}(\sqrt{-m})$. Note that if $4 m=-n^{2}$, then $k=\mathbb{Q} \oplus \mathbb{Q}$. Let $\chi_{d}$ be the corresponding Dirichlet character, so that

$$
\chi_{d}(p)=\left\{\begin{aligned}
1 & \text { if } p \text { is split in } k_{d}, \\
-1 & \text { if } p \text { is inert in } k_{d}, \\
0 & \text { if } p \text { is ramified in } k_{d} .
\end{aligned}\right.
$$

For a given $m$ and for a square free positive integer $D$, define a modification of the standard Dirichlet $L$-series $L\left(s, \chi_{d}\right)$ by

$$
L\left(s, \chi_{m} ; D\right):=L\left(s, \chi_{d}\right) \prod_{p \mid n D} b_{p}(n, s ; D),
$$

where $b_{p}(n, s ; D)$ is defined as follows. Set

$$
k=k_{p}(n)=\operatorname{ord}_{p}(n)
$$

and $X=p^{-s}$. Then for $p \nmid D$

$$
b_{p}(n, s ; D)=\frac{1-\chi_{d}(p) X+\chi_{d}(p) p^{k} X^{(1+2 k)}-\left(p X^{2}\right)^{k+1}}{1-p X^{2}},
$$

and, for $p \mid D$,

$$
b_{p}(n, s ; D)=\frac{(1-\chi X)\left(1-p^{2} X^{2}\right)-\chi p^{k+1} X^{2 k+1}+p^{k+2} X^{2 k+2}+\chi p^{k+1} X^{2 k+3}-p^{2 k+2} X^{2 k+4}}{1-p X^{2}} .
$$

Here, for a moment, we write $\chi$ for $\chi_{d}(p)$. Depending on whether or not $p \mid d$ we can rewrite (8.7) as

$$
b_{p}(n, s ; D)=\frac{1-p^{2} X^{2}+p^{k+2} X^{2 k+2}\left(1-X^{2}\right)}{1-p X^{2}}, \quad \text { if } p \mid d \text { and } p \mid D,
$$

and

$$
b_{p}(n, s ; D)=\frac{(1-\chi X)\left(1-p^{2} X^{2}\right)-\chi p^{k+1} X^{2 k+1}(1-\chi p X)\left(1-X^{2}\right)}{1-p X^{2}}, \quad \text { if } p \nmid d \text { and } p \mid D .
$$

In all cases the local factor $b_{p}(n, s ; D)$ is, in fact, a polynomial in $X=p^{-s}$ and is, hence, entire in $s$. It satisfies the functional equation

$$
|n D|_{p}^{-s} b_{p}(n, s ; D)=|n D|_{p}^{s-1} b_{p}(n, 1-s ; D)
$$

One of the main results of $\S 13$ is the following. 


\section{DeRivatives of Eisenstein SERIES AND FALtings Heights}

Proposition 8.1. Fix a prime $p$.

i) If $m \neq 0$, then

$$
W_{m, p}\left(s+\frac{1}{2}, \Phi_{p}^{D}\right)=L_{p}\left(s+1, \chi_{d}\right) b_{p}(n, s+1 ; D) \cdot \begin{cases}C_{p}^{+} \cdot \zeta_{p}(2 s+2)^{-1} & \text { if } p \nmid D, \\ C_{p}^{-} & \text {if } p \mid D,\end{cases}
$$

where the constants $C_{p}^{ \pm}$are given by

$$
C_{p}^{+}= \begin{cases}1 & \text { if } p \neq 2 \\ \frac{1}{\sqrt{2}} \zeta_{8}^{-1} & \text { if } p=2\end{cases}
$$

and $C_{p}^{-}=-p^{-1} C_{p}^{+}$. Here $\zeta_{8}=e\left(\frac{1}{8}\right)$.

ii) If $m=0$, then

$$
W_{0, p}\left(s+\frac{1}{2}, \Phi_{p}^{D}\right)=\zeta_{p}(2 s) \cdot \begin{cases}C_{p}^{+} \cdot \zeta_{p}(2 s+1)^{-1} & \text { if } p \nmid D \\ C_{p}^{-} \cdot \zeta_{p}(2 s-1)^{-1} & \text { if } p \mid D .\end{cases}
$$

From (6.21), (6.22) and these formulas, we obtain a nice description of the Fourier expansion of $\mathbb{E}(\tau, s ; D)$.

Corollary 8.2. Let $C_{f}(D)=\prod_{p} C_{p}^{\epsilon_{p}(D)}$.

i) For $m \neq 0$,

$$
E_{m}(\tau, s ; D)=C_{f}(D) \cdot W_{m, \infty}\left(\tau, s, \Phi_{\infty}^{\frac{3}{2}}\right) \cdot \frac{L\left(s+\frac{1}{2}, \chi_{d}\right)}{\zeta_{D}(2 s+1)} \cdot(n D)^{-2 s} \prod_{p} b_{p}\left(n, \frac{1}{2}-s ; D\right),
$$

and

$$
\begin{aligned}
\mathbb{E}_{m}(\tau, s ; D)= & c(D) C_{f}(D)\left(\frac{D}{\pi}\right)^{s+\frac{1}{2}} \Gamma\left(s+\frac{3}{2}\right) \cdot W_{m, \infty}\left(\tau, s, \Phi_{\infty}^{\frac{3}{2}}\right) \\
& \times L\left(s+\frac{1}{2}, \chi_{d}\right) \cdot(n D)^{-2 s} \prod_{p} b_{p}\left(n, \frac{1}{2}-s ; D\right) .
\end{aligned}
$$

ii) For $m=0$,

$$
E_{0}(\tau, s ; D)=v^{\frac{1}{2}\left(s-\frac{1}{2}\right)}+W_{0, \infty}\left(\tau, s, \Phi_{\infty}^{\frac{3}{2}}\right) C_{f}(D) \cdot \frac{\zeta(2 s)}{\zeta_{D}(2 s+1)} \cdot \prod_{p \mid D} \frac{1}{\zeta_{p}(2 s-1)},
$$

and

$$
\begin{aligned}
\mathbb{E}_{0}(\tau, s ; D)= & v^{\frac{1}{2}\left(s-\frac{1}{2}\right)}\left(s+\frac{1}{2}\right) c(D) \Lambda_{D}(2 s+1) \\
& +W_{0, \infty}\left(\tau, s, \Phi_{\infty}^{\frac{3}{2}}\right) c(D) C_{f}(D)\left(\frac{D}{\pi}\right)^{s+\frac{1}{2}} \Gamma\left(s+\frac{3}{2}\right) \cdot \zeta(2 s) \cdot \prod_{p \mid D} \frac{1}{\zeta_{p}(2 s-1)} .
\end{aligned}
$$

Here

$$
c(D) C_{f}(D)=-\frac{1}{\sqrt{2}} \zeta_{8}^{-1} \frac{1}{2 \pi} \prod_{p \mid D}(p+1)^{-1} .
$$

Using Corollary 8.2, we now compute the value of $\mathbb{E}(\tau, s ; D)$ at $s=\frac{1}{2}$. We start with the constant term.

The following result is a special case of part iii of Proposition 14.1 below. 


\section{S. S. Kudla, M. RAPOPORT And T. YANG}

LEMMA 8.3.

$$
W_{0, \infty}\left(\tau, s, \Phi_{\infty}^{\frac{3}{2}}\right)=2 \pi(-i)^{\frac{3}{2}} v^{-\frac{1}{2}\left(s+\frac{1}{2}\right)} 2^{-s} \frac{\Gamma(s)}{\Gamma(\alpha) \Gamma(\beta)},
$$

for $\alpha=\frac{1}{2}\left(s+\frac{5}{2}\right)$ and $\beta=\frac{1}{2}\left(s-\frac{1}{2}\right)$. Here $(-i)^{\frac{3}{2}}=e\left(-\frac{3}{8}\right)$.

Since the zero of $\Gamma(\beta)^{-1}$ at $s=\frac{1}{2}$ cancels the pole of $\zeta(2 s)$ there, the second term in $\mathbb{E}_{0}(\tau, s ; D)$ has a zero of order equal to the number of primes dividing $D$ and we obtain the following.

Corollary 8.4. For $D>1$, the constant term at $s=\frac{1}{2}$ is

$$
\mathbb{E}_{0}\left(\tau, \frac{1}{2} ; D\right)=c(D) \Lambda_{D}(2)=-(-1)^{\operatorname{ord}(D)} \frac{1}{12} \prod_{p \mid D}(p-1)=\zeta_{D}(-1) .
$$

Next we consider the coefficients of $\mathbb{E}\left(\tau, \frac{1}{2} ; D\right)$ for $m \neq 0$.

If $p \nmid D$, then by (8.6),

$$
b_{p}(n, 0 ; D)=\frac{1-\chi_{d}(p)+\chi_{d}(p) p^{k}-p^{k+1}}{1-p} .
$$

Note that, when $\chi_{d}(p)=1$, this simplifies to $p^{k}=|n|_{p}^{-1}$.

If $p \mid D$, then by (8.7)-(8.9),

$$
b_{p}(n, 0 ; D)=\left(1-\chi_{d}(p)\right)(1+p) .
$$

Note that this quantity is actually independent of $n$ and that $b_{p}(n, 0 ; D)=0$ if and only if $p \mid D$ and $\chi_{d}(p)=1$.

The proof of the following identity is a simple combinatorial exercise, which we omit.

LEMMA 8.5.

i) For $p \nmid D$ and $k=\operatorname{ord}_{p}(n)$,

$$
b_{p}(n, 0 ; D)=\sum_{c \mid p^{k}} c \prod_{\ell \mid c}\left(1-\chi_{d}(\ell) \ell^{-1}\right),
$$

where $\ell$ runs over the prime factors of $c$ and the product is given the value 1 when $c=1$.

ii)

$$
\prod_{p \nmid D} b_{p}(n, 0 ; D)=\sum_{\substack{c \mid n \\(c, D)=1}} c \prod_{\ell \mid c}\left(1-\chi_{d}(\ell) \ell^{-1}\right) .
$$

Here, again, $\ell$ runs over the prime factors of $c$ and the product is given the value 1 when $c=1$.

On the other hand, the following fact is a special case of part iv of Proposition 14.1 below. LEMMA 8.6.

$$
W_{m, \infty}\left(\tau, \frac{1}{2}, \Phi_{\infty}^{\frac{3}{2}}\right)= \begin{cases}2 C_{\infty} \cdot m^{\frac{1}{2}} q^{m} & \text { if } m>0 \\ 0 & \text { otherwise }\end{cases}
$$

where $C_{\infty}=(-2 i)^{\frac{3}{2}} \pi$.

Combining these facts, we obtain the following results.

For $m<0$, the vanishing of the archimedean factor yields:

$$
\mathbb{E}_{m}\left(\tau, \frac{1}{2} ; D\right)=0, \quad \text { when } \chi_{d} \neq 1 \text {, or } D>1 \text {. }
$$




\section{DeRivatives of Eisenstein SERIES AND FALtings Heights}

For $m>0$, part i of Corollary 8.2 gives

$$
\begin{aligned}
\mathbb{E}_{m}\left(\tau, \frac{1}{2} ; D\right)= & c(D) C_{f}(D) C_{\infty} \cdot \frac{D}{\pi} \cdot 2 m^{\frac{1}{2}} q^{m} \cdot L\left(1, \chi_{d}\right)(n D)^{-1} \prod_{p} b_{p}(n, 0 ; D) \\
= & c(D) C_{f}(D) C_{\infty} \cdot q^{m} \cdot 2 \frac{h(d)}{w(d)} \cdot\left(\sum_{\substack{c \mid n \\
(c, D)=1}} c \prod_{\ell \mid c}\left(1-\chi_{d}(\ell) \ell^{-1}\right)\right) \\
& \times\left(\prod_{p \mid D}\left(1-\chi_{d}(p)\right)(1+p)\right) \\
= & q^{m} \cdot 2 \frac{h(d)}{w(d)} \cdot\left(\sum_{\substack{c \mid n \\
(c, D)=1}} c \prod_{\ell \mid c}\left(1-\chi_{d}(\ell) \ell^{-1}\right)\right) \cdot\left(\prod_{p \mid D}\left(1-\chi_{d}(p)\right)\right) .
\end{aligned}
$$

Here, $w(d)=\left|O_{k}^{\times}\right|$is the number of roots of unity in the maximal order $O_{k}$ of $k_{d}, h(d)$ is the class number, and

$$
c(D) C_{f}(D) C_{\infty}=\prod_{p \mid D}(p+1)^{-1} .
$$

For $m>0$, let

$$
H_{0}(m ; D)=\frac{h(d)}{w(d)} \cdot \sum_{\substack{c \mid n \\(c, D)=1}} c \prod_{\ell \mid c}\left(1-\chi_{d}(\ell) \ell^{-1}\right),
$$

where, as before, in the product $\ell$ runs over the prime factors of $c$, the product is taken to be 1 when $c=1$, and

$$
\delta(d ; D)=\prod_{p \mid D}\left(1-\chi_{d}(p)\right) .
$$

Thus, we obtain the Fourier expansion of $\mathcal{E}\left(\tau, \frac{1}{2} ; D\right)$.

Proposition 8.7. For $D>1$,

$$
\mathcal{E}\left(\tau, \frac{1}{2} ; D\right)=c(D) \Lambda_{D}(2)+\sum_{m>0} 2 \delta(d ; D) H_{0}(m ; D) q^{m}
$$

Here

$$
c(D) \Lambda_{D}(2)=-(-1)^{\operatorname{ord}(D)} \frac{1}{12} \prod_{p \mid D}(p-1) .
$$

This Eisenstein series of weight $\frac{3}{2}$ is a familiar object. Recall that, if $O_{c^{2} d}$ is the order in $O_{k}$ of conductor $c$, with class number $h\left(c^{2} d\right)$ and with $w\left(c^{2} d\right)=\left|O_{c^{2} d}^{\times}\right|$, then [BS66, p. 250] $]^{2}$

$$
\frac{h\left(c^{2} d\right)}{w\left(c^{2} d\right)}=\frac{h(d)}{w(d)} \cdot c \prod_{\ell \mid c}\left(1-\chi_{d}(\ell) \ell^{-1}\right) .
$$

Thus,

$$
H_{0}(m ; D)=\sum_{\substack{c \mid n \\(c, D)=1}} \frac{h\left(c^{2} d\right)}{w\left(c^{2} d\right)}
$$

\footnotetext{
${ }^{2}$ The quantity $e_{c}$ there is $\left|\mathcal{O}_{d}^{\times}: \mathcal{O}_{c^{2} d}^{\times}\right|=w(d) / w\left(c^{2} d\right)$.
} 


\section{S. S. Kudla, M. Rapoport And T. YAng}

For example, if $D=1$, i.e. in the case of $B=M_{2}(\mathbb{Q})$,

$$
H_{0}(m ; 1)=\sum_{c \mid n} \frac{h\left(c^{2} d\right)}{w\left(c^{2} d\right)}
$$

is quite close to ${ }^{3}$ the 'class number' $H(m)$ which appears in the Fourier expansion

$$
\mathcal{F}(\tau)=-\frac{1}{12}+\sum_{m>0} H(m) q^{m}+\sum_{n \in \mathbb{Z}} \frac{1}{16 \pi} v^{-\frac{1}{2}} \int_{1}^{\infty} e^{-4 \pi n^{2} v r} r^{-\frac{3}{2}} d r q^{-n^{2}}
$$

of Zagier's non-holomorphic Eisenstein series of weight $\frac{3}{2}$ [Coh75, Zag75]. In fact, when $D=1$, we have

$$
\mathcal{E}\left(\tau, \frac{1}{2} ; 1\right)=-\frac{1}{12}+\sum_{m>0} 2 H_{0}(m ; 1) q^{m}+\sum_{n \in \mathbb{Z}} \frac{1}{8 \pi} v^{-\frac{1}{2}} \int_{1}^{\infty} e^{-4 \pi n^{2} v r} r^{-\frac{3}{2}} d r \cdot q^{-n^{2}}
$$

This case will be discussed in detail in the sequel to this paper [KRY].

Next we consider the derivative $\mathcal{E}^{\prime}\left(\tau, \frac{1}{2} ; D\right)$ in the case $D=D(B)>1$. In this case, the only terms which contribute are the following:

i) $m>0$ and $\delta(d ; D) \neq 0$;

ii) $m>0$ and there is a unique $p \mid D(B)$ such that $\chi_{d}(p)=1$;

iii) $m<0$ and $\delta(d ; D) \neq 0$; and

iv) $m=0$.

In cases i and iv, $\mathcal{E}_{m}\left(\tau, \frac{1}{2} ; D\right) \neq 0$. In cases ii and iii, $\mathcal{E}_{m}(\tau, s ; D)$ has a simple zero at $s=\frac{1}{2}$ due to the vanishing of the local factor $b_{p}(n, 0 ; D)$ in case ii and the archimedean factor $W_{m, \infty}\left(\tau, \frac{1}{2} ; \Phi_{\infty}^{\frac{3}{2}}\right)$ in case iii. In all other cases, $\mathcal{E}_{m}\left(\tau, \frac{1}{2} ; D\right)$ has a zero of order at least two at $s=\frac{1}{2}$.

Theorem 8.8. Assume that $D=D(B)>1$.

i) If $m>0$ and there is no prime $p \mid D$ for which $\chi_{d}(p)=1$, then

$$
\begin{aligned}
\mathcal{E}_{m}^{\prime}\left(\tau, \frac{1}{2} ; D\right)= & 2 \delta(d ; D) H_{0}(m ; D) \cdot q^{m} \cdot\left[\frac{1}{2} \log (d)+\frac{L^{\prime}\left(1, \chi_{d}\right)}{L\left(1, \chi_{d}\right)}-\frac{1}{2} \log (\pi)-\frac{1}{2} \gamma\right. \\
& \left.+\frac{1}{2} J(4 \pi m v)+\sum_{\substack{p \\
p \nmid D}}\left(\log |n|_{p}-\frac{b_{p}^{\prime}(n, 0 ; D)}{b_{p}(n, 0 ; D)}\right)+\sum_{\substack{p \\
p \mid D}} K_{p} \log (p)\right] .
\end{aligned}
$$

Here

$$
K_{p}= \begin{cases}-k+\frac{(p+1)\left(p^{k}-1\right)}{2(p-1)} & \text { if } \chi_{d}(p)=-1, \\ -1-k+\frac{p^{k+1}-1}{p-1} & \text { if } \chi_{d}(p)=0,\end{cases}
$$

with $k=\operatorname{ord}_{p}(n)$, and

$$
J(t)=\int_{0}^{\infty} e^{-t r}\left[(1+r)^{\frac{1}{2}}-1\right] r^{-1} d r .
$$

An explicit expression for the logarithmic derivative of $b_{p}(n, s ; D)$ is given by part $i$ of Lemma 8.10, and $H_{0}(m ; D)$ and $\delta(d ; D)$ are given by (8.17) and (8.18), respectively.

\footnotetext{
${ }^{3}$ Precisely, $2 H_{0}(m ; 1)=H(4 m)$.
} 


\section{DeRivatives of Eisenstein SERIES AND FALtings Heights}

ii) If there is a unique prime $p \mid D$ such that $\chi_{d}(p)=1$, then for $k=\operatorname{ord}_{p}(n)$,

$$
\mathcal{E}_{m}^{\prime}\left(\tau, \frac{1}{2} ; D\right)=2 \delta(d ; D / p) H_{0}(m ; D) \cdot\left(p^{k}-1\right) \log (p) \cdot q^{m} .
$$

iii) If $m<0$, then

$$
\mathcal{E}_{m}^{\prime}\left(\tau, \frac{1}{2} ; D\right)=2 \delta(d ; D) H_{0}(m ; D) \cdot q^{m} \cdot \frac{1}{4 \pi}|m|^{-\frac{1}{2}} v^{-\frac{1}{2}} \int_{1}^{\infty} e^{-4 \pi|m| v r} r^{-\frac{3}{2}} d r,
$$

where, for $m<0, H_{0}(m ; D)$ is defined by (8.29) below.

iv)

$$
\mathcal{E}_{0}^{\prime}\left(\tau, \frac{1}{2} ; D\right)=c(D) \Lambda_{D}(2)\left[\frac{1}{2} \log (v)-2 \frac{\zeta^{\prime}(-1)}{\zeta(-1)}-1+2 C+\sum_{p \mid D} \frac{p \log (p)}{p-1}\right] .
$$

v) All other Fourier coefficients of $\mathcal{E}^{\prime}\left(\tau, \frac{1}{2} ; D\right)$ vanish.

Proof. We begin with the Eisenstein series $\mathbb{E}(\tau, s ; D)$ for any $D>1$.

First consider case i, so that $m>0$ and that there are no primes $p \mid D$ with $\chi_{d}(p)=1$. Then, using part i of Corollary 8.2, we have

$$
\begin{aligned}
\mathbb{E}_{m}^{\prime}\left(\tau, \frac{1}{2} ; D\right)=\mathbb{E}_{m}\left(\tau, \frac{1}{2} ; D\right)[\log (D)-\log (\pi)+1-\gamma & +\frac{W_{m, \infty}^{\prime}\left(\tau, \frac{1}{2}, \Phi^{\frac{3}{2}}\right)}{W_{m, \infty}\left(\tau, \frac{1}{2}, \Phi^{\frac{3}{2}}\right)} \\
& \left.+\frac{L^{\prime}\left(1, \chi_{d}\right)}{L\left(1, \chi_{d}\right)}-2 \log (n D)-\sum_{p} \frac{b_{p}^{\prime}(n, 0 ; D)}{b_{p}(n, 0 ; D)}\right] .
\end{aligned}
$$

The following fact is proved in $\S 14$.

LEMMA 8.9. For $m>0$,

$$
\frac{W_{m, \infty}^{\prime}\left(\tau, \frac{1}{2}, \Phi^{\frac{3}{2}}\right)}{W_{m, \infty}\left(\tau, \frac{1}{2}, \Phi^{\frac{3}{2}}\right)}=\frac{1}{2}\left[\log (\pi m)-\frac{\Gamma^{\prime}\left(\frac{3}{2}\right)}{\Gamma\left(\frac{3}{2}\right)}+J(4 \pi m v)\right] .
$$

Using this result and the fact that

$$
\frac{\Gamma^{\prime}\left(\frac{3}{2}\right)}{\Gamma\left(\frac{3}{2}\right)}=2-\gamma-2 \log (2)
$$

and recalling that $4 m=n^{2} d$, we obtain

$$
\begin{aligned}
\mathbb{E}_{m}^{\prime}\left(\tau, \frac{1}{2} ; D\right)= & \mathbb{E}_{m}\left(\tau, \frac{1}{2} ; D\right)\left[\frac{1}{2} \log (d)+\frac{L^{\prime}\left(1, \chi_{d}\right)}{L\left(1, \chi_{d}\right)}-\frac{1}{2} \log (\pi)-\frac{1}{2} \gamma+\frac{1}{2} J(4 \pi m v)\right. \\
& \left.+\sum_{\substack{p \\
p \nmid D}}\left(\log |n|_{p}-\frac{b_{p}^{\prime}(n, 0 ; D)}{b_{p}(n, 0 ; D)}\right)-\log (D)+\sum_{\substack{p \\
p \mid D}}\left(\log |n|_{p}-\frac{b_{p}^{\prime}(n, 0 ; D)}{b_{p}(n, 0 ; D)}\right)\right] .
\end{aligned}
$$

Next we note the explicit expressions for the logarithmic derivatives of the $b_{p}(n, s ; D)$ 's which will be useful later. 


\section{S. S. Kudla, M. Rapoport And T. YAng}

Lemma 8.10.

i) For a prime $p \nmid D$,

$$
\begin{aligned}
\frac{1}{\log p} \cdot \frac{b_{p}^{\prime}(n, 0 ; D)}{b_{p}(n, 0 ; D)}= & \frac{\chi_{d}(p)-\chi_{d}(p)(2 k+1) p^{k}+(2 k+2) p^{k+1}}{1-\chi_{d}(p)+\chi_{d}(p) p^{k}-p^{k+1}}-\frac{2 p}{1-p} \\
& = \begin{cases}\frac{p^{k}-1}{p^{k}(p-1)}-2 k & \text { if } \chi_{d}(p)=1, \\
-\frac{2 p\left(1-(k+1) p^{k}+k p^{k+1}\right)}{(p-1)\left(p^{k+1}-1\right)} & \text { if } \chi_{d}(p)=0, \\
-\frac{1+3 p-(2 k+1) p^{k}-3 p^{k+1}+2 k p^{k+2}}{(p-1)\left(p^{k+1}+p^{k}-2\right)} & \text { if } \chi_{d}(p)=-1 .\end{cases}
\end{aligned}
$$

ii) For a prime $p \mid D$ with $\chi_{d}(p) \neq 1$,

$$
\frac{1}{\log (p)} \cdot \frac{b_{p}^{\prime}(n, 0 ; D)}{b_{p}(n, 0 ; D)}= \begin{cases}-\frac{2 p\left(p^{k+1}-1\right)}{p^{2}-1} & \text { if } \chi_{d}(p)=0 \\ -\frac{2(1+p) p^{k+1}+p^{2}-4 p-1}{2\left(p^{2}-1\right)} & \text { if } \chi_{d}(p)=-1\end{cases}
$$

Here $k=\operatorname{ord}_{p}(n)$.

In case ii, $m>0$ and there is a unique prime $p \mid D$ such that $\chi_{d}(p)=1$. In this case, it is easy to verify

$$
b_{p}^{\prime}(n, 0 ; D)=\left(1+p-2 p^{k+1}\right) \log (p) .
$$

Then, with the notation introduced above and using (8.13), we have

$$
\mathbb{E}_{m}^{\prime}\left(\tau, \frac{1}{2} ; D\right)=-2 \delta(d ; D / p) H_{0}(m ; D) \cdot q^{m} \cdot(p+1)^{-1} \cdot\left(1+p-2 p^{k+1}\right) \log (p) .
$$

Recall that $k=\operatorname{ord}_{p}(n)$.

Finally, in case iii, we need another result which is proved in $\S 14$.

Lemma 8.11. For $m<0$ :

$$
\begin{aligned}
W_{m, \infty}^{\prime}\left(\tau, \frac{1}{2}, \Phi_{\infty}^{\frac{3}{2}}\right) & =C_{\infty}|m|^{\frac{1}{2}} q^{m} e^{-4 \pi|m| v} \int_{0}^{\infty} e^{-4 \pi|m| v r}(r+1)^{-1} r^{\frac{1}{2}} d r \\
& =C_{\infty} \cdot \frac{1}{4} q^{m} v^{-\frac{1}{2}} \int_{1}^{\infty} e^{-4 \pi|m| v r} r^{-\frac{3}{2}} d r
\end{aligned}
$$

Using the second expression of this lemma and of Corollary 8.2, part i, we have, for $m<0$,

$$
\begin{aligned}
\mathbb{E}_{m}^{\prime}\left(\tau, \frac{1}{2} ; D\right)= & c(D) C_{f}(D) \cdot \frac{D}{\pi} \cdot W_{m, \infty}^{\prime}\left(\tau, \frac{1}{2}, \Phi^{\frac{3}{2}}\right) \cdot L\left(1, \chi_{d}\right) \cdot(n D)^{-1} \prod_{p} b_{p}(n, 0 ; D) \\
= & c(D) C_{f}(D) C_{\infty} \cdot \frac{4 h(d) \log |\epsilon(d)|}{w(d)|d|^{\frac{1}{2}}} \cdot n^{-1}\left(\sum_{\substack{c \mid n \\
(c, D)=1}} c \prod_{\ell \mid c}\left(1-\chi_{d}(\ell) \ell^{-1}\right)\right) \\
& \times\left(\prod_{p \mid D}\left(1-\chi_{d}(p)\right)(1+p)\right) q^{m} \frac{1}{4 \pi} v^{-\frac{1}{2}} \int_{1}^{\infty} e^{-4 \pi|m| v r} r^{-\frac{3}{2}} d r
\end{aligned}
$$

where $\epsilon(d)$ is the fundamental unit of the real quadratic field $k_{d}=\mathbb{Q}(\sqrt{|d|})$. Using the value (8.16), 


\section{DeRIVATIVES OF EISENSTEIN SERIES AND FAltings Heights}

this can rewritten as

$$
\mathbb{E}_{m}^{\prime}\left(\tau, \frac{1}{2} ; D\right)=2 H_{0}(m ; D) \delta(d ; D) \cdot q^{m} \cdot \frac{1}{4 \pi}|m|^{-\frac{1}{2}} v^{-\frac{1}{2}} \int_{1}^{\infty} e^{-4 \pi|m| v r} r^{-\frac{3}{2}} d r,
$$

where

$$
\begin{aligned}
H_{0}(m ; D) & =\frac{h(d) \log |\epsilon(d)|}{w(d)} \cdot\left(\sum_{\substack{c \mid n \\
(c, D)=1}} c \prod_{\ell \mid c}\left(1-\chi_{d}(\ell) \ell^{-1}\right)\right) \\
& =\sum_{\substack{c \mid n \\
(c, D)=1}} h\left(c^{2} d\right) \cdot \frac{\log \left|\epsilon\left(c^{2} d\right)\right|}{w\left(c^{2} d\right)}
\end{aligned}
$$

is the analogue of (8.17) and (8.20) in the case of a real quadratic field, i.e. for $m<0$.

Finally, we consider the constant term using Corollary 8.2, part ii and noting that, for $D=D(B)$, the second term there has a zero of order at least two. This gives

$$
\begin{aligned}
\mathbb{E}_{0}^{\prime}\left(\tau, \frac{1}{2} ; D\right) & =c(D) \Lambda_{D}(2)\left[\frac{1}{2} \log (v)+1+2 \frac{\Lambda_{D}^{\prime}(2)}{\Lambda_{D}(2)}\right] \\
& =c(D) \Lambda_{D}(2)\left[\frac{1}{2} \log (v)+1+\log (D)-\log (\pi)-\gamma+2 \frac{\zeta^{\prime}(2)}{\zeta(2)}+2 \sum_{p \mid D} \frac{\log (p)}{p^{2}-1}\right]
\end{aligned}
$$

Now we return to the modified Eisenstein series

$$
\mathcal{E}(\tau, s ; D)=\mathbb{E}(\tau, s ; D)+\sum_{p \mid D} c_{p}(s) \mathbb{E}(\tau, s ; D / p)
$$

of (6.29) for $D=D(B)>1$. By (6.32), the Fourier coefficients of $\mathcal{E}^{\prime}\left(\tau, \frac{1}{2} ; D\right)$ for $m<0$ agree with those of $\mathbb{E}^{\prime}\left(\tau, \frac{1}{2} ; D\right)$, so that (8.28) gives part iii of Theorem 8.8.

If $m>0$ and for all $p \mid D, \chi_{d}(p) \neq 1$, note that by (8.12) and Lemma 8.5,

$$
\begin{aligned}
\mathbb{E}_{m}\left(\tau, \frac{1}{2} ; D / p\right) & =2 \delta(d ; D / p) H_{0}(m ; D / p) q^{m} \\
& =\left(1-\chi_{d}(p)\right)^{-1} \cdot \frac{1-\chi_{d}(p)+\chi_{d}(p) p^{k}-p^{k+1}}{1-p} \cdot 2 \delta(d ; D) H_{0}(m ; D) \cdot q^{m} \\
& =\left(1-\chi_{d}(p)\right)^{-1} \cdot \frac{1-\chi_{d}(p)+\chi_{d}(p) p^{k}-p^{k+1}}{1-p} \cdot \mathbb{E}_{m}\left(\tau, \frac{1}{2} ; D\right) .
\end{aligned}
$$

Therefore,

$$
\mathcal{E}_{m}^{\prime}\left(\tau, \frac{1}{2} ; D\right)=\mathbb{E}_{m}\left(\tau, \frac{1}{2} ; D\right)\left[\cdots+\sum_{p \mid D} c_{p}^{\prime}\left(\frac{1}{2}\right) \cdot\left(1-\chi_{d}(p)\right)^{-1} \cdot \frac{1-\chi_{d}(p)+\chi_{d}(p) p^{k}-p^{k+1}}{1-p}\right]
$$

where the dots indicate the expression in (8.24) for $\mathbb{E}_{m}^{\prime}\left(\tau, \frac{1}{2} ; D\right)$. For a prime $p \mid D$, we write $c^{\prime}$ for $c_{p}^{\prime}\left(\frac{1}{2}\right) / \log (p)$ and $k=\operatorname{ord}_{p}(n)$. Then, the coefficient of $\log (p)$ inside the brackets is

$$
-1-k-\frac{1}{\log (p)} \cdot \frac{b_{p}^{\prime}(n, 0 ; D)}{b_{p}(n, 0 ; D)}+c^{\prime} \cdot\left(1-\chi_{d}(p)\right)^{-1} \cdot \frac{1-\chi_{d}(p)+\chi_{d}(p) p^{k}-p^{k+1}}{1-p} .
$$




\section{S. S. Kudla, M. RAPOport And T. YAnG}

We now use Lemma 8.10, part ii. If $\chi_{d}(p)=-1,(8.33)$ gives

$$
\begin{aligned}
K_{p} & :=-1-k+\frac{2(p+1) p^{k+1}+p^{2}-4 p-1}{2\left(p^{2}-1\right)}+c^{\prime} \cdot \frac{1}{2} \cdot \frac{p^{k+1}+p^{k}-2}{p-1} \\
& =-1-k+\frac{1}{2}+\frac{p^{k+1}+p^{k}-2}{2(p-1)} \\
& =-k+\frac{(p+1)\left(p^{k}-1\right)}{2(p-1)} .
\end{aligned}
$$

If $\chi_{d}(p)=0,(8.33)$ gives

$$
\begin{aligned}
K_{p} & :=-1-k+\frac{2 p\left(p^{k+1}-1\right)}{p^{2}-1}+c^{\prime} \cdot \frac{p^{k+1}-1}{p-1} \\
& =-1-k+\frac{p^{k+1}-1}{p-1} .
\end{aligned}
$$

Thus, (8.26), (8.32) and these expressions for the coefficients $K_{p}$ of $\log (p)$ for $p \mid D$ yield part i of Theorem 8.8.

To prove part ii, suppose that $m>0$ and that there is a unique prime $p \mid D$ for which $\chi_{d}(p)=1$. Then, using (8.26) and (6.32), we have

$$
\begin{aligned}
\mathcal{E}_{m}^{\prime}\left(\tau, \frac{1}{2} ; D\right)= & \mathbb{E}_{m}^{\prime}\left(\tau, \frac{1}{2} ; D\right)+\sum_{\ell \mid D} c_{\ell}^{\prime}\left(\frac{1}{2}\right) \cdot \mathbb{E}_{m}\left(\tau, \frac{1}{2} ; D / \ell\right) \\
= & -2 \delta(d ; D / p) H_{0}(m ; D) \cdot q^{m} \cdot(p+1)^{-1} \cdot\left(1+p-2 p^{k+1}\right) \log (p) \\
& +c_{p}^{\prime}\left(\frac{1}{2}\right) \cdot 2 \delta(d ; D / p) H_{0}(m ; D / p) q^{m} \\
= & 2 \delta(d ; D / p) H_{0}(m ; D) \cdot q^{m} \cdot\left[-(p+1)^{-1} \cdot\left(1+p-2 p^{k+1}\right)+c^{\prime} \cdot p^{k}\right] \log (p) \\
= & 2 \delta(d ; D / p) H_{0}(m ; D) \cdot q^{m} \cdot\left(p^{k}-1\right) \log (p),
\end{aligned}
$$

as claimed.

Finally, we consider the constant term. By (8.30), Corollary 8.4 and (6.32), we have

$$
\begin{aligned}
\mathcal{E}_{0}^{\prime}(\tau, & \left.\frac{1}{2} ; D\right) \\
& =\mathbb{E}_{0}^{\prime}\left(\tau, \frac{1}{2} ; D\right)-\sum_{p \mid D} \frac{p-1}{p+1} \log (p) \cdot c(D / p) \Lambda_{D / p}(2) \\
& =c(D) \Lambda_{D}(2)\left[\frac{1}{2} \log (v)+1+\log (D)-\log (\pi)-\gamma+2 \frac{\zeta^{\prime}(2)}{\zeta(2)}+\sum_{p \mid D}\left(\frac{2}{p^{2}-1}+\frac{1}{p+1}\right) \log (p)\right] \\
& =c(D) \Lambda_{D}(2)\left[\frac{1}{2} \log (v)+1-\log (\pi)-\gamma+2 \frac{\zeta^{\prime}(2)}{\zeta(2)}+\sum_{p \mid D} \frac{p \log (p)}{p-1}\right] \\
& =c(D) \Lambda_{D}(2)\left[\frac{1}{2} \log (v)-2 \frac{\zeta^{\prime}(-1)}{\zeta(-1)}-1+2 \log (2)+\log (\pi)+\gamma+\sum_{p \mid D} \frac{p \log (p)}{p-1}\right] \\
& =c(D) \Lambda_{D}(2)\left[\frac{1}{2} \log (v)-2 \frac{\zeta^{\prime}(-1)}{\zeta(-1)}-1+2 C+\sum_{p \mid D} \frac{p \log (p)}{p-1}\right]
\end{aligned}
$$

where $C$ is as in Definition 3.4. Here we use the fact that

$$
c(D / p) \Lambda_{D / p}(2)=-c(D) \Lambda_{D}(2) \frac{p+1}{p^{2}-1} .
$$




\section{DeRivatives of Eisenstein SERIES AND FALtings Heights}

For later comparison, we note that the coefficient $A_{m}^{\prime}\left(\frac{1}{2}, v\right)$ in the term

$$
\mathcal{E}_{m}^{\prime}\left(\tau, \frac{1}{2} ; D\right)=A_{m}^{\prime}\left(\frac{1}{2}, v\right) q^{m}
$$

in part $\mathrm{i}$ of Theorem 8.8 can be written as a sum of four quantities:

$$
\begin{gathered}
2 \delta(d ; D) H_{0}(m ; D) \cdot\left[\frac{1}{2} \log (d)+\frac{L^{\prime}\left(1, \chi_{d}\right)}{L\left(1, \chi_{d}\right)}-\frac{1}{2} \log (\pi)-\frac{1}{2} \gamma\right], \\
2 \delta(d ; D) H_{0}(m ; D) \cdot \frac{1}{2} J(4 \pi m v), \\
2 \delta(d ; D) H_{0}(m ; D) \cdot \sum_{\substack{p \\
p \nmid D}}\left(\log |n|_{p}-\frac{b_{p}^{\prime}(n, 0 ; D)}{b_{p}(n, 0 ; D)}\right),
\end{gathered}
$$

and

$$
2 \delta(d ; D) H_{0}(m ; D) \cdot \sum_{\substack{p \\ p \mid D}} K_{p} \log (p) .
$$

\section{Part III. Computations: geometric}

\section{The geometry of $\mathcal{Z}(m)$}

In this section, we prepare the calculation of the coefficients of the generating series $\phi_{\operatorname{deg}}(\tau)$ and $\phi_{\text {height }}(\tau)$ by describing some of the geometry of the special cycles $\mathcal{Z}(m)$.

It turns out that the primes of bad reduction (i.e. $p \mid D(B)$ ) play a very special role. Namely,

$$
\mathcal{Z}(m) \times_{\operatorname{Spec} \mathbb{Z}} \operatorname{Spec} \mathbb{Z}\left[D(B)^{-1}\right] \text { is reduced and is finite and flat over } \operatorname{Spec} \mathbb{Z}\left[D(B)^{-1}\right] .
$$

We denote by $\mathcal{Z}(m)^{\text {horiz }}$ the closure of $\mathcal{Z}(m) \times_{\text {Spec } \mathbb{Z}} \operatorname{Spec} \mathbb{Z}\left[D(B)^{-1}\right]$ in $\mathcal{Z}(m)$ and call it the horizontal part of the special cycle.

We first describe the generic fiber of $\mathcal{Z}(m)$. As in $\S 5$, let $L=V \cap O_{B}$, let $L(m)$ be as in (5.7) and let

$$
D_{\mathcal{Z}(m)}=\coprod_{x \in L(m)} D_{x}
$$

as in (5.11). Then $\Gamma$ acts on $D_{\mathcal{Z}(m)}$ compatibly with its action on $D$, and we may represent $\mathcal{Z}(m)_{\mathbb{C}}$ as an orbifold mapping to $[\Gamma \backslash D]$ :

$$
\mathcal{Z}(m)_{\mathbb{C}}=\left[\Gamma \backslash D_{\mathcal{Z}(m)}\right] .
$$

We next give the degree of this orbifold. We recall the following notation from (8.2). For $x \in L$ with $Q(x)=m>0$, let

$$
k=\mathbb{Q}[x] \simeq \mathbb{Q}[X] /\left(X^{2}+m\right)=\mathbb{Q}(\sqrt{-m}) .
$$

Let $O_{k}$ be its ring of integers and let $-d=\operatorname{disc}\left(O_{k}\right)$ be its discriminant. Then the discriminant of the order $\mathbb{Z}[x]=\mathbb{Z}[X] /\left(X^{2}+m\right)$ is equal to $-4 m$. Write $4 m=n^{2} d$, as in (8.2). We note that there is a map of discrete orbifolds

$$
\left[\Gamma \backslash D_{\mathcal{Z}(m)}\right] \longrightarrow[\Gamma \backslash L(m)]
$$

which is 2 to 1 .

Proposition 9.1. For $m>0$ and $k$ as above:

i) if $k$ cannot be embedded into $B$, then $\mathcal{Z}(m)_{\mathbb{Q}}=\emptyset$;

ii) otherwise,

$$
\operatorname{deg} \mathcal{Z}(m)_{\mathbb{Q}}=2 \delta(d, D) H_{0}(m, D) .
$$




\section{S. S. Kudla, M. Rapoport And T. YAnG}

Here the degree of $\mathcal{Z}(m)_{\mathbb{Q}}$ is taken in the stack sense, i.e. each geometric point $\eta$ of $\mathcal{Z}(m)_{\mathbb{Q}}$ counts with multiplicity $1 /|\operatorname{Aut}(\eta)|$.

Proof.

i) For any $\mathbb{C}$-valued point $(A, \iota)$ of $\mathcal{M}$ we have an injection

$$
\operatorname{End}(A, \iota) \hookrightarrow B .
$$

Hence, if $(A, \iota, x)$ is a $\mathbb{C}$-valued point of $\mathcal{Z}(m)$ we obtain an injection $k=\mathbb{Q}[x] \hookrightarrow B$.

ii) Using (9.3), we obtain

$$
\operatorname{deg} \mathcal{Z}(m)_{\mathbb{Q}}=2 \sum_{\substack{x \in L(m) \\ \bmod \Gamma}} \frac{1}{\left|\Gamma_{x}\right|} .
$$

The following result finishes the proof of the proposition.

LEMMA 9.2. If $m>0$, then

$$
\sum_{\substack{x \in L(m) \\ \bmod \Gamma}} \frac{1}{\left|\Gamma_{x}\right|}=\delta(d ; D) \cdot H_{0}(m ; D),
$$

where $H_{0}(m ; D)$ and $\delta(d ; D)$ are given by $(8.17)$ and (8.18).

Proof. For any $x \in V(\mathbb{Q}) \cap O_{B}$ with $Q(x)=m$, there is an associated embedding $i_{x}: \mathbb{Q}(\sqrt{-m}) \rightarrow B$, taking $\sqrt{-m}$ to $x$. The order $O_{c^{2} d}=i_{x}^{-1}\left(O_{B}\right)$ is an invariant of the $\Gamma=O_{B}^{\times}$conjugacy class of $i_{x}$ and $i_{x}$ is an optimal embedding of $O_{c^{2}}$, in the terminology of Eichler [Eic56]. Recall that the order $\mathbb{Z}[\sqrt{-m}]$ has discriminant $-4 m$ and hence, conductor $n$.

Let

$$
\operatorname{Opt}\left(O_{c^{2} d}, O_{B}\right)=\left\{i: \mathbb{Q}(\sqrt{-m}) \rightarrow B \mid i^{-1}\left(O_{B}\right)=O_{c^{2} d}\right\} / \Gamma
$$

be the set of $\Gamma$ orbits of optimal embeddings. Recall that the order $i^{-1}\left(O_{B}\right)$ is maximal at all primes $p \mid D(B)$. The following fact is classical [Eic56]:

$$
\left|\operatorname{Opt}\left(O_{c^{2} d}, O_{B}\right)\right|=\delta(d ; D) \cdot h\left(c^{2} d\right) .
$$

Using (8.19), we have

$$
\begin{aligned}
\sum_{\substack{x \in L(m) \\
Q(x)=m \\
\bmod \Gamma}}\left|\Gamma_{x}\right|^{-1} & =\sum_{\substack{c \mid n \\
(c, D)=1}}\left|\operatorname{Opt}\left(O_{c^{2} d}, O_{B}\right)\right| \cdot\left|O_{c^{2} d}^{\times}\right|^{-1} \\
& =\delta(d ; D) \frac{h(d)}{w(d)} \sum_{\substack{c \mid n \\
(c, D)=1}} c \prod_{\ell \mid c}\left(1-\chi_{d}(\ell) \ell^{-1}\right) \\
& =\delta(d ; D) H_{0}(m ; D) .
\end{aligned}
$$

Proof of Proposition 7.1. Comparing the expression just found for $\operatorname{deg} \mathcal{Z}(m)_{\mathbb{Q}}$ with $(2.7)$, we have

$$
\phi_{\operatorname{deg}}(\tau)=\zeta_{D}(-1)+\sum_{m>0} 2 \delta(d ; D(B)) H_{0}(m ; D(B)) q^{m},
$$

which coincides with $\mathcal{E}\left(\tau, \frac{1}{2} ; D(B)\right)$, via Proposition 8.7, so Proposition 7.1 is proved. 


\section{Derivatives of Eisenstein series AND FAltings Heights}

Remark 9.3. The map $\mathcal{Z}(m)_{\mathbb{Q}} \rightarrow \mathcal{M}_{\mathbb{Q}}$ is not a closed immersion, hence $\mathcal{Z}(m)_{\mathbb{Q}}$ is not a divisor on $\mathcal{M}_{\mathbb{Q}}$. In fact, the morphism is of degree equal to 2 over its image. To see this, note that if $(A, \iota, x) \in \mathcal{Z}(m)(\mathbb{C})$, then $\operatorname{End}(A, \iota)_{\mathbb{Q}}=k=\mathbb{Q}(\sqrt{-m})$. Hence the only other point of $\mathcal{Z}(m)(\mathbb{C})$ mapping to $(A, \iota) \in \mathcal{M}(\mathbb{C})$ is $(A, \iota,-x)$. That the degree is 2 , even in the stack sense, follows from

$$
\operatorname{Aut}(A, \iota)=\operatorname{Aut}(A, \iota, x) \text {. }
$$

The stack $\mathcal{Z}(m)$ can have some pathological features in characteristic $p$ for $p \mid D(B)$. Namely, as already mentioned in $\S 5$, it can happen that $\mathcal{Z}(m)$ has dimension 0 (only if $k=\mathbb{Q}(\sqrt{-m}$ ) does not embed into $B$; cf. Proposition 9.1, part i) and also that $\mathcal{Z}(m)$ has embedded components. This leads us to introduce the Cohen-Macauleyfication $\mathcal{Z}(m)^{\text {pure }}[\mathrm{KR} 00 \mathrm{a}]$. In the case that $\mathcal{Z}(m)$ has dimension 0 , this is empty. In all other cases $\mathcal{Z}(m)$ may be considered as a divisor on $\mathcal{M}$ (but note that since $\mathcal{Z}(m)$ is not a closed substack of $\mathcal{M}$, the degree of $\mathcal{Z}(m)$ over its image must be taken into account). However, even after $\mathcal{Z}(m)$ is replaced by $\mathcal{Z}(m)^{\text {pure }}$, one interesting feature remains, namely the existence of vertical components in characteristic $p \mid D(B)$. We write

$$
\mathcal{Z}(m)^{\text {pure }}=\mathcal{Z}(m)^{\text {horiz }}+\mathcal{Z}(m)^{\text {vert }}
$$

(the equality of 'divisors' on $\mathcal{M}$ ), where $\mathcal{Z}(m)^{\text {vert }}$ is the sum with multiplicities of the irreducible vertical components in characteristic $p$ as $p$ runs over primes dividing $D(B)$. We note that if we redefine

$$
\hat{\mathcal{Z}}(m, v)=\left(\mathcal{Z}(m)^{\text {pure }}, \Xi(m, v)\right)
$$

then the expression $\langle\hat{\mathcal{Z}}(m, v), \hat{\omega}\rangle$ appearing in the definition of $\phi_{\text {height }}(\tau)$ remains unchanged (cf. [KR00a, § 4]). We remark in passing that if $\mathcal{Z}(m)_{\mathbb{Q}}=\emptyset$, then $\Xi(m, v)=0$; cf. (5.8).

Taking (9.10) into account and using formula (5.11) of Bost [Bos99], we may write

$$
\langle\hat{\mathcal{Z}}(m, v), \hat{\omega}\rangle=h_{\hat{\omega}}\left(\mathcal{Z}(m)^{\text {horiz }}\right)+h_{\hat{\omega}}\left(\mathcal{Z}(m)^{\text {vert }}\right)+\frac{1}{2} \int_{[\Gamma \backslash D]} \Xi(m, v) c_{1}(\hat{\omega}) .
$$

Also note that the second summand on the right-hand side may be written as the sum over contributions of the bad fibers,

$$
h_{\hat{\omega}}\left(\mathcal{Z}(m)^{\mathrm{vert}}\right)=\sum_{p \mid D(B)} h_{\hat{\omega}}\left(\mathcal{Z}(m)_{p}^{\mathrm{vert}}\right),
$$

where, since $\mathcal{Z}(m)^{\text {vert }}$ has empty generic fiber,

$$
h_{\hat{\omega}}\left(\mathcal{Z}(m)_{p}^{\text {vert }}\right)=\operatorname{deg}\left(\omega \mid \mathcal{Z}(m)_{p}^{\text {vert }}\right) \log (p) .
$$

Here $\mathcal{Z}(m)_{p}^{\text {vert }}=\mathcal{Z}(m)^{\text {vert }} \times_{\text {Spec } \mathbb{Z}} \operatorname{Spec} \mathbb{Z}_{p}$.

In the next three sections we will explicitly evaluate each summand on the right-hand side of (9.12).

\section{Contributions of horizontal components}

In this section, we compute the quantity $h_{\hat{\omega}}\left(\mathcal{Z}(m)^{\text {horiz }}\right)$. This is done in two steps. We first express this quantity in terms of the Faltings heights of certain abelian surfaces which are isogenous to products of CM-elliptic curves. We then determine the effect of the isogeny on the Faltings height.

Observe that $\mathcal{Z}(m)^{\text {horiz }}$ is a union of horizontal integral substacks

$$
\mathcal{Z}(m)^{\text {horiz }}=\sum_{\xi \in \mathcal{Z}(m)_{\mathbb{Q}}^{\text {horiz }}} \mathcal{Z}_{\xi},
$$

where $\xi$ is the generic point of $\mathcal{Z}_{\xi}$. Let $\tilde{\mathcal{Z}}_{\xi}$ be the normalization of $\mathcal{Z}_{\xi}$ and let $j_{\xi}: \tilde{\mathcal{Z}}_{\xi} \rightarrow \mathcal{M}$ be the composition of the normalization map $\widetilde{\mathcal{Z}}_{\xi} \rightarrow \mathcal{Z}_{\xi}$ with the morphism $\mathcal{Z}_{\xi} \rightarrow \mathcal{M}$. By linearity and 


\section{S. S. Kudla, M. Rapoport And T. YAnG}

the definition of $h_{\hat{\omega}}$ for horizontal cycles (cf. equation (4.4) and [Bos99]), we have

$$
\begin{aligned}
h_{\hat{\omega}}\left(\mathcal{Z}(m)^{\text {horiz }}\right) & =\sum_{\xi \in \mathcal{Z}(m)_{\mathbb{Q}}^{\text {horiz }}} h_{\hat{\omega}}\left(\mathcal{Z}_{\xi}\right) \\
& =\sum_{\xi \in \mathcal{Z}(m)_{\mathbb{Q}}^{\text {horiz }}} \widehat{\operatorname{deg}} j_{\xi}^{*} \hat{\omega} \cdot \frac{1}{|\operatorname{Aut}(\bar{\xi})|} \\
& =\frac{1}{|L: \mathbb{Q}|} \cdot \sum_{\eta \in \mathcal{Z}(m)^{\text {horiz }}(L)} \widehat{\operatorname{deg}} j_{\eta}^{*} \hat{\omega} \cdot \frac{1}{|\operatorname{Aut}(\eta)|},
\end{aligned}
$$

for any sufficiently large number field $L \subset \overline{\mathbb{Q}}$, where $\eta$ runs over the $L$ points of $\mathcal{Z}(m)$ and where the factors involving $\operatorname{Aut}(\bar{\xi})=\operatorname{Aut}\left((A, \iota, x)_{\bar{\xi}}\right)$ and $\operatorname{Aut}(\eta)=\operatorname{Aut}\left((A, \iota, x)_{\eta}\right)$ come in due to the stack. Here we also write $\eta$ for the extension to $\eta:$ Spec $O_{L} \rightarrow \mathcal{M}$. We may assume that $A_{\eta}$, the abelian variety over $L$ determined by $\eta$, has semistable reduction over $L$. Then, by definition, the Faltings height $h_{\mathrm{Fal}}^{*}\left(A_{\eta}\right)$ is given by

$$
h_{\mathrm{Fal}}^{*}\left(A_{\eta}\right)=|L: \mathbb{Q}|^{-1} \widehat{\operatorname{deg}} j_{\eta}^{*} \hat{\omega} .
$$

Here the notation $h_{\text {Fal }}^{*}$ indicates that we have used the metric $\|\cdot\|$ given in Definition 3.4, rather than the standard metric of (3.11). This point is discussed further below. Then:

$$
\begin{aligned}
& h_{\hat{\omega}}\left(\mathcal{Z}(m)^{\text {horiz }}\right)=\sum_{\eta \in \mathcal{Z}(m)^{\text {horiz }}(L)} h_{\text {Fal }}^{*}\left(A_{\eta}\right) \cdot \frac{1}{|\operatorname{Aut}(\eta)|} \\
& =2 \sum_{\substack{x \in L(m) \\
\bmod \Gamma}} h_{\mathrm{Fal}}^{*}\left(A_{x}\right) \cdot \frac{1}{\left|\Gamma_{x}\right|} .
\end{aligned}
$$

In the last expression, we have used the description of $\mathcal{Z}(m)(\mathbb{C})=\mathcal{Z}(m)^{\text {horiz }}(\mathbb{C})$ as the orbifold $\left[\Gamma \backslash D_{\mathcal{Z}(m)}\right]$, as in $\S 9$ where the map $\left[\Gamma \backslash D_{\mathcal{Z}(m)}\right] \rightarrow[\Gamma \backslash L(m)]$ is two to one, together with the fact that the abelian varieties associated to the two points in $D_{x}$ (i.e. having opposite complex structures) have the same Faltings height.

We next turn to the computation of the Faltings height $h_{\mathrm{Fal}}^{*}(A)$ of an abelian surface $A$ occurring in a triple $(A, \iota, x)$, where $\iota: O_{B} \rightarrow \operatorname{End}(A)$ and $x \in \operatorname{End}(A, \iota)$ is a special endomorphism with $Q(x)=m$, all defined over a number field $L$, where they all have good reduction. Let $\phi_{x}: k \rightarrow$ $\operatorname{End}^{0}(A)$ be the embedding determined by $\phi_{x}(\sqrt{-m})=x$ and let

$$
O_{c^{2} d}=\phi_{x}^{-1}(\mathbb{Q}[x] \cap \operatorname{End}(A)),
$$

where $O_{c^{2} d}$ is the order in $O_{k}$ of conductor $c$. In this case, we say that the triple $(A, \iota, x)$ is of type $c$. Recall that the order $\mathbb{Z}[\sqrt{-m}] \subset O_{c^{2} d} \subset O_{k}$ has discriminant $4 m$ and that we have written $4 m=n^{2} d$, where $-d$ is the discriminant of $O_{k}$. Then the order $\mathbb{Z}[\sqrt{-m}]$ has conductor $n$ and $c \mid n$. Note, in addition, that the order $O_{c^{2} d}$ must be maximal at all primes $p \mid D(B)$ and, hence, $(c, D(B))=1$.

Here the key point is that the special endomorphism $x$ with $x^{2}=-Q(x) \cdot 1_{A}$ forces $A$ to be isogenous to a product of elliptic curves with CM by $k=\mathbb{Q}(\sqrt{-m})$. The isogeny of interest is constructed as follows. Since $k$ splits $B$, we can choose an embedding of $\psi: k \hookrightarrow B$ such that

$$
O_{k}=\psi^{-1}\left(O_{B}\right),
$$

i.e. an optimal embedding, in the sense of Eichler. Then the endomorphisms

$$
\alpha_{x}^{ \pm}:=x \pm \iota \circ \psi(\sqrt{-m}) \in \operatorname{End}(A)
$$

satisfy

$$
\alpha_{x}^{+}+\alpha_{x}^{-}=2 x \text { and } \alpha_{x}^{+} \cdot \alpha_{x}^{-}=0 .
$$




\section{DeRivatives of Eisenstein SERIES AND FALtings Heights}

Let

$$
E^{ \pm}=\left(\operatorname{ker}\left(\alpha_{x}^{ \pm}\right)\right)^{0}
$$

be the identity component of the kernel of the endomorphism $\alpha_{x}^{ \pm}$. Choose an element $\eta \in O_{B}$ with $\operatorname{tr}(\eta)=0$ and such that conjugation by $\eta$ induces the Galois automorphism on $\psi(k)$. Note that

$$
\iota(\eta) \alpha_{x}^{+}=\alpha_{x}^{-} \iota(\eta)
$$

and so

$$
\iota(\eta) E^{ \pm}=E^{\mp} .
$$

Thus $E^{ \pm}$are both elliptic curves, and we obtain an isogeny

$$
u_{L}: E^{+} \times E^{-}=\left(\operatorname{ker}\left(\alpha_{x}^{+}\right)\right)^{0} \times\left(\operatorname{ker}\left(\alpha_{z}^{-}\right)\right)^{0} \longrightarrow A,
$$

rational over $L$. Moreover, the elliptic curves $E^{ \pm}=\left(\operatorname{ker}\left(\alpha_{x}^{ \pm}\right)\right)^{0}$ are stable under $\psi\left(O_{k}\right)$, i.e. they have complex multiplication by the full ring of integers of $k$. Note that the kernel of $u_{L}$ is the subgroup

$$
M_{L}=\left(\operatorname{ker}\left(\alpha_{x}^{+}\right)\right)^{0} \cap\left(\operatorname{ker}\left(\alpha_{x}^{-}\right)\right)^{0}
$$

embedded antidiagonally in $E^{+} \times E^{-}$.

The behavior of the Faltings height under isogeny is nicely described in the article of Raynaud [Ray85]. Our normalization of the Faltings height is the following. For an abelian variety $B$ of dimension $g$ over a number field $L$, let $N(B)$ be the Néron model of $B$ over $S=\operatorname{Spec} O_{L}$, and let $\omega_{B}=\epsilon^{*}\left(\wedge^{g} \Omega_{N(B) / S}\right)$ be the pullback by the zero section $\epsilon$ of the top power of the sheaf of relative differentials on $N(B)$. This invertible sheaf on $\operatorname{Spec} O_{L}$ has natural metrics; if $\sigma: L \hookrightarrow \mathbb{C}$ is an embedding of $L$, then a section $\beta$ of $\omega_{B}$ determines a holomorphic $g$-form on $B_{\sigma}(\mathbb{C})$, and ${ }^{4}$

$$
\|\beta\|_{\sigma, \text { nat }}^{2}=\left|\left(\frac{i}{2 \pi}\right)^{g} \int_{B_{\sigma}(\mathbb{C})} \beta \wedge \bar{\beta}\right| .
$$

If $B$ has semi-stable reduction, then the Faltings height of $B$ is given by

$$
h_{\mathrm{Fal}}(B)=|L: \mathbb{Q}|^{-1} \widehat{\operatorname{deg}}\left(\omega_{B}\right)
$$

where $\widehat{\operatorname{deg}}\left(\omega_{B}\right)$ denotes that Arakelov degree of $\omega_{B}$. The quantity $h_{\mathrm{Fal}}(B)$ does not depend on the choice of $L$ over which $B$ has semi-stable reduction.

In view of the normalization used in Definition 3.4, we introduce the metrics

$$
\|\beta\|_{\sigma}^{2}=\left|\left(e^{-C} \frac{i}{2 \pi}\right)^{g} \int_{B_{\sigma}(\mathbb{C})} \beta \wedge \bar{\beta}\right|
$$

where, as before, $C=\frac{1}{2}(\log (4 \pi)+\gamma)$. We denote the resulting height by $h_{\text {Fal }}^{*}(B)$. The two heights are related by

$$
h_{\mathrm{Fal}}^{*}(B)=h_{\mathrm{Fal}}(B)+\frac{1}{2} g C .
$$

Assume that $A$ has good reduction over $L$ and let $u_{L}: A \rightarrow B$ be an isogeny defined over $L$. Let $u: N(A) \rightarrow N(B)$ be the resulting homomorphism of Néron models with $M:=\operatorname{ker}(u)$. Then, as a special case of [Ray85, p. 205],

$$
h_{\mathrm{Fal}}(B)=h_{\mathrm{Fal}}(A)+\frac{1}{2} \log \left(\operatorname{deg}\left(u_{L}\right)\right)-|L: \mathbb{Q}|^{-1} \log \left|\epsilon^{*}\left(\Omega_{M / R}^{1}\right)\right| .
$$

The quantity $\delta(u):=\log \left|\epsilon^{*}\left(\Omega_{M / S}^{1}\right)\right|$ is a sum of local contributions as follows. For each prime $v$ of $L$ with $v \mid \operatorname{deg}\left(u_{L}\right)$, let $R_{v}$ be the completion of $O_{L}$ at $v$ and let $M_{v}=M \otimes_{O_{L}} R_{v}$. Then

$$
\delta(u)=\log \left|\epsilon^{*}\left(\Omega_{M / R}^{1}\right)\right|=\sum_{v \mid \operatorname{deg}\left(u_{L}\right)} \log \left|\epsilon^{*}\left(\Omega_{M_{v} / R_{v}}^{1}\right)\right| .
$$

${ }^{4}$ Note that we use the factor $(i / 2 \pi)^{g}$ rather than $(i / 2)^{g}$. This is the normalization used in Bost, [Bos99] for example. 


\section{S. S. Kudla, M. RAPOpORT AND T. YANG}

For convenience, we set

$$
\delta_{v}(u)=\log \left|\epsilon^{*}\left(\Omega_{M_{v} / R_{v}}^{1}\right)\right| .
$$

This quantity is invariant under base change in the sense that if $L^{\prime}$ is a finite extension of $L$ and if $u^{\prime}$ is the base change of $u$ to $\operatorname{Spec} O_{L^{\prime}}$, then

$$
\delta_{v}(u)=\sum_{w \mid v} \delta_{w}\left(u^{\prime}\right)
$$

where $w$ runs over the primes of $L^{\prime}$ dividing $v$.

We now return to our isogeny $u_{L}$, noting that $A$ and $E^{ \pm}$all have good reduction over $L$. Let

$$
u: N\left(E^{+}\right) \times N\left(E^{-}\right) \longrightarrow N(A)
$$

be the homomorphism induced by $u_{L}$ and let $M$ be its kernel. To calculate $\delta_{v}(u)$ for a prime $v \mid \operatorname{deg}\left(u_{L}\right)$, we pass to the $p$-divisible groups, where $p$ is the residue characteristic for $v$.

Let $G=G^{+} \times G^{-}$(respectively $A(p)$ ) be the $p$-divisible group over $R_{v}$ associated to $E^{+} \times E^{-}$ (respectively $A$ ) so that we have an exact sequence

$$
0 \longrightarrow C \longrightarrow G \longrightarrow A(p) \longrightarrow 0
$$

determined by the isogeny $u$. Since the prime to $p$ part of the kernel of $u$ is automatically étale over $R_{v}$, the invariant $\delta_{v}(u)$ depends only on the $p$-divisible groups and hence on $C$. The isogeny (10.23) corresponds to a submodule $T^{\prime}$ of $V(G)$, the rational Tate module of $G$,

$$
T(G) \subset T^{\prime} \subset V(G)=V\left(G^{+}\right) \oplus V\left(G^{-}\right) .
$$

The fact that $E^{ \pm}$maps injectively into $A$ implies that $G^{ \pm} \hookrightarrow A(p)$ and hence

$$
T^{\prime} \cap V\left(G^{ \pm}\right)=T\left(G^{ \pm}\right) .
$$

Thus there are isomorphisms

$$
\operatorname{pr}^{+}\left(T^{\prime}\right) / T\left(G^{+}\right) \stackrel{\sim}{\longleftarrow} T^{\prime} / T(G) \stackrel{\sim}{\longrightarrow} \operatorname{pr}^{-}\left(T^{\prime}\right) / T\left(G^{-}\right) .
$$

Proposition 10.1. Suppose that $p$ splits in $k$. Then $\operatorname{ord}_{p}\left(\operatorname{deg}\left(u_{L}\right)\right)=2 \operatorname{ord}_{p}(c)$. Moreover, for any place $v$ of $L$ with $v \mid p$, the group $C$ is étale over $R_{v}$, and hence

$$
\delta_{v}(u)=\log \left|\epsilon^{*}\left(\Omega_{M_{v} / R_{v}}^{1}\right)\right|=\log \left|\epsilon^{*}\left(\Omega_{C / R_{v}}^{1}\right)\right|=0 .
$$

Proof. We write

$$
k_{p} \simeq \mathbb{Q}_{p} \oplus \mathbb{Q}_{p}, \quad \alpha \mapsto\left(\alpha_{1}, \alpha_{2}\right),
$$

and let $\lambda_{1}$ and $\lambda_{2}$ be the corresponding algebra homomorphisms from $k_{p}$ to $\mathbb{Q}_{p}$. Let $G_{0}$ (respectively $\left.G_{\text {ét }}\right)$ be the connected (respectively étale) part of $G$, and note that, for example

$$
G_{0}=G_{0}^{+} \times G_{0}^{-} .
$$

Since the action of $\mathcal{O}:=O_{k} \otimes \mathbb{Z}_{p} \simeq \mathbb{Z}_{p} \oplus \mathbb{Z}_{p}$ preserves $G_{0}^{ \pm}$, the action of $\mathcal{O}$ on the Tate module $T\left(G^{ \pm}\right)$must have the form

$$
\begin{array}{ll}
T\left(G^{+}\right)=T\left(G_{0}^{+}\right) \oplus T\left(G_{\text {ét }}^{+}\right) \simeq \mathbb{Z}_{p} \oplus \mathbb{Z}_{p}, & \lambda_{1} \oplus \lambda_{2} \\
T\left(G^{-}\right)=T\left(G_{0}^{-}\right) \oplus T\left(G_{\text {ét }}^{-}\right) \simeq \mathbb{Z}_{p} \oplus \mathbb{Z}_{p}, & \lambda_{2} \oplus \lambda_{1},
\end{array}
$$

where the switch of characters is due to the fact that the isogeny induced by $\eta$ is $\mathcal{O}$-antilinear but must preserve the connected-étale decomposition. Thus there is a canonical decomposition

$$
T(G)=T\left(G_{0}^{+}\right) \oplus T\left(G_{0}^{-}\right) \oplus T\left(G_{\text {ét }}^{+}\right) \oplus T\left(G_{\text {ét }}^{-}\right), \quad \lambda_{1} \oplus \lambda_{2} \oplus \lambda_{2} \oplus \lambda_{1} .
$$

Since the $\mathbb{Z}_{p}$-lattice $T^{\prime} \subset V(G)$ is stable under $\mathcal{O}$, it must be generated by coset representatives of the form $(x, 0,0, w)$ and $(0, y, z, 0)$. Condition (10.24) implies, for example, that if $x=0$ for such a 


\section{DeRIVATIVES OF EISENSTEIN SERIES AND FAltings Heights}

representative, then $w=0$ (i.e. lies in $\mathbb{Z}_{p}$ ). It follows that

$$
T^{\prime}=\mathbb{Z}_{p} \cdot\left(p^{-r}, 0,0, \epsilon_{1} p^{-r}\right)+\mathbb{Z}_{p} \cdot\left(0, p^{-s}, \epsilon_{2} p^{-s}, 0\right)+T(G)
$$

for units $\epsilon_{1}$ and $\epsilon_{2}$ and non-negative integers $r$ and $s$. In addition, we can choose the element $\eta \in O_{B}$ above so that $\eta^{2}$ is prime to $p$ and so $\eta$ induces an automorphism on $A(p)$. Since $T^{\prime}$ is stable under this automorphism, we must have $r=s$ and $\epsilon_{1} \equiv \epsilon_{2} \bmod p^{r}$. On the other hand, the $\phi_{x^{-}}$action of $\mathcal{O}$ on $V(G)$ is given by

$$
\lambda_{2} \oplus \lambda_{2} \oplus \lambda_{1} \oplus \lambda_{1}
$$

The $\phi_{x}$ action of $\alpha \in \mathcal{O}$ preserves $T^{\prime}$ if and only if

$$
\alpha \in O_{c^{2} d} \otimes \mathbb{Z}_{p}=\left\{\left(a_{1}, a_{2}\right) \mathcal{O} \mid a_{1} \equiv a_{2} \bmod p^{\operatorname{ord}_{p}(c)}\right\} .
$$

Thus, we conclude that $r=\operatorname{ord}_{p}(c)$. This proves that $\operatorname{ord}_{p}\left(\operatorname{deg}\left(u_{L}\right)\right)=2 \operatorname{ord}_{p}(c)$.

To finish the proof of the Proposition, it suffices to show that

$$
T^{\prime} \cap V\left(G_{0}\right)=T\left(G_{0}\right),
$$

so that the projection to the étale part $G \rightarrow G_{\text {ét }}$ induces an isomorphism on $C$. However, this is clear from our description of the coset representatives.

Proposition 10.2. Suppose that $p$ is inert or ramified in $k$.

i) If $p \nmid D(B)$, then for any place $v$ of $L$ with $v \mid p$, there is a factorization $u=u^{\dagger} \circ u^{o}$ with isogenies $u^{\dagger}$ and $u^{o}$ such that

$$
\delta_{v}\left(u^{o}\right)=\frac{1}{2} \cdot\left|L_{v}: \mathbb{Q}_{p}\right| \cdot\left(\operatorname{ord}_{p}\left(\operatorname{deg}\left(u_{L}\right)\right)-2 \operatorname{ord}_{p}(c)\right) \cdot \log (p),
$$

and $\operatorname{ord}_{p}\left(\operatorname{deg}\left(u^{\dagger}\right)\right)=2 \operatorname{ord}_{p}(c)=2 s$. Moreover,

$$
\delta_{v}\left(u^{\dagger}\right)=\left|L_{v}: \mathbb{Q}_{p}\right| \cdot \frac{\left(1-p^{-s}\right) \cdot(1-\chi(p))}{\left(1-p^{-1}\right) \cdot(p-\chi(p))} \log (p) .
$$

Here $\chi(p)=-1$ if $p$ is inert and $\chi(p)=0$ if $p$ is ramified in $k$.

ii) If $p \mid D(B)$, then $\operatorname{ord}_{p}\left(\operatorname{deg}\left(u_{L}\right)\right)=0$ and $\delta_{v}(u)=0$.

Proof. Let $\mathbb{F}_{q}$ be the residue field $\mathcal{O} / \pi \mathcal{O}$, where $\pi$ is a fixed prime element of $\mathcal{O}$. Also write $\mathcal{O}_{s}=$ $O_{c^{2} d} \otimes \mathbb{Z}_{p}$, where $s=\operatorname{ord}_{p}(c)$. For convenience, we temporarily write $L$ in place of $L_{v}$ and $\mathcal{O}_{L}$ in place of $O_{L_{v}}$.

Now $G^{ \pm}=E^{ \pm}(p)$ is a formal group of dimension one and height two over $R_{v}=\mathcal{O}_{L_{v}}$ with an action of $\mathcal{O}$, i.e., a special formal $\mathcal{O}$-module in the sense of Drinfeld. We consider the sequence

$$
C \longrightarrow G^{+} \times G^{-} \stackrel{u}{\longrightarrow} A(p)
$$

and note that $O_{B} \otimes \mathcal{O}_{s}$ acts on $A(p)$. After replacing $L$ by a finite extension and using the invariance property (10.21), we may assume that $G_{0}:=G^{+} \simeq G^{-}$.

First suppose that $p \nmid D(B)$. Then, fixing an isomorphism

$$
O_{B} \otimes_{\mathbb{Z}} \mathcal{O}_{s} \simeq M_{2}\left(\mathbb{Z}_{p}\right) \otimes_{\mathbb{Z}_{p}} \mathcal{O}_{s} \simeq M_{2}\left(\mathcal{O}_{s}\right),
$$

we may write $A(p) \simeq G_{s} \times G_{s}$, where $G_{s}$ is a formal group of dimension 1 and height 2 . Since $(A, \iota, x)$ was supposed to be of type $c$, we have $\operatorname{End}\left(G_{s}\right)=\mathcal{O}_{s}$, hence the notation for $G_{s}$, consistent with that for $G_{0}$. The isogeny $u$ corresponds to an inclusion of Tate modules

$$
T\left(G_{0}\right) \oplus T\left(G_{0}\right)=T\left(G_{0} \times G_{0}\right) \subset T(A(p))=T\left(G_{s} \times G_{s}\right)=T\left(G_{s}\right) \oplus T\left(G_{s}\right) .
$$

Note that the two direct sums here are not necessarily compatible. Let $T\left(G_{s}\right)^{\dagger}$ be the largest $\mathcal{O}$-module contained in the $\mathcal{O}_{s}$-module $T\left(G_{s}\right)$. Note that

$$
T\left(G_{s}\right)^{\dagger} \oplus T\left(G_{s}\right)^{\dagger}
$$




\section{S. S. Kudla, M. RApoport And T. YAnG}

is the largest $\mathcal{O}$-module contained in $T\left(G_{s}\right) \oplus T\left(G_{s}\right)$. Hence, the inclusion (10.37) gives rise to a chain of inclusions

$$
T\left(G_{0}\right) \oplus T\left(G_{0}\right) \subset T\left(G_{s}\right)^{\dagger} \oplus T\left(G_{s}\right)^{\dagger} \subset T\left(G_{s}\right) \oplus T\left(G_{s}\right) .
$$

Hence, the isogeny $u$ factors as

$$
G_{0} \times G_{0} \stackrel{u^{o}}{\longrightarrow} G_{s}^{\dagger} \times G_{s}^{\dagger} \stackrel{u^{\dagger}}{\longrightarrow} G_{s} \times G_{s} .
$$

By the elementary divisor theorem, we can then find automorphisms $\alpha$ of $G_{0} \times G_{0}$ and $\beta$ of $G_{s}^{\dagger} \times G_{s}^{\dagger}$ such that $\beta \circ u^{o} \circ \alpha$ is of the form $u_{1}^{o} \times u_{2}^{o}$ for isogenies $u_{i}^{o}: G_{0} \rightarrow G_{s}^{\dagger}, i=1$, 2. Each of the isogenies $u_{i}^{o}$ corresponds to an inclusion of Tate modules $T\left(G_{0}\right) \subset T\left(G_{s}^{\dagger}\right) \subset V\left(G_{0}\right)$. Since both Tate modules are free $\mathcal{O}$-modules of rank 1 , there exists an isomorphism $G_{0} \simeq G_{s}^{\dagger}$ such that $u_{i}^{o}$ is given by multiplication by an element $\nu_{i} \in \mathcal{O}$. On the other hand, the isogeny $u^{\dagger}$ is of the form $u^{\dagger}=u_{1}^{\dagger} \times u_{1}^{\dagger}$, where the isogeny $u_{1}^{\dagger}$ is determined by the inclusion $T\left(G_{s}\right)^{\dagger} \subset T\left(G_{s}\right)$. If we choose an isomorphism $T\left(G_{s}\right)^{\dagger} \simeq \mathcal{O}$, then

$$
T\left(G_{s}\right) \simeq p^{-s} \epsilon \mathbb{Z}_{p}+\mathcal{O},
$$

where $\epsilon$ is a unit in $\mathcal{O}$. This implies that the degree of the isogeny $u_{1}^{\dagger}$ is $p^{s}$, as claimed.

The contribution $\delta_{v}\left(u^{\dagger}\right)=2 \delta_{v}\left(u_{1}^{\dagger}\right)$ of the isogeny $u^{\dagger}$ to the invariant $\delta_{v}(u)=\delta_{v}\left(u^{0}\right)+\delta_{v}\left(u^{\dagger}\right)$ can now be obtained from the following result, whose proof we include for the sake of completeness.

Proposition 10.3 (Nakkajima-Taguchi [NT91]). Let $k_{p} / \mathbb{Q}_{p}$ be a quadratic extension and let $L / k_{p}$

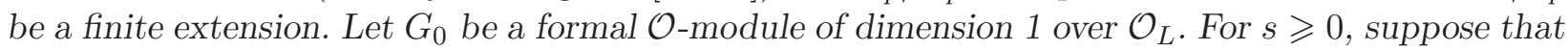
$\lambda: G_{0} \rightarrow G_{s}$ is an isogeny of degree $p^{s}$ over $\mathcal{O}_{L}$ such that $\operatorname{End}\left(G_{s}\right)=\mathcal{O}_{s}$. Let $D=\operatorname{Ker} \lambda$. Then

$$
\log \left|\varepsilon^{*} \Omega_{D / \mathcal{O}_{L}}^{1}\right|=\frac{1}{2}\left|L: \mathbb{Q}_{p}\right| \frac{\left(1-p^{-s}\right)(1-\chi(p))}{\left(1-p^{-1}\right)(p-\chi(p))} \log (p) .
$$

Here $\chi(p)$ is -1 (respectively 0) depending on whether $k_{p} / \mathbb{Q}_{p}$ is unramified or ramified.

Proof. (Sketch) Let $G_{0}$ be defined by the formal group law $g(X, Y) \in \mathcal{O}_{L}[[X, Y]]$. Then by Serre's isogeny formula [Gro86],

$$
D=\operatorname{Spec} \mathcal{O}_{L}[[X]] / \prod_{d \in D} g(X, d) .
$$

It follows that

$$
\begin{aligned}
\varepsilon^{*} \Omega_{D / \mathcal{O}_{L}}^{1} & =\mathcal{O}_{L} /\left(\prod_{d \in D} g(X, d)\right)_{X=0}^{\prime} \\
& =\mathcal{O}_{L} /\left(\prod_{d \in D \backslash\{0\}} d\right) .
\end{aligned}
$$

A consideration of the Newton polygon of $\left[\pi^{r}\right]_{G_{0}}=\pi^{r} X+\cdots$ shows that if $d \in D \backslash\{0\}$ is of precise $\pi$-order $r$, then

$$
\operatorname{ord}(d)=\frac{1}{q^{r}-q^{r-1}}
$$

Here, as before, $\pi$ denotes a prime element of $k_{p}$ and the ord function is normalized by $\operatorname{ord}(\pi)=1$. 


\section{DeRivatives of Eisenstein SERIES AND FALtings Heights}

It follows that

$$
\begin{aligned}
\lg _{\mathcal{O}_{L}}\left(\varepsilon^{*} \Omega_{D / \mathcal{O}_{L}}^{1}\right) & =e_{L / k_{p}} \cdot \sum_{d \in D \backslash\{0\}} \operatorname{ord}(d) \\
& =e_{L / k_{p}} \cdot \sum_{r=1}^{\infty} \frac{\ell(r)}{q^{r}-q^{r-1}} .
\end{aligned}
$$

Here $\ell(r)$ denotes the number of elements of $D(\bar{L})$ of exact $\pi$-order $r$. However, the conditions $\operatorname{deg}(\lambda)=p^{s}$ and $\operatorname{End}\left(G_{s}\right)=\mathcal{O}_{s}$ imply that

$$
D(\bar{L}) \cong T\left(G_{s}\right) / T\left(G_{0}\right) \cong\left(p^{-s} \varepsilon \mathbb{Z}_{p}+\mathcal{O}\right) / \mathcal{O} \cong p^{-s} \mathbb{Z}_{p} / \mathbb{Z}_{p},
$$

where $\varepsilon$ is a unit in $\mathcal{O}$. Hence, for $r \geqslant 1$,

$$
\ell(r)= \begin{cases}p^{r}-p^{r-1}, \quad 1 \leqslant r \leqslant s, & \text { if } k_{p} / \mathbb{Q}_{p} \text { is unramified } \\ p^{r / 2}-p^{r / 2-1}, \quad 1 \leqslant r \leqslant 2 s, 2 \mid r, & \text { if } k_{p} / \mathbb{Q}_{p} \text { is ramified }\end{cases}
$$

and is zero in all other cases. It follows that

$$
\begin{aligned}
\log \left|\varepsilon^{*} \Omega_{D / \mathcal{O}_{L}}^{1}\right| & =f_{L / \mathbb{Q}_{p}} \cdot \lg _{\mathcal{O}_{L}}\left(\varepsilon^{*} \Omega_{D / \mathcal{O}_{L}}^{1}\right) \cdot \log (p) \\
& =f_{L / \mathbb{Q}_{p}} \cdot e_{L / k_{p}} \cdot \sum_{r=1}^{s} \frac{\ell(r)}{q^{r}-q^{r-1}} \cdot \log (p),
\end{aligned}
$$

which yields the assertion.

Finally, the contribution $\delta_{v}\left(u^{o}\right)=\delta_{v}\left(u_{1}^{o}\right)+\delta_{v}\left(u_{2}^{o}\right)$ is given by the following result.

Lemma 10.4. For extensions $L / k_{p} / \mathbb{Q}_{p}$ as in the previous proposition, suppose that $G_{0}$ is a formal $\mathcal{O}$-module of dimension 1 over $\mathcal{O}_{L}$. Let $\lambda: G_{0} \rightarrow G_{0}$ be the isogeny given by multiplication by a non-zero element $\nu \in \mathcal{O}$. Let $D=\operatorname{ker}(\lambda)$. Then

$$
\log \left|\varepsilon^{*} \Omega_{D / \mathcal{O}_{L}}^{1}\right|=\frac{1}{2}\left|L: \mathbb{Q}_{p}\right| \cdot \log (\operatorname{deg} \lambda) .
$$

Proof. Let $s=\operatorname{ord}(\nu)$. Then, there are $q^{r}-q^{r-1}$ elements in $D(\bar{L}) \simeq \pi^{-s} \mathcal{O} / \mathcal{O}$ of exact order $\pi^{r}$, for $1 \leqslant r \leqslant s$, and none for all other $r$. Hence

$$
\lg _{\mathcal{O}_{L}}\left(\varepsilon^{*} \Omega_{D / \mathcal{O}_{L}}^{1}\right)=e_{L / k_{p}} \cdot \sum_{r=1}^{s} \frac{q^{r}-q^{r-1}}{q^{r}-q^{r-1}}=e_{L / k_{p}} \cdot s .
$$

It follows that

$$
\log \left|\varepsilon^{*} \Omega_{D / O_{L}}^{1}\right|=e_{L / k_{p}} \cdot f_{L / \mathbb{Q}_{p}} \cdot s \cdot \log (p)=\frac{1}{2}\left|L: \mathbb{Q}_{p}\right| \cdot s \cdot \log (q) .
$$

The assertion follows since the isogeny $\lambda$ has degree $q^{s}$.

Finally, we consider the case $p \mid D(B)$, and we recall that $\operatorname{ord}_{p}(c)=0$, so that $\mathcal{O}_{s}=\mathcal{O}_{0}=\mathcal{O}$. Once again, we consider the action of $O_{B} \otimes \mathcal{O}$ on $A(p)$. We fix an isomorphism

$$
B \otimes k_{p} \simeq M_{2}\left(k_{p}\right), \quad \text { with } O_{B} \otimes \mathcal{O} \hookrightarrow M_{2}(\mathcal{O})
$$

and such that, for $\alpha \in k_{p}$,

$$
1 \otimes \alpha \mapsto\left(\begin{array}{cc}
\alpha & \\
& \alpha
\end{array}\right) \quad \text { and } \quad \psi(\alpha) \otimes 1 \mapsto\left(\begin{array}{cc}
\alpha & \\
& \alpha^{\sigma}
\end{array}\right),
$$

where $\psi$ is the embedding of $k$ into $B$ chosen above. 


\section{S. S. Kudla, M. Rapoport And T. YAng}

LEMMA 10.5.

i) If $k_{p} / \mathbb{Q}_{p}$ is unramified, then the image of $O_{B} \otimes \mathcal{O}$ in $M_{2}(\mathcal{O})$ is the order

$$
\left(\begin{array}{cc}
\mathcal{O} & \mathcal{O} \\
p \mathcal{O} & \mathcal{O}
\end{array}\right)
$$

ii) If $k_{p} / \mathbb{Q}_{p}$ is ramified, then there is an element $\lambda \in\left(O_{B} \otimes \mathcal{O}\right)^{\times}$whose image in $G L_{2}\left(\mathbb{F}_{p}\right)$ under the composition of maps

$$
\mathrm{O}_{B} \otimes \mathcal{O} \longrightarrow M_{2}(\mathcal{O}) \longrightarrow M_{2}\left(\mathbb{F}_{p}\right)
$$

has eigenvalues which are not rational over $\mathbb{F}_{p}$.

iii) In the ramified case, suppose that $\Lambda$ is an $\mathcal{O}$-lattice contained in $k^{2}$ which is stable under $O_{B} \otimes \mathcal{O}$. Then $\Lambda$ is homothetic to $\Lambda_{0}=\mathcal{O}^{2}$.

Remark 10.6. In fact, if $p \neq 2$ and $k_{p} / \mathbb{Q}_{p}$ is ramified, then the image of $O_{B} \otimes \mathcal{O}$ in $M_{2}(\mathcal{O})$ is conjugate to $\operatorname{red}^{-1}\left(\mathbb{F}_{p^{2}}\right)$, where

$$
\text { red : } M_{2}(\mathcal{O}) \longrightarrow M_{2}\left(\mathbb{F}_{p}\right)
$$

is the reduction modulo $\pi$, and $\mathbb{F}_{p^{2}}$ is the non-trivial quadratic extension of $\mathbb{F}_{p}$, viewed as a subalgebra of $M_{2}\left(\mathbb{F}_{p}\right)$.

Proof. In the unramified case, we may write

$$
O_{B} \otimes \mathbb{Z}_{p}=\mathcal{O}\langle\Pi\rangle
$$

where $\Pi \in O_{B}$ is an element with $\Pi^{2}=p$ which normalizes $\psi\left(k_{p}\right)$ and acts on it by the Galois automorphism $\sigma$, i.e. $\Pi \alpha=\alpha^{\sigma} \Pi$. The image of $O_{B} \otimes \mathcal{O}$ in $M_{2}(\mathcal{O})$ is then generated by the elements of the form

$$
\left(\begin{array}{ll}
\alpha & \\
& \alpha
\end{array}\right), \quad\left(\begin{array}{ll}
\alpha & \\
& \alpha^{\sigma}
\end{array}\right) \text { and }\left(\begin{array}{cc}
1 \\
p &
\end{array}\right),
$$

for $\alpha \in \mathcal{O}$. Since $k_{p}$ is unramified, there is an $\alpha \in \mathcal{O}$ such that $\alpha-\alpha^{\sigma}$ is a unit, and hence such elements generate the Eichler order as claimed in part i.

Next suppose that $k_{p}$ is ramified. Let $k_{o}$ be the unramified quadratic extension of $\mathbb{Q}_{p}$ and let $\mathcal{O}_{o}$ be its ring of integers, with generator $\lambda$ having unit norm. Again, we can write $O_{B} \otimes \mathbb{Z}_{p}=\mathcal{O}_{o}\langle\Pi\rangle$. The image of $\lambda \otimes 1 \in\left(O_{B} \otimes \mathcal{O}\right)^{\times}$now gives the element required in part ii.

Finally, to prove part iii, observe that the lattice $\Lambda_{0}=\mathcal{O}^{2}$ is preserved by $O_{B} \otimes \mathcal{O}$ and, hence, is fixed by the element $\lambda$ of part ii. If $\Lambda$ is another $\mathcal{O}$-lattice, preserved by $O_{B} \otimes \mathcal{O}$, then $\Lambda$ must also be fixed by $\lambda$. The whole geodesic joining the vertices $\left[\Lambda_{0}\right]$ and $[\Lambda]$ in the building of $\mathrm{PGL}_{2}\left(k_{p}\right)$ is then fixed by $\lambda$. In particular, $\lambda$ must then fix a vertex at distance equal to 1 from $\left[\Lambda_{0}\right]$. However, this implies that the image of $\lambda$ in $\mathrm{PGL}_{2}\left(\mathbb{F}_{p}\right)$ has a fixed point on $\mathbb{P}^{1}\left(\mathbb{F}_{p}\right)=\mathbb{P}\left(\Lambda_{0} / \pi \Lambda_{0}\right)$ and hence an $\mathbb{F}_{p}$-rational eigenvector/eigenvalue, which has been excluded.

Returning to $A(p)$ and our isogeny, the isomorphism (10.51) determines an isomorphism

$$
V(A(p)) \simeq k^{2}
$$

under which

$$
V\left(G^{+}\right)=k \cdot\left(\begin{array}{l}
0 \\
1
\end{array}\right) \text { and } V\left(G^{-}\right)=k \cdot\left(\begin{array}{l}
0 \\
1
\end{array}\right) .
$$

The image of the Tate module $T(A(p))$ in $k^{2}$ is an $\mathcal{O}$-lattice which is stable under the action of $O_{B} \otimes \mathcal{O}$. 


\section{DeRivatives of Eisenstein SERIES AND FALtings Heights}

If $k_{p}$ is unramified, then $T(A(p)) \subset k^{2}$ is an $\mathcal{O}$-lattice stable under the Eichler order $O^{\prime} \subset M_{2}(\mathcal{O})$ in part $\mathrm{i}$ of Lemma 10.5. Let $1_{2}=e_{+}+e_{-} \in O^{\prime}$ where

$$
e_{+}=\left(\begin{array}{ll}
0 & 0 \\
0 & 1
\end{array}\right) \quad \text { and } \quad e_{-}=\left(\begin{array}{ll}
1 & 0 \\
0 & 0
\end{array}\right) \in O^{\prime}
$$

If $y=y_{+}+y_{-} \in T(A(p))$ with $y_{ \pm} \in V\left(G^{ \pm}\right)$, then $y_{ \pm}=e_{ \pm} y \in V\left(G^{ \pm}\right) \cap T(A(p))=T\left(G^{ \pm}\right)$and, hence, $T(A(p))=T\left(G^{+} \times G^{-}\right)$. Thus, our isogeny has degree 1 and $\delta_{v}(u)=0$.

If $k_{p} / \mathbb{Q}_{p}$ is ramified, then, by part iii of Lemma $10.5, T(A(p))$ is homothetic to $\Lambda_{0}=\mathcal{O}^{2}$. However, since $T\left(G^{ \pm}\right)=T(A(p)) \cap V\left(G^{ \pm}\right)$, we then simply have $T\left(G^{+} \times G^{-}\right)=T(A(p))$, so again our isogeny has degree 1 and $\delta_{v}(u)=0$. This finishes the proof of part ii of Proposition 10.2.

We can now compute the Faltings height.

Theorem 10.7. Suppose that the triple $(A, \iota, x)$, defined over a number field $L$, is of type $c$. Write $4 m=n^{2} d$, with $-d$ a fundamental discriminant and let $E=E_{d}$ be an elliptic curve over $L$ with complex multiplication by $O_{k}$, the ring of integers in $k=\mathbb{Q}(\sqrt{-d})$. Then

$$
h_{\mathrm{Fal}}^{*}(A)=2 h_{\mathrm{Fal}}^{*}(E)+\log (c)-\sum_{p} \frac{\left(1-p^{-\operatorname{ord}_{p}(c)}\right) \cdot(1-\chi(p))}{\left(1-p^{-1}\right) \cdot(p-\chi(p))} \cdot \log (p) .
$$

Here $\chi=\chi_{d}$ is as in (8.3).

Proof. We apply formula (10.18) to the isogeny $E^{+} \times E^{-} \rightarrow A$ defined above. The change in the Faltings height due to the isogeny has the form

$$
\frac{1}{2} \log \left(\operatorname{deg}\left(u_{L}\right)\right)-\frac{1}{|L: \mathbb{Q}|} \sum_{v} \delta_{v}(u) .
$$

We write

$$
\frac{1}{2} \log \left(\operatorname{deg}\left(u_{L}\right)\right)=\sum_{p} \frac{1}{2} \operatorname{ord}_{p}\left(\operatorname{deg}\left(u_{L}\right)\right) \cdot \log (p)
$$

so that $(10.58)$ can be written as a sum of local contributions $(10.58)_{p}$ which we now describe case by case.

If $p$ is split in $k$, then by Proposition 10.1,

$$
(10.58)_{p}=\operatorname{ord}_{p}(c) \cdot \log (p) .
$$

If $p \nmid D(B)$ is inert or ramified, by Proposition $10.2,(10.58)_{p}$ is equal to:

$$
\begin{aligned}
\frac{1}{2} \operatorname{ord}_{p}\left(\operatorname{deg}\left(u_{L}\right)\right) \cdot \log (p)-\frac{1}{|L: \mathbb{Q}|} \sum_{v \mid p}\left|L_{v}: \mathbb{Q}_{p}\right| \\
\quad \times\left(\frac{1}{2}\left[\operatorname{ord}_{p}\left(\operatorname{deg}\left(u_{L}\right)\right)-2 r\right]+\frac{\left(1-p^{-r}\right) \cdot(1-\chi(p))}{\left(1-p^{-1}\right) \cdot(p-\chi(p))}\right) \log (p) \\
=\left(r-\frac{\left(1-p^{-r}\right)(1-\chi(p))}{\left(1-p^{-1}\right)(p-\chi(p))}\right) \log (p),
\end{aligned}
$$

where we have set $r=\operatorname{ord}_{p}(c)$.

If $p \mid D(B)$, then by the considerations after Lemma $10.5,(10.58)_{p}=0$. 


\section{S. S. Kudla, M. Rapoport And T. YAnG}

THEOREM 10.8. The contribution of the 'horizontal' part to the pairing $\langle\hat{\mathcal{Z}}(m, v), \hat{\omega}\rangle$ is

$$
\begin{aligned}
h_{\hat{\omega}}\left(\mathcal{Z}(m)^{\text {horiz }}\right)= & 2 \delta(d, D) H_{0}(m, D(B)) 2 h_{\mathrm{Fal}}^{*}(E) \\
& +2 \delta(d, D) \frac{h(d)}{w(d)} \sum_{\substack{c \mid n \\
(c, D(B))=1}} c \prod_{\ell \mid c}\left(1-\chi(\ell) \ell^{-1}\right) \cdot \sum_{p} \eta_{p}\left(\operatorname{ord}_{p}(c)\right) \log (p),
\end{aligned}
$$

where, for $r \in \mathbb{Z}_{\geqslant 0}$,

$$
\eta_{p}(r)=r-\frac{\left(1-p^{-r}\right)(1-\chi(p))}{\left(1-p^{-1}\right)(p-\chi(p))} .
$$

Proof. Continuing (10.4) above, we have

$$
\begin{aligned}
& h_{\hat{\omega}}\left(\mathcal{Z}(m)^{\text {horiz }}\right)=2 \sum_{c \mid n} \sum_{\substack{x \in L(m) \\
\text { mod } \Gamma \\
\text { type } c}} h_{\text {Fal }}^{*}\left(A_{x}\right) \cdot \frac{1}{\left|\Gamma_{x}\right|} \\
& =2 \sum_{c \mid n}\left(\sum_{\substack{x \in L(m) \\
\text { mod } \Gamma \\
\text { type } c}} \frac{1}{\left|\Gamma_{x}\right|}\right)\left(2 h_{\text {Fal }}^{*}(E)+\log (c)-\sum_{p} \frac{\left(1-p^{-\operatorname{ord}_{p}(c)}\right) \cdot(1-\chi(p))}{\left(1-p^{-1}\right) \cdot(p-\chi(p))} \log (p)\right) \\
& =2 \delta(d, D) H_{0}(m, D(B)) 2 h_{\mathrm{Fal}}^{*}(E) \\
& +2 \delta(d, D) \frac{h(d)}{w(d)} \sum_{\substack{c \mid n \\
(c, D(B))=1}} c \prod_{\ell \mid c}\left(1-\chi(\ell) \ell^{-1}\right) \cdot \sum_{p} \eta_{p}\left(\operatorname{ord}_{p}(c)\right) \log (p),
\end{aligned}
$$

as claimed.

We now make the comparison of this expression with terms arising in the positive Fourier coefficients of the derivative of the modified Eisenstein series. To do this, we need a better expression for the sum on $c$ in the second term in Theorem 10.8. For convenience in the calculations, we let

$$
\beta_{p}(k)=-2 k+ \begin{cases}\frac{p^{k}-1}{p^{k}(p-1)}, & \text { if } \chi_{d}(p)=1, \\ \frac{(3 p+1)\left(p^{k}-1\right)-4 k(p-1)}{(p-1)\left(p^{k+1}+p^{k}-2\right)}, & \text { if } \chi_{d}(p)=-1, \\ \frac{2}{p-1}-\frac{2 k+2}{p^{k+1}-1}, & \text { if } \chi_{d}(p)=0 .\end{cases}
$$

Note that when $k=\operatorname{ord}_{p}(n)$, then, by part i of Lemma 8.10,

$$
\beta_{p}(k)=\frac{1}{\log (p)} \cdot \frac{b_{p}^{\prime}(n, 0 ; D)}{b_{p}(n, 0 ; D)} .
$$

Lemma 10.9. Let $4 m=n^{2} d$, as before. Then the following identity holds for any square-free $D>0$ :

$$
\begin{aligned}
& \frac{h(d)}{w(d)} \sum_{\substack{c \mid n \\
(c, D)=1}} c \prod_{p \mid c}\left(1-\chi_{d}(p) p^{-1}\right) \sum_{p \mid c} \eta_{p}\left(\operatorname{ord}_{p}(c)\right) \log (p) \\
&=H_{0}(m ; D) \cdot \sum_{\substack{p \\
(p, D)=1}}\left(-\operatorname{ord}_{p}(n)-\beta_{p}\left(\operatorname{ord}_{p}(n)\right)\right) \log (p) \\
&=H_{0}(m ; D) \cdot \sum_{\substack{p \\
(p, D)=1}}\left(\log |n|_{p}-\frac{b_{p}^{\prime}(n, 0 ; D)}{b_{p}(n, 0 ; D)}\right) .
\end{aligned}
$$




\section{DeRivatives of Eisenstein SERIES AND FALtings Heights}

Proof. We note that the sum in the last expression of the lemma (right-hand side) is finite since only summands for $p$ with $p \mid n$ are non-zero. We proceed by induction on the number of prime factors of $n$. To start the induction, let $n=p^{t}$. Then the first expression of the lemma (left-hand side) is equal to

$$
\frac{h(d)}{w(d)} \cdot \sum_{r=1}^{t} p^{r}\left(1-\chi_{d}(p) p^{-1}\right) \eta_{p}(r) \cdot \log (p)
$$

(note that the contribution of $c=1$ is trivial). By (8.17) and (10.62), the right-hand side is equal to

$$
\frac{h(d)}{w(d)} \cdot\left(\sum_{r=1}^{t} p^{r}\left(1-\chi_{d}(p) p^{-1}\right)+1\right) \cdot\left(-t-\beta_{p}(t)\right) \cdot \log (p) .
$$

Case by case, one can check that these two expressions coincide.

Case $\chi_{d}(p)=1$. Then $(10.64)$ without the factor $h(d) / w(d) \cdot \log (p)$ is equal to

$$
\sum_{r=1}^{t} p^{r}\left(1-p^{-1}\right) r=(p-1) \sum_{r=1}^{t} r p^{r-1}=t p^{t}-\frac{p^{t}-1}{p-1} .
$$

On the other hand, (10.65) without the factor $h(d) / w(d) \cdot \log (p)$ is equal to

$$
\left(\sum_{r=1}^{t} p^{r}\left(1-p^{-1}\right)+1\right) \cdot\left(t-p^{-t} \frac{p^{t}-1}{p-1}\right)=p^{t}\left(t-p^{-t} \frac{p^{t}-1}{p-1}\right) .
$$

Case $\chi_{d}(p)=-1$. Then (10.64) without the factor $h(d) / w(d) \cdot \log (p)$ is equal to

$$
\begin{aligned}
\sum_{r=1}^{t} p^{r}\left(1+p^{-1}\right)\left(r-2 p^{-r+1} \frac{p^{r}-1}{p^{2}-1}\right) & =\left(1+p^{-1}\right) \sum_{r=1}^{t}\left(r p^{r}-2 p \frac{p^{r}-1}{p^{2}-1}\right) \\
& =\frac{(p-1) t\left(p^{t}(p+1)+2\right)-(3 p+1)\left(p^{t}-1\right)}{(p-1)^{2}} .
\end{aligned}
$$

On the other hand, (10.65) without the factor $h(d) / w(d) \cdot \log (p)$ is equal to

$$
\begin{gathered}
\left(\sum_{r=1}^{t} p^{r}\left(1+p^{-1}\right)+1\right)\left(t-\frac{(3 p+1)\left(p^{t}-1\right)-4 t(p-1)}{(p-1)\left(p^{t+1}+p^{t}-2\right)}\right) \\
=\frac{p^{t+1}+p^{t}-2}{p-1}\left(t-\frac{(3 p+1)\left(p^{t}-1\right)-4 t(p-1)}{(p-1)\left(p^{t+1}+p^{t}-2\right)}\right) \\
=\frac{(p-1) t\left(p^{t}(p+1)+2\right)-(3 p+1)\left(p^{t}-1\right)}{(p-1)^{2}} .
\end{gathered}
$$

Case $\chi_{d}(p)=0$. Then (10.64) without the factor $h(d) / w(d) \cdot \log (p)$ is equal to

$$
\begin{aligned}
\sum_{r=1}^{t} p^{r}\left(r-p^{-r} \frac{p^{r}-1}{p-1}\right) & =\sum_{r=1}^{t} r p^{r}-\sum_{r=1}^{t} \frac{p^{r}-1}{p-1} \\
& =\frac{p^{t+1}-1}{p-1}\left(t-\frac{2}{p-1}+\frac{2 t+2}{p^{t+1}-1}\right) .
\end{aligned}
$$

On the other hand, (10.65) without the factor $h(d) / w(d) \cdot \log (p)$ is equal to

$$
\left(\sum_{r=0}^{t} p^{r}\right)\left(t-\frac{2}{p-1}+\frac{2 t+2}{p^{t+1}-1}\right)=\frac{p^{t+1}-1}{p-1}\left(t-\frac{2}{p-1}+\frac{2 t+2}{p^{t+1}-1}\right) .
$$




\section{S. S. Kudla, M. RAPOPORT AND T. YANG}

We therefore have checked the beginning of the induction. Let us now perform the induction step. Let $n=p^{t} \cdot n_{0}$, where $p \nmid n_{0}$. Let us put $m_{0}=m / p^{2 t}$, so that $4 m_{0}=n_{0}^{2} d$. We may assume that $p \nmid D$ because otherwise both sides of the identity for $n$ coincide with the corresponding sides of the identity for $n_{0}$, so that we may apply the induction hypothesis. We write $\mathcal{L}(m)$ (respectively $\mathcal{R}(m)$ ) for the left-hand side (respectively right-hand side) of our identity corresponding to $m$. Then $\mathcal{L}(m)$ is equal to

$$
\begin{aligned}
& \frac{h(d)}{w(d)} \sum_{r=1}^{t} p^{r}\left(1-\chi_{d}(p) p^{-1}\right) \sum_{\substack{c_{0} \mid n_{0} \\
\left(c_{0}, D\right)=1}} c_{0} \cdot\left(\prod_{\ell \mid c_{0}}\left(1-\chi_{d}(\ell) \ell^{-1}\right)\right)\left[\eta_{p}(r) \log (p)+\sum_{\ell \mid c_{0}} \eta_{\ell}\left(r_{\ell}\left(c_{0}\right)\right) \log (\ell)\right] \\
&+\frac{h(d)}{w(d)} \sum_{\substack{c_{0} \mid n_{0} \\
\left(c_{0}, D\right)=1}} c_{0} \cdot\left(\prod_{\ell \mid c_{0}}\left(1-\chi_{d}(\ell) \ell^{-1}\right)\right) \cdot \sum_{\ell \mid c_{0}} \eta_{\ell}\left(r_{\ell}\left(c_{0}\right)\right) \log (\ell) . \quad(10.72)
\end{aligned}
$$

We recall that

$$
\frac{h(d)}{w(d)} \cdot \sum_{\substack{c_{0} \mid n_{0} \\\left(c_{0}, D\right)=1}} c_{0} \prod_{\ell \mid c_{0}}\left(1-\chi_{d}(\ell) \ell^{-1}\right)=H_{0}\left(m_{0} ; D\right)
$$

Hence, we can write the above expression as a sum of three terms, the first one being

$$
\begin{gathered}
\frac{h(d)}{w(d)} \sum_{\substack{c_{0} \mid n_{0} \\
\left(c_{0}, D\right)=1}} c_{0}\left(\prod_{\ell \mid c_{0}}\left(1-\chi_{d}(\ell) \ell^{-1}\right)\right) \cdot\left(1-\chi_{d}(p) p^{-1}\right) \sum_{r=1}^{t} p^{r} \eta_{p}(r) \log (p) \\
=H_{0}\left(m_{0}, D\right) \cdot\left(1-\chi_{d}(p) p^{-1}\right) \sum_{r=1}^{t} p^{r} \eta_{p}(r) \log (p) .
\end{gathered}
$$

The second and the third term are, respectively, equal to

$$
\mathcal{L}\left(m_{0}\right) \cdot\left(1-\chi_{d}(p) \cdot p^{-1}\right) \sum_{r=1}^{t} p^{r}=\mathcal{L}\left(m_{0}\right) \cdot\left(1-\chi_{d}(p) p^{-1}\right) p \frac{p^{t}-1}{p-1}
$$

and $\mathcal{L}\left(m_{0}\right)$.

We thus obtain

$$
\mathcal{L}(m)=\frac{p^{t+1}-\chi_{d}(p) p^{t}+\chi_{d}(p)-1}{p-1} \cdot \mathcal{L}\left(m_{0}\right)+H_{0}\left(m_{0}, D\right) \cdot\left(1-\chi_{d}(p) p^{-1}\right) \sum_{r=1}^{t} p^{r} \eta_{p}(r) \log (p) .
$$

By induction, we have, for the last summand,

$$
\begin{aligned}
& H_{0}\left(m_{0} ; D\right) \cdot\left(1-\chi_{d}(p) p^{-1}\right) \sum_{r=1}^{t} p^{r} \eta_{p}(r) \log (p) \\
& \quad=H_{0}\left(m_{0} ; D\right) \cdot\left(\sum_{r=1}^{t} p^{r}\left(1-\chi_{d}(p) p^{-1}\right)+1\right)\left(-t-\beta_{p}(t)\right) \log (p) \\
& \quad=H_{0}(m ; D) \cdot\left(-t-\beta_{p}(t)\right) \log (p) .
\end{aligned}
$$

Hence,

$$
\mathcal{L}(m)=\frac{p^{t+1}-\chi_{d}(p) p^{t}+\chi_{d}(p)-1}{p-1} \cdot \mathcal{L}\left(m_{0}\right)+H_{0}(m ; D) \cdot\left(-t-\beta_{p}(t)\right) \log (p) .
$$




\section{DeRivatives of Eisenstein SERIES AND FALtings Heights}

Now recall from (8.12) and Lemma 8.5 that

$$
H_{0}(m ; D)=\frac{h(d)}{w(d)} \prod_{q \nmid D} \frac{q^{t+1}-\chi_{d}(q) q^{t}+\chi_{d}(q)-1}{q-1} .
$$

It follows that

$$
\frac{H_{0}(m ; D)}{H_{0}\left(m_{0} ; D\right)}=\frac{p^{t+1}-\chi_{d}(p) p^{t}+\chi_{d}(p)-1}{p-1} .
$$

From the definition of $\mathcal{R}(m)$ we have

$$
\begin{aligned}
\mathcal{R}(m) & =\frac{H_{0}(m, D)}{H_{0}\left(m_{0} ; D\right)} \cdot \mathcal{R}\left(m_{0}\right)+H_{0}(m, D) \cdot\left(-t-\beta_{p}(t)\right) \log (p) \\
& =\frac{p^{t+1}-\chi_{d}(p) p^{t}+\chi_{d}(p)-1}{p-1} \cdot \mathcal{R}\left(m_{0}\right)+H_{0}(m ; D) \cdot\left(-t-\beta_{p}(t)\right) \log (p) .
\end{aligned}
$$

Comparing (10.78) with (10.81), the induction hypothesis $\mathcal{L}\left(m_{0}\right)=\mathcal{R}\left(m_{0}\right)$ implies the assertion.

The following result is well known (cf., for example, [Col93]).

Proposition 10.10. With the normalization given by (10.14) above, the Faltings height $h_{\mathrm{Fal}}(E)$ of an elliptic curve $E$ with $C M$ by $O_{k}$ is given by

$$
\begin{aligned}
2 h_{\mathrm{Fal}}(E) & =-\frac{1}{2} \log (d)-\frac{L^{\prime}\left(0, \chi_{d}\right)}{L\left(0, \chi_{d}\right)} \\
& =\frac{1}{2} \log (d)-\frac{w(d)}{2 h(d)} \sum_{a=1}^{d-1} \chi_{d}(a) \log \Gamma\left(\frac{a}{d}\right) \\
& =\frac{1}{2} \log (d)+\frac{L^{\prime}\left(1, \chi_{d}\right)}{L\left(1, \chi_{d}\right)}-\log (2 \pi)-\gamma .
\end{aligned}
$$

Remark 10.11. The value for $2 h_{\mathrm{Fal}}(E)$ in Colmez [Col93, p. 633], is our $2 h_{\mathrm{Fal}}(E)-\log (2 \pi)$ due to a difference in the normalization of the metric on the Hodge bundle.

Our renormalized Faltings height is then given by

$$
\begin{aligned}
2 h_{\mathrm{Fal}}^{*}(E) & =2 h_{\mathrm{Fal}}(E)+\frac{1}{2} \log (\pi)+\frac{1}{2} \gamma+\log (2) \\
& =\frac{1}{2} \log (d)+\frac{L^{\prime}\left(1, \chi_{d}\right)}{L\left(1, \chi_{d}\right)}-\frac{1}{2} \log (\pi)-\frac{1}{2} \gamma .
\end{aligned}
$$

Combining these facts, we have the following.

COROLlary 10.12. The contribution of the 'horizontal' part to the pairing $\langle\hat{\mathcal{Z}}(m, v), \hat{\omega}\rangle$ is

$$
\begin{aligned}
h_{\hat{\omega}}\left(\mathcal{Z}(m)^{\text {horiz }}\right)= & 2 \delta(d ; D) H_{0}(m ; D) \\
& \times\left[\frac{1}{2} \log (d)+\frac{L^{\prime}\left(1, \chi_{d}\right)}{L\left(1, \chi_{d}\right)}-\frac{1}{2} \log (\pi)-\frac{1}{2} \gamma+\sum_{\substack{p \\
p \nmid D}}\left(\log |n|_{p}-\frac{b_{p}^{\prime}(n, 0 ; D)}{b_{p}(n, 0 ; D)}\right)\right] .
\end{aligned}
$$

Proof of Theorem 7.2. Looking back at the end of $\S$ 8, we see that the expression of Corollary 10.12 for $h_{\hat{\omega}}\left(\mathcal{Z}(m)^{\text {horiz }}\right)$ coincides exactly with the sum of (8.40) and (8.42). The remaining terms will be considered in the next two sections. 


\section{S. S. Kudla, M. RApoport And T. YAnG}

\section{Contributions of vertical components}

In this section we fix a prime number $p$ with $p \mid D(B)$. We wish to determine the quantity $\operatorname{deg}\left(\omega \mid \mathcal{Z}(m)_{p}^{\text {vert }}\right)$ (cf. (9.14)), using the results of [KR00a].

We describe $\mathcal{Z}(m) \times_{\operatorname{Spec}} \mathbb{Z} \operatorname{Spec} W\left(\overline{\mathbb{F}}_{p}\right)$ in terms of the $p$-adic uniformization of

$$
\mathcal{M} \times \text { Spec } \mathbb{Z} \operatorname{Spec} W\left(\overline{\mathbb{F}}_{p}\right)
$$

(cf. $\S 2$ ). To this end we fix $x \in O_{B^{\prime}}$ with $\operatorname{tr}^{\circ}(x)=0$ and $x^{2}=-m$. As in $\S 2$ we identify $B^{\prime} \otimes \mathbb{A}_{f}^{p}$ with $B \otimes \mathbb{A}_{f}^{p}, H\left(\mathbb{A}_{f}^{p}\right)$ with $H^{\prime}\left(\mathbb{A}_{f}^{p}\right)$ and $K^{p}$ with $K^{\prime p}$. Put

$$
I(x)=\left\{g K^{p} \in H^{\prime}\left(\mathbb{A}_{f}^{p}\right) / K^{\prime, p} \mid g^{-1} x g \in \hat{O}_{B^{\prime}}^{p}\right\} .
$$

We also use the abbreviation $\hat{\Omega}_{W\left(\overline{\mathbb{F}}_{p}\right)}$ for $\hat{\Omega}^{2} \times_{\operatorname{Spf} \mathbb{Z}_{p}} \operatorname{Spf} W\left(\overline{\mathbb{F}}_{p}\right)$. Let $\tilde{x}=x$, if $\operatorname{ord}_{p}(m)=0(\operatorname{respectively}$ $\tilde{x}=1+x$, if $\left.\operatorname{ord}_{p}(m)>0\right)$. Let

$$
Z(x)=\left(\hat{\Omega}_{W\left(\overline{\mathbb{F}}_{p}\right)} \times \mathbb{Z}\right)^{\tilde{x}}
$$

be the fixed point set of $\tilde{x} \in H^{\prime}\left(\mathbb{Q}_{p}\right)$. Denoting by $H_{x}^{\prime}$ the stabilizer of $x$ in $H^{\prime}$, we have [KR00a]

$$
\mathcal{Z}(m) \times_{\operatorname{Spec} \mathbb{Z}} \operatorname{Spec} W\left(\overline{\mathbb{F}}_{p}\right)=\left[H_{x}^{\prime}(\mathbb{Q}) \backslash I(x) \times Z(x)\right]
$$

(quotient in the sense of stacks). Since $\operatorname{ord}_{p} \operatorname{det}(\tilde{x})=0$, we have

$$
Z(x)=\hat{\Omega}_{W\left(\overline{\mathbb{F}}_{p}\right)}^{\tilde{x}} \times \mathbb{Z} .
$$

Since the set

$$
H_{x}^{\prime}\left(\mathbb{A}_{f}^{p}\right) \backslash\left\{g \in H^{\prime}\left(\mathbb{A}_{f}^{p}\right) \mid g^{-1} x g \in \hat{O}_{B^{\prime}}^{p}\right\}
$$

is compact, the group $H_{x}^{\prime}\left(\mathbb{A}_{f}^{p}\right)$ has only finitely many orbits on $I(x)$. Let $g_{1}, \ldots, g_{r} \in H^{\prime}\left(\mathbb{A}_{f}^{p}\right)$ such that

$$
I(x)=\coprod_{i=1}^{r} H_{x}^{\prime}\left(\mathbb{A}_{f}^{p}\right) g_{i} K^{\prime, p}
$$

Then we may rewrite (11.3) as

$$
\coprod_{i=1}^{r}\left[H_{x}^{\prime}(\mathbb{Q}) \backslash\left(H_{x}^{\prime}\left(\mathbb{A}_{f}^{p}\right) /\left(K_{i}^{\prime, p} \cap H_{x}^{\prime}\left(\mathbb{A}_{f}^{p}\right)\right) \times \mathbb{Z} \times \hat{\Omega}_{W\left(\overline{\mathbb{F}}_{p}\right)}^{\tilde{x}}\right)\right],
$$

where $K_{i}^{\prime p}=g_{i} K^{\prime p} g_{i}^{-1}$.

Note that $H_{x}^{\prime}(\mathbb{Q}) \cong k^{\times}$, where $k=\mathbb{Q}(\sqrt{-m})$ is the imaginary quadratic field determined by $m$. Let us first consider the case where $p$ does not split in $k$. Then

$$
\operatorname{ord}_{p} \operatorname{det}\left(k_{p}^{\times}\right)=\delta_{p} \cdot \mathbb{Z},
$$

where $\delta_{p}=2$ if $p$ is unramified in $k$ and $\delta_{p}=1$ if $p$ is ramified in $k$. Let

$$
H_{x}^{\prime}(\mathbb{Q})^{1}=\left\{g \in H_{x}^{\prime}(\mathbb{Q}) \mid \operatorname{ord}_{p}(\operatorname{det}(g))=0\right\} .
$$

Then $H_{x}^{\prime}(\mathbb{Q})^{1}$ acts with finite stabilizer groups on $H_{x}^{\prime}\left(\mathbb{A}_{f}^{p}\right) /\left(K_{i}^{\prime, p} \cap H_{x}^{\prime}\left(\mathbb{A}_{f}^{p}\right)\right)$. Hence we may rewrite (11.3) as $\delta_{p}$ copies of

$$
\coprod_{i=1}^{r}\left[H_{x}^{\prime}(\mathbb{Q})^{1} \backslash\left(H^{\prime}\left(\mathbb{A}_{f}^{p}\right) /\left(K_{i}^{\prime, p} \cap H_{x}^{\prime}\left(\mathbb{A}_{f}^{p}\right)\right)\right)\right] \times \hat{\Omega}_{W\left(\overline{\mathbb{F}}_{p}\right)}^{\tilde{x}}
$$

(here the first factor is taken in the sense of stacks). 


\section{DeRivatives of Eisenstein SERIES AND FALtings Heights}

Appealing now to [KR00a, Proposition 3.2], we obtain the following expression for the vertical components of $\mathcal{Z}(m)$ :

$$
\begin{array}{r}
\mathcal{Z}(m)^{\text {vert }} \times_{\text {Spec } \mathbb{Z}} \operatorname{Spec} W\left(\overline{\mathbb{F}}_{p}\right)=\mathbb{Z} / \delta_{p} \mathbb{Z} \times \coprod_{i=1}^{r}\left[H_{x}^{\prime}(\mathbb{Q})^{1} \backslash\left(H^{\prime}\left(\mathbb{A}_{f}^{p}\right) /\left(K_{i}^{\prime, p} \cap H_{x}^{\prime}\left(\mathbb{A}_{f}^{p}\right)\right)\right)\right] \\
\times\left(\sum_{[\Lambda] \in \mathcal{B}} \operatorname{mult}_{[\Lambda]}(x) \cdot \mathbb{P}_{[\Lambda]}\right) .
\end{array}
$$

Here $[\Lambda]$ ranges over the vertices of the Bruhat-Tits tree of $\mathrm{PGL}_{2}\left(\mathbb{Q}_{p}\right)$ and the multiplicity with which the prime divisor $\mathbb{P}_{[\Lambda]}$ occurs is given by [KR00a, (3.9)] for $p \neq 2$ and by Proposition A.1 below for $p=2$.

Proposition 11.1. For any $[\Lambda] \in \mathcal{B}$ we have

$$
\operatorname{deg}\left(\omega \mid \mathbb{P}_{[\Lambda]}\right)=p-1 .
$$

Proof. Of course, here $\operatorname{deg}\left(\omega \mid \mathbb{P}_{[\Lambda]}\right)$ is shorthand for

$$
\operatorname{deg} i_{[\Lambda]}^{*}\left(\omega \otimes_{\mathbb{Z}} W\left(\overline{\mathbb{F}}_{p}\right)\right),
$$

where $i_{[\Lambda]}: \mathbb{P}_{[\Lambda]} \rightarrow \mathcal{M} \times_{\text {Spec } \mathbb{Z}} \operatorname{Spec} W\left(\overline{\mathbb{F}}_{p}\right)$ is the natural morphism. We write $O_{B_{p}}$ as

$$
O_{B_{p}}=\mathbb{Z}_{p^{2}}[\Pi] /\left(\Pi^{2}=p, \Pi a=a^{\sigma} \Pi, \forall a \in \mathbb{Z}_{p^{2}}\right) .
$$

For the inverse image of the universal abelian scheme $(\mathcal{A}, \iota)$ on $\mathcal{M} \times \operatorname{Spec} \mathbb{Z} \operatorname{Spec} W\left(\overline{\mathbb{F}}_{p}\right)$, we have

$$
\text { Lie } \mathcal{A}=\mathcal{L}_{0} \oplus \mathcal{L}_{1}
$$

where $\mathcal{L}_{i}=\left\{x \in \operatorname{Lie} \mathcal{A} \mid \iota(a) x=a^{\sigma^{-i}} x, \forall a \in \mathbb{Z}_{p^{2}}\right\}$.

Due to the determinant condition $(1.1)$, both $\mathcal{L}_{0}$ and $\mathcal{L}_{1}$ are line bundles on $\mathcal{M} \times_{\operatorname{Spec}} \mathbb{Z} \operatorname{Spec} W\left(\overline{\mathbb{F}}_{p}\right)$ and

$$
\omega \otimes_{\mathbb{Z}} W\left(\overline{\mathbb{F}}_{p}\right)=\mathcal{L}_{0}^{-1} \otimes \mathcal{L}_{1}^{-1}
$$

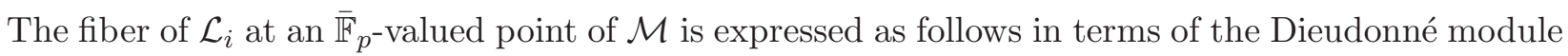
$M$ of the corresponding abelian variety,

$$
\mathcal{L}_{0}=M_{0} / V M_{1}, \quad \mathcal{L}_{1}=M_{1} / V M_{0} .
$$

Here $M=M_{0} \oplus M_{1}$ is the eigenspace decomposition under the action of $\mathbb{Z}_{p^{2}}$ analogous to (11.10).

To fix ideas assume that $\Lambda$ is even [KR00a]. Then for every $x \in \mathbb{P}_{[\Lambda]}\left(\overline{\mathbb{F}}_{p}\right)$ we have

$$
M_{0}=\Lambda \otimes_{\mathbb{Z}_{p}} W\left(\overline{\mathbb{F}}_{p}\right), \quad V M_{0}=\Pi M_{0}, \quad \mathcal{L}_{0 x}=M_{0} / \ell_{x},
$$

where $\ell_{x}$ is the line in $\Lambda \otimes_{\mathbb{Z}_{p}} \overline{\mathbb{F}}_{p}$ corresponding to $x$. It follows that

$$
i_{[\Lambda]}^{*}\left(\mathcal{L}_{0}\right)=\mathcal{O}_{\mathbb{P}_{[\Lambda]}}(1) .
$$

(It is $\mathcal{O}_{\mathbb{P}_{[\Lambda]}}(1)$ rather than $\mathcal{O}_{\mathbb{P}_{[\Lambda]}}(-1)$ since $\mathcal{L}_{0}$ obviously has global sections.) To calculate $i_{[\Lambda]}^{*}\left(\mathcal{L}_{1}\right)$, we use the exact sequence of coherent sheaves on $\hat{\Omega}_{W\left(\overline{\mathbb{F}}_{p}\right)}$

$$
0 \longrightarrow \mathcal{L}_{1} \stackrel{\Pi}{\longrightarrow} \mathcal{L}_{0} \longrightarrow \mathcal{O}_{\mathbb{P o d d}} \longrightarrow 0 .
$$

Here $\mathcal{O}_{\text {Podd }}$ denotes the structure sheaf of the closed subscheme of the special fiber [KR00a, $\left.\S 2\right]$,

$$
\bigcup_{[\Lambda] \text { odd }} \mathbb{P}_{[\Lambda]} \subset \hat{\Omega}_{W\left(\overline{\mathbb{F}}_{p}\right)} \otimes_{W\left(\overline{\mathbb{F}}_{p}\right)} \overline{\mathbb{F}}_{p} .
$$

This sequence remains exact after pulling back and yields

$$
0 \longrightarrow i_{[\Lambda]}^{*}\left(\mathcal{L}_{1}\right) \longrightarrow i_{[\Lambda]}^{*}\left(\mathcal{L}_{0}\right) \longrightarrow \mathcal{O}_{\mathbb{P}_{[\Lambda]}\left(\mathbb{F}_{p}\right)} \longrightarrow 0
$$




\section{S. S. Kudla, M. Rapoport And T. YAng}

(note that $i_{[\Lambda]}^{*}\left(\mathcal{L}_{1}\right)$ is torsion-free). Here we used the fact that

$$
\mathbb{P}_{[\Lambda]}\left(\mathbb{F}_{p}\right)=\mathbb{P}_{[\Lambda]} \cap\left(\bigcup_{[\Lambda] \text { odd }} \mathbb{P}_{[\Lambda]}\right)
$$

From (11.14) we obtain

$$
\begin{aligned}
\operatorname{deg} i_{[\Lambda]}^{*}\left(\mathcal{L}_{1}\right) & =\operatorname{deg} i_{[\Lambda]}^{*}\left(\mathcal{L}_{0}\right)-(p+1) \\
& =-p .
\end{aligned}
$$

Now the identity (11.11) yields the assertion. The case where $[\Lambda]$ is odd is similar.

Remark 11.2. Another proof of Proposition 11.1 may be obtained by using Proposition 3.2. Indeed,

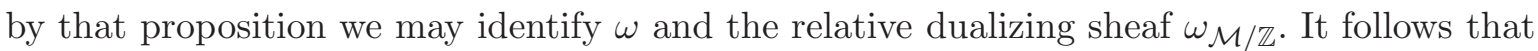

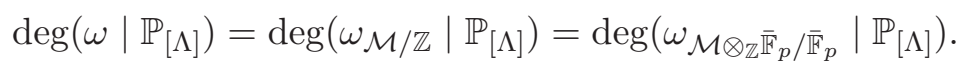

By expressing the dualizing sheaf $\omega_{\mathcal{M} \otimes_{\mathbb{Z}} \overline{\mathbb{F}}_{p} / \overline{\mathbb{F}}_{p}}$ explicitly, it is easy to calculate the last term.

Corollary 11.3. Let $k=\operatorname{ord}_{p} n$, where, as usual, $4 m=n^{2} d$. Then

$$
\sum_{[\Lambda] \in \mathcal{B}} \operatorname{mult}_{[\Lambda]}(x) \cdot \operatorname{deg}\left(\omega \mid \mathbb{P}_{[\Lambda]}\right)=\left\{\begin{array}{l}
-2 k+(p+1) \cdot \frac{p^{k}-1}{p-1} \quad \text { if } p \text { is unramified in } k \\
-2 k-2+2 \cdot \frac{p^{k+1}-1}{p-1} \quad \text { if } p \text { is ramified in } k .
\end{array}\right.
$$

Proof. It remains to calculate $\sum_{[\Lambda]} \operatorname{mult}_{[\Lambda]}(x)$. If $p$ is odd, this is an easy exercise using the results of $[$ KR00a, $\S 6]$. For instance, let $p$ be odd and unramified and put $\alpha=\operatorname{ord}_{p}(m)$ so that $\alpha=2 k$. Then

$$
\sum_{[\Lambda]} \operatorname{mult}_{[\Lambda]}(x)=\frac{\alpha}{2}+(p+1) \sum_{r=1}^{\frac{\alpha}{2}-1} p^{r-1}\left(\frac{\alpha}{2}-r\right)=\frac{-\alpha}{p-1}+\frac{p+1}{p-1} \cdot \frac{p^{\alpha / 2}-1}{p-1} .
$$

The case $p=2$ is handled in Appendix A.

It now remains to determine the degree of the discrete stack

$$
\coprod_{i=1}^{r}\left[H_{x}^{\prime}(\mathbb{Q})^{1} \backslash\left(H^{\prime}\left(\mathbb{A}_{f}^{p}\right) /\left(K_{i}^{\prime, p} \cap H_{x}^{\prime}\left(\mathbb{A}_{f}^{p}\right)\right)\right)\right] .
$$

Now the elements $g_{1}, \ldots, g_{r}$ are in one-to-one correspondence with the $\hat{O}_{B}^{p, \times}$-conjugacy classes of embeddings of the rings

$$
j^{p}: k \otimes \mathbb{A}_{f}^{p} \longrightarrow B \otimes \mathbb{A}_{f}^{p},
$$

with $x \in\left(j^{p}\right)^{-1}\left(\hat{\mathcal{O}}_{B}^{p}\right)$. To each embedding $j^{p}$ there is associated an order of $k$,

$$
O\left(j^{p}\right)=\left(\left(j^{p}\right)^{-1}\left(\hat{O}_{B}^{p}\right) \cdot O_{k_{p}}\right) \cap k .
$$

The conductor $c=c\left(j^{p}\right)$ of this order satisfies $(c, D(B))=1$ and $c \mid n$, because $x \in\left(j^{p}\right)^{-1}\left(O_{B}^{p}\right)$. Conversely, given $c$ with those two properties, there are precisely

$$
\prod_{\substack{\ell \mid D(B) \\ \ell \neq p}}\left(1-\chi_{d}(\ell)\right)
$$




\section{DeRivatives of Eisenstein SERIES AND FALtings Heights}

classes of embeddings $j^{p}$ yielding the order of conductor $c$. Finally, if $g_{i}$ yields the order of conductor $c$, the stack $\left[H_{x}^{\prime}(\mathbb{Q})^{1} \backslash\left(H^{\prime}\left(\mathbb{A}_{f}^{p}\right) / K_{i}^{\prime} \cap H_{x}^{\prime}\left(\mathbb{A}_{f}^{p}\right)\right)\right]$ may be identified with

$$
\left[k^{\times, 1} \backslash\left(\left(k \otimes \mathbb{A}_{f}^{p}\right)^{\times} / \hat{O}_{c^{2} d}^{p, \times}\right)\right]
$$

which has degree $h\left(c^{2} d\right) / w\left(c^{2} d\right)$. Summarizing these arguments, we therefore obtain

LEMMA 11.4.

$$
\begin{aligned}
\delta_{p} \cdot \operatorname{deg}\left(\coprod_{i=1}\left[H_{x}^{\prime}(\mathbb{Q})^{1} \backslash\left(H^{\prime}\left(\mathbb{A}_{f}^{p}\right) / K_{i}^{\prime, p} \cap H_{x}^{\prime}\left(\mathbb{A}_{f}^{p}\right)\right)\right]\right) & =\prod_{\ell \mid D(B)}\left(1-\chi_{d}(\ell)\right) \cdot \sum_{\substack{c \mid n \\
(c, D(B))=1}} h\left(c^{2} d\right) / w\left(c^{2} d\right) \\
& =\delta(d ; D(B)) \cdot H_{0}(m ; D(B)) .
\end{aligned}
$$

Now let us consider the case when $p$ splits in $k$. In this case $\operatorname{ord}_{p}\left(\operatorname{det}\left(k_{p}^{\times}\right)\right)=\mathbb{Z}$, but $H_{x}^{\prime}(\mathbb{Q})^{1}$ does not act with finite stabilizer groups on $H_{x}^{\prime}\left(\mathbb{A}_{f}^{p}\right) /\left(K_{i}^{\prime p} \cap H_{x}^{\prime}\left(\mathbb{A}_{f}^{p}\right)\right)$. Let $\epsilon(x) \in H_{x}^{\prime}(\mathbb{Q})=k^{\times}$ be an element whose localization in $k_{p}=\mathbb{Q}_{p} \oplus \mathbb{Q}_{p}$ has the valuation $(1,-1)$. Let $H_{x}^{\prime}(\mathbb{Q})^{1,1}$ be the subgroup of elements of $H_{x}^{\prime}(\mathbb{Q})$ which are units at $p$. Then $H_{x}^{\prime}(\mathbb{Q})^{1}=H_{x}^{\prime}(\mathbb{Q})^{1,1} \times\left\langle\epsilon(x)^{\mathbb{Z}}\right\rangle$ and $H_{x}^{\prime}(\mathbb{Q})^{1,1}$ acts with finite stabilizer groups on $H_{x}^{\prime}\left(A_{f}^{p}\right) /\left(K_{i}^{\prime p} \cap H_{x}^{\prime}\left(\mathbb{A}_{f}^{p}\right)\right)$, whereas $\epsilon(x)$ acts freely on $\hat{\Omega}_{W\left(\overline{\mathbb{F}}_{p}\right)}^{\tilde{x}}$ by translations by 2 on the 'apartment of central components' [KR00a]. We therefore obtain the following expression for (11.3) in this case:

$$
\left(\coprod_{i=1}^{r}\left[H_{x}^{\prime}(\mathbb{Q})^{1,1} \backslash\left(H_{x}^{\prime}\left(\mathbb{A}_{f}^{p}\right) /\left(K_{i}^{\prime, p} \cap H_{x}^{\prime}\left(\mathbb{A}_{f}^{p}\right)\right)\right)\right]\right) \times\left(\left\langle\epsilon(x)^{\mathbb{Z}}\right\rangle \backslash \hat{\Omega}_{W\left(\overline{\mathbb{F}}_{p}\right)}^{\tilde{x}}\right) .
$$

The same analysis as before yields

$$
\begin{aligned}
\operatorname{deg} & \left(\coprod_{i=1}^{r}\left[H_{x}^{\prime}(\mathbb{Q})^{1,1} \backslash\left(H_{x}^{\prime}\left(\mathbb{A}_{f}^{p}\right) /\left(K_{i}^{\prime, p} \cap H_{x}^{\prime}\left(\mathbb{A}_{f}^{p}\right)\right)\right)\right]\right) \\
& =\prod_{\substack{\ell \mid D(B) \\
\ell \neq p}}\left(1-\chi_{d}(\ell)\right) \cdot \sum_{\substack{c \mid n \\
(c, D(B))=1}} h\left(c^{2} d\right) / w\left(c^{2} d\right) \\
& =\delta(d ; D(B) / p) \cdot H_{0}(m ; D(B)) .
\end{aligned}
$$

Using Proposition 11.1 we have

$$
\operatorname{deg}\left(\omega \mid\left(\left\langle\epsilon(x)^{\mathbb{Z}}\right\rangle \backslash \hat{\Omega}_{W\left(\overline{\mathbb{F}}_{p}\right)}^{\tilde{x}}\right)\right)=2(p-1) \sum_{[\Lambda]} \operatorname{mult}_{[\Lambda]}(x) .
$$

The right-hand side is explained as follows. The action of $\epsilon(x)$ on the apartment corresponding to $k_{p}^{\times}$ has a fundamental domain consisting of two vertices. Fix a vertex $\left[\Lambda_{0}\right]$ in the apartment. Then the sum in (11.21) ranges over all vertices $[\Lambda]$ for which $\left[\Lambda_{0}\right]$ is the closest vertex in the apartment. This sum can again be evaluated using [KR00a, 3.9] for $p$ odd (respectively Appendix A for $p=2$ ).

We summarize our findings in the following theorem.

Theorem 11.5. Let $k=\operatorname{ord}_{p}(n)$, where, as usual, $4 m=n^{2} d$.

i) If $p$ splits in $k$, then

$$
\operatorname{deg}\left(\omega \mid \mathcal{Z}(m)_{p}^{\text {vert }}\right)=2 H_{0}(m ; D(B)) \delta(d ; D(B) / p) \cdot\left(p^{k}-1\right) .
$$


ii)

$$
\operatorname{deg}\left(\omega \mid \mathcal{Z}(m)_{p}^{\mathrm{vert}}\right)=2 H_{0}(m ; D(B)) \delta(d ; D(B)) \cdot \begin{cases}-k+\frac{(p+1)\left(p^{k}-1\right)}{2(p-1)} & \text { if } \chi_{d}(p)=-1 \\ -1-k+\frac{p^{k+1}-1}{p-1} & \text { if } \chi_{d}(p)=0\end{cases}
$$

Proof of Theorem 7.2 (continued). In the case $\chi_{d}(p)=1$, the quantity

$$
\operatorname{deg}\left(\omega \mid \mathcal{Z}(m)_{p}^{\operatorname{vert}}\right) \log (p) \cdot q^{m}
$$

coincides exactly with part ii of Theorem 8.8. On the other hand, in the cases $\chi_{d}(p)=-1$ and $\chi_{d}(p)=0$, we find that

$$
\operatorname{deg}\left(\omega \mid \mathcal{Z}(m)_{p}^{\operatorname{vert}}\right) \log (p) \cdot q^{m}=2 \delta(d ; D) H_{0}(m ; D) K_{p} \log (p) \cdot q^{m},
$$

where $K_{p}$ is as in Theorem 8.8. Thus, summing on $p \mid D$, we obtain (8.42).

\section{Appendix A. The case $p=2$}

In [KR00a], we made the blanket assumption $p \neq 2$. In this appendix we indicate the modifications needed to arrive at the formulas given in Theorem 11.5 in the case $p=2$.

We use the same notation as in [KR00a]. We fix a special endomorphism $j \in V$ with $q(j)=j^{2} \in$ $\mathbb{Z}_{2} \backslash\{0\}$. We denote by $Z(j)$ the associated closed formal subscheme of the Drinfeld moduli space $\mathcal{M} \simeq \hat{\Omega} \times{ }_{\operatorname{Spf} \mathbb{Z}_{2}} \operatorname{Spf} W\left(\overline{\mathbb{F}}_{2}\right)$. We content ourselves with giving the structure of the divisor $Z(j)^{\text {pure }}$ associated to $Z(j)$ [KR00a, $\S 4$ ]. Our discussion proceeds by distinguishing cases. Let $k=\mathbb{Q}_{2}(j)$ (hence in the global case $k$ is the localization at 2 of the imaginary quadratic field). Let $\mathcal{O}=\mathcal{O}_{k}$ be the ring of integers in $k$. We write as usual

$$
q(j)=\varepsilon \cdot 2^{\alpha}, \quad \varepsilon \in \mathbb{Z}_{2}^{\times}, \quad \alpha \geqslant 0 .
$$

We define $k \geqslant 0$ by

$$
\alpha+2=2 k+\operatorname{ord}_{2}(d)
$$

where $d$ denotes the discriminant of $\mathcal{O} / \mathbb{Z}_{2}$. Note that in the global context, when $\mathcal{Z}(m)$ is $p$-adically uniformized by $Z(j)$ (cf. (11.3) above), then $\alpha=\operatorname{ord}_{p}(m)$. If we write as usual $4 m=n^{2} d$, then $k=\operatorname{ord}_{p}(n)$.

We then have the cases shown in Table A1.

TABLE A1.

\begin{tabular}{ccccc}
\hline Case & $q(j)$ & 2 in $k$ & Value of $k$ & $\mathcal{B}^{\mathcal{O}^{\times}}$ \\
\hline 1 & $2 \mid \alpha, \varepsilon \equiv 1(8)$ & split & $\frac{\alpha}{2}+1$ & $\mathcal{A}$ \\
2 & $2 \mid \alpha, \varepsilon \equiv 5(8)$ & unramified & $\frac{\alpha}{2}+1$ & $\left\{\left[\Lambda_{0}\right]\right\}$ \\
3 & $2 \mid \alpha, \varepsilon \equiv-1(4)$ & ramified & $\frac{\alpha}{2}$ & $\left\{\left[\Lambda_{0}\right],\left[\Lambda_{1}\right]\right\}$ \\
4 & $2 \nmid \alpha$ & ramified & $\frac{\alpha-1}{2}$ & $\left\{\left[\Lambda_{0}\right],\left[\Lambda_{1}\right]\right\}$ \\
\hline
\end{tabular}

We explain the last column in this table. In cases 1 and 2 , writing $j=2^{\alpha / 2} \cdot \bar{j}$, the index of $\mathbb{Z}_{2}[\bar{j}]$ in $\mathcal{O}$ and the index of $\mathbb{Z}_{2}[\bar{j}]^{\times}$in $\mathcal{O}^{\times}$is 2 . In case $1, \mathcal{O}$ is defined as $\mathbb{Z}_{2} \oplus \mathbb{Z}_{2} \cdot(1+\bar{j}) / 2$. In this case, 


\section{DeRIVATIVES OF EISENSTEIN SERIES AND FAltings Heights}

the fixed point set of $\mathcal{O}^{\times}$is the apartment $\mathcal{A}$ in $\mathcal{B}$ corresponding to the split Cartan subgroup $k^{\times}$ of $\mathrm{GL}_{2}\left(\mathbb{Q}_{2}\right)$, and the fixed point set of $j$ is

$$
\mathcal{B}^{j}=\{[\Lambda] \mid d([\Lambda], \mathcal{A}) \leqslant 1\} .
$$

In case 2 , the fixed point set of $\mathcal{O}^{\times}$is the vertex corresponding to the lattice $\Lambda_{0}=\mathcal{O}$ in $\mathbb{Q}_{2}^{2}$. Let $\tilde{j}=(1+\bar{j}) / 2$ and denote by $\left[\Lambda_{1}\right]$ the vertex corresponding to the lattice $\Lambda_{1}=\mathbb{Z}_{2}[\bar{j}]$. Then

$$
\mathcal{B}^{j}=\left\{\left[\Lambda_{0}\right],\left[\Lambda_{1}\right],\left[\tilde{j}\left(\Lambda_{1}\right)\right],\left[\tilde{j}^{2}\left(\Lambda_{1}\right)\right]\right\}=\left\{[\Lambda] \mid d\left([\Lambda],\left[\Lambda_{0}\right]\right) \leqslant 1\right\} .
$$

In cases 3 and 4 we write $j=2^{[\alpha / 2]} \bar{j}$. Then we have $\mathcal{O}=\mathbb{Z}_{2}[\bar{j}]$. The fixed point set of $\mathcal{O}^{\times}$consists of the vertices corresponding to the lattices $\Lambda_{0}=\mathcal{O}$ and $\Lambda_{1}=\pi \mathcal{O}$, where $\pi$ denotes a uniformizer in $\mathcal{O}$. In case 3 this coincides with the fixed point set of $j$, whereas, in case $4, j$ permutes the two vertices $\left[\Lambda_{0}\right]$ and $\left[\Lambda_{1}\right]$ so that $\mathcal{B}^{j}$ consists of the midpoint of the edge formed by $\left[\Lambda_{0}\right]$ and $\left[\Lambda_{1}\right]$.

To formulate the theorem we write the divisor as usual as a sum of a vertical part and a horizontal part,

$$
Z(j)^{\text {pure }}=Z(j)^{\text {vert }}+Z(j)^{\text {horiz }} .
$$

Proposition A.1.

i) Let

$$
Z(j)^{\text {vert }}=\sum_{[\Lambda] \in \mathcal{B}} \operatorname{mult}_{[\Lambda]}(j) \cdot \mathbb{P}_{[\Lambda]} .
$$

Then the multiplicity $\operatorname{mult}_{[\Lambda]}(j)$ is given by

$$
\operatorname{mult}_{[\Lambda]}(j)=\max \left(k-d\left([\Lambda], \mathcal{B}^{\mathcal{O}^{\times}}\right), 0\right) .
$$

ii) In case $1, Z(j)^{\text {horiz }}=0$. In case $2, Z(j)^{\text {horiz }}$ is isomorphic to the disjoint union of two copies of Spf $W\left(\overline{\mathbb{F}}_{2}\right)$ and meets the special fiber in two ordinary special points of $\mathbb{P}_{\left[\Lambda_{0}\right]}$. In cases 3 and 4 , $Z(j)^{\text {horiz }}$ is isomorphic to $\mathrm{Spf} W^{\prime}$, where $W^{\prime}$ is the ring of integers in a ramified quadratic extension of $W\left(\overline{\mathbb{F}}_{2}\right)$, and meets the special fiber in the superspecial point corresponding to the midpoint of the edge formed by $\left[\Lambda_{0}\right]$ and $\left[\Lambda_{1}\right]$.

Proof. We first determine $Z(j) \cap\left(\hat{\Omega}_{[\Lambda]} \times_{\operatorname{Spf} \mathbb{Z}_{2}} \operatorname{Spf} W\left(\overline{\mathbb{F}}_{2}\right)\right)$ for a vertex $[\Lambda]$ where the intersection is non-empty. Let $m=\max \left\{r ; j(\Lambda) \subset 2^{r} \Lambda\right\}$. Then

$$
m=\frac{\alpha}{2}-d\left([\Lambda], \mathcal{B}^{j}\right)
$$

(cf. [KR00a, Lemma 2.8]). After choosing a basis of $\Lambda$ we may write

$$
j=2^{m} \cdot\left(\begin{array}{cc}
\bar{a} & \bar{b} \\
\bar{c} & -\bar{a}
\end{array}\right)=2^{m} \cdot \bar{j}
$$

where $\bar{a}, \bar{b}, \bar{c}$ are not simultaneously divisible by $p$. The equation of $Z(j)$ on $\hat{\Omega}_{[\Lambda]} \times_{\operatorname{Spf}} \mathbb{Z}_{2} \operatorname{Spf} W\left(\overline{\mathbb{F}}_{2}\right)=$ $\operatorname{Spf} W\left(\overline{\mathbb{F}}_{2}\right)\left[T,\left(T^{2}-T\right)^{-1}\right]^{\wedge}$ is given by

$$
2^{m} \cdot\left(\bar{b} T^{2}-2 \bar{a} T-\bar{c}\right)=0
$$

(cf. [KR00a, (3.5)]). We now distinguish two cases.

Case $A: 2 \mid \bar{b}$ and $2 \mid \bar{c}$. Then $2 \nmid \bar{a}$ and we may write (A.7) in the form

$$
2^{m+1} \cdot\left(\bar{b}_{0} T^{2}-\bar{a} T-\bar{c}_{0}\right)=0,
$$

where $\bar{b}=2 \bar{b}_{0}$ and $\bar{c}=2 \bar{c}_{0}$.

Hence, in this case the multiplicity $\operatorname{mult}_{[\Lambda]}(j)$ equals $m+1$. However, in this case $\bar{j} \in \mathrm{GL}_{2}\left(\mathbb{Z}_{2}\right)$ and hence $[\Lambda]$ is fixed by $j$. We check now case by case when case A can occur. Case 4 can be 


\section{S. S. Kudla, M. Rapoport And T. YAng}

excluded right away since in this case no vertex is fixed by $j$. In case 3 , let $[\Lambda]=\left[\Lambda_{0}\right]$ with the notation introduced in this case, i.e. $\Lambda_{0}=\mathcal{O}=\mathbb{Z}_{2}[\bar{j}]$. Choosing as basis $1, \bar{j}$ we see that $j$ is given by the matrix

$$
j=2^{\alpha / 2} \cdot\left(\begin{array}{ll}
0 & \varepsilon \\
1 & 0
\end{array}\right)=2^{m} \bar{j}
$$

hence case A does not occur for $\left[\Lambda_{0}\right]$. The case when $[\Lambda]=\left[\Lambda_{1}\right]$ with $\Lambda_{1}=\pi \mathcal{O}$ is identical and hence case $\mathrm{A}$ does not occur in case 3 .

In case 2 , the vertex $\left[\Lambda_{1}\right]$ with $\Lambda_{1}=\mathbb{Z}_{2}[\bar{j}]$ is excluded for the same reason, and hence so are $\left[\tilde{j}\left(\Lambda_{1}\right)\right]$ and $\left[\tilde{j}^{2}\left(\Lambda_{1}\right)\right]$. Now let us consider the vertex $\left[\Lambda_{0}\right]$, with $\Lambda_{0}=\mathcal{O}$. We may choose the basis $1,(1+\bar{j}) / 2$ of $\Lambda_{0}$ and then $j$ is given by the matrix

$$
j=2^{\alpha / 2} \cdot\left(\begin{array}{cc}
-1 & 2 \lambda \\
2 & 1
\end{array}\right), \quad \text { where } \varepsilon-1=4 \lambda .
$$

Hence case A applies here. Furthermore, in this case, the second factor in (A.8) is equal to

$$
\lambda T^{2}+T-1 .
$$

Since, in case 2 , we have $\lambda \in \mathbb{Z}_{2}^{\times}$, the ring $\mathbb{Z}_{2}[T] /\left(\lambda T^{2}+T-1\right)$ is the ring of integers in an unramified quadratic extension of $\mathbb{Q}_{2}$ and the zeros of the polynomial $T^{2}+T-1 \in \mathbb{F}_{2}[T]$ lie in $\mathbb{F}_{4} \backslash \mathbb{F}_{2}$ and define two ordinary special points of $\mathbb{P}_{\left[\Lambda_{0}\right]}$.

In case 1 the analysis is similar to case 2 . First one checks that if $[\Lambda] \notin \mathcal{B}^{\mathcal{O}^{\times}}$, then case A does not occur. If $[\Lambda] \in \mathcal{B}^{\mathcal{O}^{\times}}$, then after replacing $[\Lambda]$ by $[g \Lambda]$ for some $g \in k^{\times}$we may assume that either $[\Lambda]=\left[\Lambda_{0}\right]$ with $\Lambda_{0}=\mathcal{O}=\langle 1,(1+j) / 2\rangle$ or $[\Lambda]=\left[\Lambda_{0}^{\prime}\right]$ with $\Lambda_{0}^{\prime}=\langle 2,(1+\bar{j}) / 2\rangle$. In the first case, the matrix of $j$ is given by (A.10) and hence we are in case A. The second factor in (A.8) is equal to

$$
\lambda T^{2}+T-1 \equiv T-1 \bmod 2,
$$

since $2 \mid \lambda$ in case 1 . It follows that $Z(j)^{\text {horiz }} \cap\left(\hat{\Omega}_{\left[\Lambda_{0}\right]} \times_{\operatorname{Spf} \mathbb{Z}_{2}} \operatorname{Spf} W\left(\overline{\mathbb{F}}_{2}\right)\right)=\emptyset$. In the second case the matrix of $j$ is given by

$$
j=2^{\alpha / 2} \cdot\left(\begin{array}{cc}
-1 & \lambda \\
4 & 1
\end{array}\right) .
$$

Again we are in case A, since $2 \mid \lambda$. The second factor in (A.8) is equal to

$$
\lambda_{0} T^{2}+T-2 \equiv \lambda_{0} T^{2}+T \bmod 2,
$$

where we have set $\lambda=2 \lambda_{0}$. Since $T^{2}-T$ is invertible on $\hat{\Omega}_{\left[\Lambda_{0}^{\prime}\right]}$, we again have $\left.Z(j)\right)^{\text {horiz }} \cap\left(\hat{\Omega}_{\left[\Lambda_{0}^{\prime}\right]} \times{ }_{\operatorname{Spf}} \mathbb{Z}_{2}\right.$ $\left.\operatorname{Spf} W\left(\overline{\mathbb{F}}_{2}\right)\right)=\emptyset$. This concludes our analysis of case A.

Case $B: 2 \nmid \bar{b}$ or $2 \nmid \bar{c}$. In this case, the second factor of (A.7) is not divisible by 2, and hence $\operatorname{mult}_{[\Lambda]}(j)=m$. At this point we have shown that for any $[\Lambda] \in \mathcal{B}$ the multiplicity $\operatorname{mult}_{[\Lambda]}(j)$ is given by the formula in part i. Indeed, this follows by listing the fixed point sets of $j$ case by case, and the expressions for $k$ and $m$ case by case (cf. (A.5)) and comparing them with the multiplicities calculated above.

Now let us analyze the second factor in (A.7) in case B. Its image in $\mathbb{F}_{2}[T]$ is

$$
\bar{b} T^{2}-\bar{c},
$$

hence is equal to either $T^{2}-1,1$, or $T^{2}$. In all cases $Z(j)^{\text {horiz }} \cap\left(\hat{\Omega}_{[\Lambda]} \times_{\operatorname{Spf} \mathbb{Z}_{2}} \operatorname{Spf} W\left(\overline{\mathbb{F}}_{2}\right)\right)=\emptyset$.

Now we determine $Z(j)$ horiz $\cap\left(\hat{\Omega}_{\Delta} \times_{\operatorname{Spf} \mathbb{Z}_{2}} \operatorname{Spf} W\left(\overline{\mathbb{F}}_{2}\right)\right)$ for an edge $\Delta=\left\{[\Lambda],\left[\Lambda^{\prime}\right]\right\}$, where the intersection is non-empty. As in the proof of Proposition 3.3 in [KR00a] we see that this intersection is non-empty only when $d\left([\Lambda], \mathcal{B}^{j}\right)=d\left(\left[\Lambda^{\prime}\right], \mathcal{B}^{j}\right)$. In cases 3 and 4 , we therefore must have

$$
[\Lambda]=\left[\Lambda_{0}\right], \quad\left[\Lambda^{\prime}\right]=\left[\Lambda_{1}\right]
$$




\section{DeRivatives of Eisenstein SERIES AND FALtings Heights}

In case 3 , noting that $1+\bar{j}$ is a uniformizer of $\mathcal{O}$, we take as basis in standard form for $\Lambda_{0}, \Lambda_{1}$

$$
\Lambda_{0}=\langle 1,1+\bar{j}\rangle, \quad \Lambda_{1}=\langle 2,1+\bar{j}\rangle .
$$

In terms of the basis $1,1+\bar{j}$ of $\Lambda_{0}$, the matrix of $j$ is

$$
j=p^{\alpha / 2} \cdot\left(\begin{array}{cc}
-1 & -2 \cdot(1-2 \lambda) \\
1 & 1
\end{array}\right), \quad \text { where } \varepsilon=4 \lambda-1 .
$$

By [KR00a] the equations for $Z(j) \cap\left(\hat{\Omega}_{\Delta} \times_{\operatorname{Spf} \mathbb{Z}_{2}} \operatorname{Spf} W\left(\overline{\mathbb{F}}_{2}\right)\right)$ in

$$
\hat{\Omega}_{\Delta} \times_{\operatorname{Spf} \mathbb{Z}_{2}} \operatorname{Spf} W\left(\overline{\mathbb{F}}_{2}\right)=\operatorname{Spf} W\left(\overline{\mathbb{F}}_{2}\right)\left[T_{0}, T_{1},\left(1-T_{0}\right)^{-1},\left(1-T_{1}\right)^{-1}\right]^{\wedge} /\left(T_{0} T_{1}-2\right)
$$

are given by

$$
\begin{aligned}
& p^{\alpha / 2} \cdot T_{0}\left(-(1-2 \lambda) T_{0}+2-T_{1}\right)=0, \\
& p^{\alpha / 2} \cdot T_{1}\left(-(1-2 \lambda) T_{0}+2-T_{1}\right)=0 .
\end{aligned}
$$

Hence, $Z(j)^{\text {horiz }}$ is defined by the second factor in these equations. Now putting

$$
\mu=(-(1-2 \lambda))^{-1} \in \mathbb{Z}_{2}^{\times},
$$

we obtain

$$
Z(j)^{\text {horiz }}=\operatorname{Spf} W\left(\overline{\mathbb{F}}_{2}\right)\left[T_{0}\right] /\left(T_{0}^{2}+2 \mu T_{0}-2 \mu\right) .
$$

Since $T_{0}^{2}+2 \mu T_{0}-2 \mu$ is an Eisenstein polynomial, we see that $Z(j)^{\text {horiz }}$ is the formal spectrum of the ring of integers in a ramified quadratic extension of $W\left(\overline{\mathbb{F}}_{2}\right)$ and it meets the special fiber

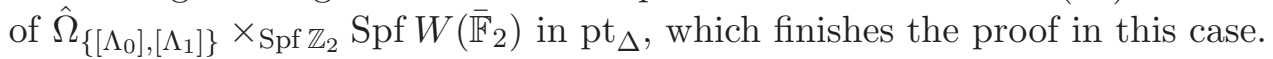

Case 4 is similar to the case of [KR00a, p. 180]..$^{5}$ In this case we have

$$
j\left(\Lambda_{0}\right)=2^{(\alpha-1) / 2} \cdot \Lambda_{1}, \quad j\left(\Lambda_{1}\right)=2^{(\alpha+1) / 2} \cdot \Lambda_{0} .
$$

Hence, as in [KR00a], we can write $j$ in terms of standard coordinates for $\Lambda_{0}, \Lambda_{1}$

$$
j=2^{(\alpha-1) / 2} \cdot\left(\begin{array}{cc}
\bar{a} & \bar{b} \\
\bar{c} & -\bar{a}
\end{array}\right), \quad \text { with } \bar{b}=2 \cdot \bar{b}_{0}
$$

and where $2 \mid \bar{a}$ and $\bar{b}_{0}$ and $\bar{c}$ are units. Hence, $Z(j)^{\text {horiz }}$ is isomorphic to

$$
\text { Spf } W\left(\overline{\mathbb{F}}_{2}\right)\left[T_{0}, T_{1},\left(1-T_{0}\right)^{-1},\left(1-T_{1}\right)^{-1}\right]^{\widehat{ }} /\left(T_{0} T_{1}-2, \bar{b}_{0} T_{0}-2 \bar{a}-\bar{c} T_{1}\right) .
$$

Putting $\mu=\left(b_{0} / \bar{c}\right)^{-1} \in \mathbb{Z}_{2}^{\times}$and $\nu=\bar{a} / \bar{c}$, we see that

$$
Z(j)^{\text {horiz }}=\operatorname{Spf} W\left(\overline{\mathbb{F}}_{2}\right)\left[T_{0}\right] /\left(T_{0}^{2}-2 \mu \nu T_{0}-2 \mu\right)
$$

which yields the assertion as in case 3 .

In case 2 , we must have $\Delta=\left\{\left[\Lambda_{0}\right],\left[\tilde{j}^{i}\left(\Lambda_{1}\right)\right]\right\}$, where $\Lambda_{0}=\mathcal{O}$ and $\Lambda_{1}=\mathbb{Z}_{2}[\bar{j}]$ and for some $i=0,1,2$. Let us consider the case $i=0$, the other cases being similar. In this case we take as standard bases

$$
\Lambda_{0}=\left\langle\frac{1+\bar{j}}{2}, 1\right\rangle, \quad \Lambda_{1}=\left\langle 2 \cdot \frac{1+\bar{j}}{2}, 1\right\rangle .
$$

${ }^{5}$ We note that at this point in [KR00a] there is a slight error. Equations (3.23) of [KR00a] do not define the same closed subscheme as Equations (3.22). The correct expression for $Z(j)^{h}$, replacing (3.24), is

$$
\begin{aligned}
& Z(j)^{h}=\operatorname{Spf} W\left[T_{0}, T_{1}\right]^{\widehat{N}} /\left(\bar{b}_{0} T_{0}-2 \bar{a}-\bar{c} T_{1}, T_{0} T_{1}-p\right) \\
& =\operatorname{Spf} W\left[T_{0}\right] /\left(T_{0}^{2}+\alpha T_{0}+\beta\right),
\end{aligned}
$$

where $\alpha \in p \mathbb{Z}_{p}$ and $\beta \in p \mathbb{Z}_{p}^{\times}$. The conclusions drawn from these corrected equations [KR00a, pp. 181-183] are unchanged. 


\section{S. S. Kudla, M. Rapoport And T. YAng}

But then, by (A.10), the equation for $Z(j)^{\text {horiz }}$ is given by

$$
T_{0}-1-\lambda T_{1}=0, \quad \text { where } \varepsilon-1=4 \lambda .
$$

It follows that $\mathrm{pt}_{\Delta} \notin Z(j)^{\text {horiz }}$, which finishes the proof in this case.

Finally, there is case 1 . In this case either $\Delta \subset \mathcal{A}$ or, after replacing $\Delta$ by $g \Delta$ where $g \in k^{\times}$, we may assume that $\Delta=\left\{\left[\Lambda_{0}\right],\left[\Lambda_{1}\right]\right\}$ where $\Lambda_{0}$ and $\Lambda_{1}$ are as in case 3 . The second alternative is treated as in case 3 above. If $\Delta \subset \mathcal{A}$, we may assume that

$$
\Lambda=\left\langle 1, \frac{1+\bar{j}}{2}\right\rangle, \quad \Lambda^{\prime}=\left\langle 2, \frac{1+\bar{j}}{2}\right\rangle .
$$

In this case $j$ is given by the matrix (A.10) which yields the following equations for $Z(j)$,

$$
\begin{aligned}
& p^{\alpha / 2} \cdot T_{0}\left(\lambda T_{0}+2-2 T_{1}\right)=0, \\
& p^{\alpha / 2} \cdot T_{1}\left(\lambda T_{0}+2-2 T_{1}\right)=0 .
\end{aligned}
$$

Since we are in case 1 , we may write $\lambda=2 \lambda_{0}$ in the defining equation $\varepsilon-1=4 \lambda$. It follows that, after pulling a 2 out of the last factor in both equations, $Z(j)^{\text {horiz }}$ is defined by the equation

$$
\lambda_{0} T_{0}+1-T_{1}=0,
$$

and again $\mathrm{pt}_{\Delta} \notin Z(j)^{\text {horiz }}$.

\section{Archimedean contributions}

In this section, we compute the additional contribution

$$
\kappa(m, v)=\frac{1}{2} \int_{[\Gamma \backslash D]} \Xi(m, v) c_{1}(\hat{\omega})
$$

to the height pairing coming from the fact that we are using non-standard Green functions defined in $[\operatorname{Kud} 97 \mathrm{~b}]$ for the cycles $\mathcal{Z}(m)$. Recall that, by (5.8) for $z \in D$, we have

$$
\Xi(m, v)(z)=\sum_{x \in L(m)} \xi\left(v^{\frac{1}{2}} x, z\right)
$$

where

$$
\xi(x, z)=-\operatorname{Ei}(-2 \pi R(x, z))
$$

Note that the quantity

$$
R(x, z)=-\left(\operatorname{pr}_{z}(x), \operatorname{pr}_{z}(x)\right),
$$

and hence $\xi(x, z)$, is independent of the orientation of the plane $z$. Also recall that $c_{1}(\hat{\omega})=\mu$.

Proposition 12.1.

i) If $m>0$, then

$$
\kappa(m, v)=2 \delta(d, D) H_{0}(m ; D) \cdot \frac{1}{2} J(4 \pi m v),
$$

where

$$
J(t)=\int_{0}^{\infty} e^{-t w}\left[(w+1)^{\frac{1}{2}}-1\right] w^{-1} d w
$$

is as in Theorem 8.8, and $H_{0}(m ; D)$ is given by (8.17).

ii) If $m<0$, then

$$
\kappa(m, v)=2 \delta(d ; D) H_{0}(m ; D) \frac{1}{4 \pi}|m|^{-\frac{1}{2}} v^{-\frac{1}{2}} \int_{1}^{\infty} e^{-4 \pi|m| v w} w^{-\frac{3}{2}} d w,
$$

where $H_{0}(m ; D)$ is given by $(8.29)$. 


\section{Derivatives of Eisenstein SERIES ANd Faltings heights}

Proof. We have

$$
\begin{aligned}
& \kappa(m, v)=\frac{1}{4} \int_{\Gamma \backslash D} \Xi(m, v) \cdot \mu \\
& =\frac{1}{4} \int_{\Gamma \backslash D} \sum_{x \in L(m)} \xi\left(v^{\frac{1}{2}} x, z\right) d \mu(z) \\
& =\frac{1}{4} \sum_{\substack{x \in L(m) \\
\bmod \Gamma}} \int_{\Gamma_{x} \backslash D} \xi\left(v^{\frac{1}{2}} x, z\right) d \mu(z) .
\end{aligned}
$$

First suppose that $m=Q(x)>0$, so $\Gamma_{x}$ is finite. Then

$$
\int_{\Gamma_{x} \backslash D} \xi(x, z) d \mu(z)=2\left|\Gamma_{x}\right|^{-1} \cdot \int_{D} \xi(x, z) d \mu(z)=4\left|\Gamma_{x}\right|^{-1} \cdot \int_{D^{+}} \xi(x, z) d \mu(z) .
$$

Here the factor of 2 occurs since $\Gamma_{x}$ contains \pm 1 , but these elements act trivially on $D$, while in the second step, we use the fact that $\xi(x, z)$ does not depend on the orientation of $z$. Since

$$
\xi(g x, g z)=\xi(x, z),
$$

for $g \in \mathrm{GL}_{2}(\mathbb{R})$, we may assume that

$$
x=m^{\frac{1}{2}} \cdot x_{0}=m^{\frac{1}{2}} \cdot\left(\begin{array}{cc} 
& 1 \\
-1 &
\end{array}\right) .
$$

Then, writing $z=k_{\theta}\left(e^{t} i\right) \in \mathfrak{H} \simeq D^{+}[\operatorname{Kud} 97 \mathrm{~b}$, p. 601], we have

$$
R(x, z)=2 m \sinh ^{2}(t) .
$$

Now, noting that $t$ runs from 0 to $\infty$ and $\theta$ runs from 0 to $\pi$,

$$
\begin{aligned}
I & :=\int_{D^{+}}-\operatorname{Ei}\left(-2 \pi R\left(v^{\frac{1}{2}} x, z\right)\right) d \mu(z) \\
& =\frac{1}{2 \pi} \int_{0}^{\pi} \int_{0}^{\infty}-\operatorname{Ei}\left(-4 \pi m v \sinh ^{2}(t)\right) 2 \sinh (t) d t d \theta \\
& =\frac{1}{2} \int_{0}^{\infty}\left(\int_{1}^{\infty} e^{-4 \pi m v \sinh ^{2}(t) r} r^{-1} d r\right) 2 \sinh (t) d t \\
& =\frac{1}{2} \int_{0}^{\infty}\left(\int_{1}^{\infty} e^{-4 \pi m v w r} r^{-1} d r\right)(w+1)^{-\frac{1}{2}} d w .
\end{aligned}
$$

However,

$$
\begin{aligned}
\int_{0}^{\infty} e^{-4 \pi m v w r}(w+1)^{-\frac{1}{2}} d w & =\left.e^{-4 \pi m v w r} 2(w+1)^{\frac{1}{2}}\right|_{0} ^{\infty}+4 \pi m v r \int_{0}^{\infty} e^{-4 \pi m v w r} 2(w+1)^{\frac{1}{2}} d w \\
& =-2+4 \pi m v r \int_{0}^{\infty} e^{-4 \pi m v w r} 2(w+1)^{\frac{1}{2}} d w \\
& =8 \pi m v r \int_{0}^{\infty} e^{-4 \pi m v w r}\left[(w+1)^{\frac{1}{2}}-1\right] d w,
\end{aligned}
$$

so that

$$
\begin{aligned}
I & =\frac{1}{2} \int_{1}^{\infty} 8 \pi m v \int_{0}^{\infty} e^{-4 \pi m v w r}\left[(w+1)^{\frac{1}{2}}-1\right] d w d r \\
& =\int_{0}^{\infty} e^{-4 \pi m v w}\left[(w+1)^{\frac{1}{2}}-1\right] w^{-1} d w \\
& =J(4 \pi m v) .
\end{aligned}
$$


By Lemma 9.2, we have

$$
\sum_{\substack{x \in L(m) \\ \bmod \Gamma}}\left|\Gamma_{x}\right|^{-1}=\delta(d ; D) \cdot H_{0}(m ; D) .
$$

Collecting terms, we obtain part i.

Next suppose that $m<0$. Let $\Gamma^{+}=\Gamma \cap \mathrm{GL}_{2}(\mathbb{R})^{+}$and let $\delta_{x}=\left|\Gamma_{x}: \Gamma_{x}^{+}\right|$, where $\Gamma_{x}^{+}=\Gamma_{x} \cap \Gamma^{+}$. Then

$$
\int_{\Gamma_{x} \backslash D} \xi(x, z) d \mu(z)=\delta_{x}^{-1} \int_{\Gamma_{x}^{+} \backslash D} \xi(x, z) d \mu(z)=2 \delta_{x}^{-1} \int_{\Gamma_{x}^{+} \backslash D^{+}} \xi(x, z) d \mu(z) .
$$

By conjugating by a suitable $g \in \mathrm{GL}_{2}(\mathbb{R})$, we can take

$$
g \cdot x=|m|^{\frac{1}{2}} \cdot x_{0}=|m|^{\frac{1}{2}} \cdot\left(\begin{array}{cc}
1 & \\
& -1
\end{array}\right) .
$$

Let $\Gamma^{\prime}$ be the corresponding conjugate of $\Gamma^{+}$in $\mathrm{SL}_{2}(\mathbb{R})$, and note that $\Gamma_{g x}^{\prime}$ will then be generated by $\pm 1_{2}$ and a unique element

$$
\left(\begin{array}{cc}
\epsilon(x) & \\
& \epsilon(x)^{-1}
\end{array}\right)
$$

for $\epsilon(x)>1$, the fundamental unit of norm 1 in the order $i_{x}^{-1}\left(O_{B}\right)$. If we write $z=r e^{i \theta} \in \mathfrak{H} \simeq D^{+}$, then $\Gamma_{g x}^{\prime}$ acts by multiplication by powers of $\epsilon(x)^{2}$. Note that

$$
R(g x, z)=\frac{2|m|}{\sin ^{2}(\theta)}
$$

Then

$$
\begin{aligned}
\int_{\Gamma_{x}^{+} \backslash D^{+}} \xi\left(v^{\frac{1}{2}} x, z\right) d \mu(z) & =\frac{1}{2 \pi} \int_{1}^{\epsilon(x)^{2}} \int_{0}^{\pi}-\operatorname{Ei}\left(-\frac{4 \pi|m| v}{\sin (\theta)^{2}}\right) r^{-1}(\sin (\theta))^{-2} d \theta d r \\
& =\frac{2}{\pi} \log |\epsilon(x)| \cdot \int_{0}^{\pi / 2}-\operatorname{Ei}\left(-\frac{4 \pi|m| v}{\sin (\theta)^{2}}\right)(\sin (\theta))^{-2} d \theta \\
& =\frac{1}{\pi} \log |\epsilon(x)| \cdot \int_{1}^{\infty}\left(\int_{1}^{\infty} e^{-4 \pi|m| v t w} w^{-1} d w\right)(t-1)^{-\frac{1}{2}} d t \\
& =\frac{1}{\pi} \log |\epsilon(x)| \cdot \int_{1}^{\infty} e^{-4 \pi|m| v w}\left(\int_{0}^{\infty} e^{-4 \pi|m| v t w} t^{-\frac{1}{2}} d t\right) w^{-1} d w \\
& =\frac{1}{\pi} \log |\epsilon(x)| \cdot \Gamma\left(\frac{1}{2}\right)(4 \pi|m| v)^{-\frac{1}{2}} \int_{1}^{\infty} e^{-4 \pi|m| v w} w^{-\frac{3}{2}} d w \\
& =\frac{1}{2 \pi} \log |\epsilon(x)| \cdot(|m| v)^{-\frac{1}{2}} \int_{1}^{\infty} e^{-4 \pi|m| v w} w^{-\frac{3}{2}} d w .
\end{aligned}
$$

The analogue of Lemma 9.2 for $m<0$ is the following.

Lemma 12.2. If $m<0$, then

$$
\sum_{\substack{x \in L(m) \\ \bmod \Gamma}} 2 \delta_{x}^{-1} \log |\epsilon(x)|=4 \delta(d ; D) H_{0}(m ; D),
$$

where $H_{0}(m ; D)$ is as in $(8.29)$.

Proof. We proceed as in the proof of Lemma 9.2. Note that for $x$ of type $c, \Gamma_{x} \simeq O_{c^{2} d}^{\times}$and $\Gamma_{x}^{+} \simeq O_{c^{2} d}^{1}$, the subgroup of norm 1 elements, so that $\delta_{x}=\delta_{c}=\left|O_{c^{2} d}^{\times}: O_{c^{2} d}^{1}\right|$. Let $\epsilon\left(c^{2} d\right)$ be a fundamental unit 
in $O_{c^{2} d}$ with $\epsilon\left(c^{2} d\right)>1$. Note that $\epsilon^{+}\left(c^{2} d\right)=\epsilon\left(c^{2} d\right)^{\delta_{c}}$ is a generator of $O_{c^{2} d}^{1} / \pm 1$. Then we have

$$
\begin{aligned}
\sum_{\substack{x \in L(m) \\
\bmod \Gamma}} 2 \delta_{x}^{-1} \log |\epsilon(x)| & =\sum_{\substack{c \mid n \\
(c, D)=1}} 2 \delta_{c}^{-1}\left|\operatorname{Opt}\left(O_{c^{2}}, O_{B}\right)\right| \cdot \log \left|\epsilon^{+}\left(c^{2} d\right)\right| \\
& =2 \sum_{\substack{c \mid n \\
(c, D)=1}}\left|\operatorname{Opt}\left(O_{c^{2} d}, O_{B}\right)\right| \cdot \log \left|\epsilon\left(c^{2} d\right)\right| \\
& =2 \delta(d ; D) \sum_{\substack{c \mid n \\
(c, D)=1}} h(d) \cdot \frac{\log |\epsilon(d)|}{\log \left|\epsilon\left(c^{2} d\right)\right|} \cdot\left(c \prod_{\ell \mid c}\left(1-\chi_{d}(\ell) \ell^{-1}\right)\right) \log \left|\epsilon\left(c^{2} d\right)\right| \\
& =4 \delta(d ; D) \frac{h(d) \log |\epsilon(d)|}{w(d)} \sum_{\substack{c \mid n \\
(c, D)=1}} c \prod_{\ell \mid c}\left(1-\chi_{d}(\ell) \ell^{-1}\right) \\
& =4 \delta(d ; D) H_{0}(m ; D) .
\end{aligned}
$$

This concludes the proof of Proposition 12.1.

Proof of Theorem 7.2 (concluded). We observe that the expression in Proposition 12.1 when $m>0$ coincides with the term (8.41) in the Fourier coefficient $\mathcal{E}_{m}^{\prime}\left(\tau, \frac{1}{2} ; D\right)=A_{m}^{\prime}\left(\frac{1}{2}, v\right) q^{m}$. On the other hand, when $m<0$, the expression in Proposition 12.1 coincides with that in part iii of Theorem 8.8.

\section{Part IV. Computations: analytic}

\section{Local Whittaker functions: the non-archimedean case}

The main purpose of this section is to prove Proposition 8.1. We fix a prime $p$ and frequently drop the subscript $p$ to lighten the notation. Recall that $B=B_{p}$ is a quaternion algebra which is a matrix algebra or a division algebra depending on whether $p \nmid D$ or $p \mid D$. Here $D$ is a fixed square-free positive integer. Let $O_{B}$ be a maximal order of $B$ and let

$$
V=\left\{x \in B \mid \operatorname{tr}^{0} x=0\right\}
$$

with the quadratic form $Q(x)=\kappa x^{2}$, where $\kappa= \pm 1$. Actually, only the case $\kappa=-1$ is needed in $\S 8$, but we treat the slightly more general case for future reference. Let $L=V \cap O_{B}$, and let $S \in \operatorname{Sym}_{2}\left(\mathbb{Q}_{p}\right)$ be the matrix associated with $L$ in the following sense. With respect to a basis of $L$ over $\mathbb{Z}_{p}$, identify $L$ with $\mathbb{Z}_{p}^{3}$. Then, for any $x \in L=\mathbb{Z}_{p}^{3}$,

$$
Q(x)=\frac{1}{2}(x, x)={ }^{t} x S x .
$$

Let

$$
S_{r}=S \perp \frac{1}{2}\left(\begin{array}{cc}
0 & I_{r} \\
I_{r} & 0
\end{array}\right),
$$

and let $L_{r}=\mathbb{Z}_{p}^{2 r+3}$ be the associated quadratic lattice, viewed as the direct sum of $L$ and $r$ hyperbolic planes. Let $d x=\prod d x_{i}$ be the standard Haar measure on $L_{r}$, where

$$
\int_{\mathbb{Z}_{p}} d x_{i}=1
$$

Let

$$
W\left(m, S_{r}\right)=\int_{\mathbb{Q}_{p}} \int_{L_{r}} \psi\left(b^{t} x S_{r} x\right) \psi(-m b) d x d b
$$




\section{S. S. Kudla, M. RAPOPORT And T. YANG}

be the integral defined in [Yan98, (1.2)]. It is the same as the local quadratic density polynomial $\alpha_{p}(X, m, S)$ with $X=p^{-r}$ defined in [Yan98, p. 312]. Here $\psi=\psi_{p}$ is the local component of our standard additive character of $\mathbb{A} / \mathbb{Q}$.

Lemma 13.1. With the notation as above, for any $r \geqslant 0$ and any $m \in \mathbb{Q}_{p}$,

$$
W_{m, p}\left(1, r+\frac{1}{2}, \Phi_{p}\right)=\beta(V)|\operatorname{det} 2 S|_{p}^{\frac{1}{2}} W\left(m, S_{r}\right) .
$$

Here

$$
\beta(V)=\left(\epsilon(S) \gamma(\psi)^{3} \gamma(\operatorname{det} S, \psi)\right)^{-1}
$$

is the local splitting index defined in [Kud92, Theorem 3.1]. Here $\epsilon(S)$ is the Hasse invariant ${ }^{6}$ of $S$ and $\gamma(\psi)$ and $\gamma(a, \psi)$ are the local Weil indices as in [Rao93].

Proof. We remark that this lemma is true in general for any three-dimensional quadratic space $V$ over $\mathbb{Q}_{p}$. Let $\phi=\operatorname{char}(L)$ and $\phi_{r}=\operatorname{char}\left(L_{r}\right)$. Then [Kud97b, Appendix], asserts that

$$
\omega(g) \phi_{r}(0)=\Phi_{p}\left(g, r+\frac{1}{2}\right)
$$

where $\omega=\omega_{\psi}$ is the Weil representation of $G_{p}^{\prime}$ (the metaplectic cover of $\mathrm{SL}_{2}\left(\mathbb{Q}_{p}\right)$ ) on the space $S(V)$ of Schwartz functions on $V$. Thus

$$
\begin{aligned}
W_{m, p}\left(1, r+\frac{1}{2}, \Phi_{p}\right) & =\int_{\mathbb{Q}_{p}} \omega(w n(b)) \phi_{r}(0) \psi(-b m) d b \\
& =\beta(V) \int_{\mathbb{Q}_{p}} \int_{V} \phi_{r}(x) d_{r} x \psi(-b m) d b,
\end{aligned}
$$

where $d_{r} x$ is the self-dual Haar measure with respect to the bi-character $(x, y) \mapsto \psi_{p}\left((x, y)_{r}\right)$ with $(x, y)_{r}$ the bilinear from on $V_{r}=L_{r} \otimes \mathbb{Q}_{p}$ associated to $S_{r}$. It is easy to check that

$$
d_{r} x=\left|\operatorname{det} 2 S_{r}\right|_{p}^{\frac{1}{2}} d x=|\operatorname{det} 2 S|_{p}^{\frac{1}{2}} d x
$$

under the identification $L_{r}=\mathbb{Z}_{p}^{2 r+3}$ as above. This proves the lemma.

The following lemma is standard.

Lemma 13.2. Let $V$ and $L$ be as above.

i) When $p \nmid D$, there is an isomorphism $L \cong \mathbb{Z}_{p}^{3}$ with the quadratic form and symmetric matrix as follows:

$$
Q(x)=\kappa\left(x_{1}^{2}+x_{2} x_{3}\right), \quad S=\kappa\left(\begin{array}{lll}
1 & & \\
& & \frac{1}{2} \\
& \frac{1}{2} &
\end{array}\right) .
$$

ii) When $2 \neq p \mid D$, there is an isomorphism $L \cong \mathbb{Z}_{p}^{3}$ with the quadratic form and symmetric matrix as follows:

$$
Q(x)=\kappa\left(\beta x_{1}^{2}+p x_{2}^{2}-\beta p x_{3}^{2}\right), \quad S=\kappa \operatorname{diag}(\beta, p,-\beta p) .
$$

Here $\beta \in \mathbb{Z}_{p}^{\times}$with $(\beta, p)_{p}=-1$.

iii) When $p=2 \mid D(B)$, there is an isomorphism $L \cong \mathbb{Z}_{p}^{3}$ with the quadratic form and symmetric matrix as follows:

$$
Q(x)=\kappa\left(x_{1}^{2}+x_{2}^{2}+x_{3}^{2}\right), \quad S=-\kappa \operatorname{diag}(1,1,1) .
$$

${ }^{6}$ If $S \in \operatorname{Sym}_{m}\left(\mathbb{Q}_{p}\right)$ is $\mathrm{GL}_{m}\left(\mathbb{Q}_{p}\right)$ equivalent to the $\operatorname{diagonal}$ form $\operatorname{diag}\left(a_{1}, \ldots, a_{m}\right)$, then

$$
\epsilon(S)=\prod_{i<j}\left(a_{i}, a_{j}\right)
$$




\section{DeRivatives of Eisenstein SERIES AND FALtings Heights}

iv) Finally,

$$
|\operatorname{det} 2 S|_{p}=|2 \kappa|_{p} \cdot \begin{cases}1 & \text { if } p \nmid D, \\ p^{-2} & \text { if } p \mid D .\end{cases}
$$

Note that $\beta(V)$ in Lemma 13.1 depends only on $V$ and is well defined even when $p=\infty$.

Lemma 13.3. Let $V$ be as above. Let $\zeta_{8}=e^{2 \pi i / 8}$. Then

$$
\beta(V)=\operatorname{inv}(B) \cdot \begin{cases}1 & \text { if } p \nmid 2 \infty, \\ \zeta_{8}^{-\kappa} & \text { if } p=\infty, \\ \zeta_{8}^{\kappa} & \text { if } p=2 .\end{cases}
$$

Here $\epsilon(B)= \pm 1$ depending on whether $B=B_{p}$ is split or not.

Proof. First, it is easy to check that the quantity

$$
\epsilon(\alpha S) \gamma\left(\psi_{\alpha}\right)^{3} \gamma\left(\operatorname{det}(\alpha S), \psi_{\alpha}\right)
$$

is independent of $\alpha \in \mathbb{Q}_{p}^{\times}$, so that we may write

$$
\beta(V)=\left(\epsilon(V) \gamma\left(\frac{1}{2} \psi\right)^{3} \gamma\left(\operatorname{det} V, \frac{1}{2} \psi\right)\right)^{-1},
$$

where $\operatorname{det}(V)=\operatorname{det}(2 S)$, as in [Rao93]. By Lemma 13.2, $\operatorname{det} V \in-2 \kappa \mathbb{Q}_{p}^{\times, 2}$ in all cases, and so, by [Rao93],

$$
\gamma\left(\operatorname{det} V, \frac{1}{2} \psi\right)=\gamma\left(-2 \kappa, \frac{1}{2} \psi\right)=\frac{\gamma(-\kappa \psi)}{\gamma\left(\frac{1}{2} \psi\right)} .
$$

Now we take $p=2$ and leave the other cases to the reader. Following [Rao93, p. 370], we have

$$
\gamma\left(\frac{1}{2} \psi\right)=\frac{1}{2} \sum_{x \in \mathbb{Z} / 4 \mathbb{Z}} \psi\left(\frac{1}{8} x^{2}\right)=\zeta_{8}^{-1},
$$

and [Rao93, Proposition A.12],

$$
\gamma(-\kappa \psi)=\zeta_{8}^{\kappa}
$$

Thus

$$
\gamma\left(\operatorname{det}(V), \frac{1}{2} \psi\right)= \begin{cases}i & \text { if } \kappa=1, \\ 1 & \text { if } \kappa=-1 .\end{cases}
$$

As for the Hasse invariant $\epsilon(V)=\epsilon(2 S)$, in the split case,

$$
\epsilon(V)=(2 \kappa,-1)_{2}(2 \kappa,-2 \kappa)_{2}=\left\{\begin{aligned}
1 & \text { if } \kappa=1, \\
-1 & \text { if } \kappa=-1 .
\end{aligned}\right.
$$

In the ramified case,

$$
\epsilon(V)=(-2 \kappa,-2 \kappa)_{2}=\left\{\begin{aligned}
-1 & \text { if } \kappa=1, \\
1 & \text { if } \kappa=-1 .
\end{aligned}\right.
$$

Combining these formulas, we obtain the claimed value.

Proof of Proposition 8.1. By Lemmas 13.1 and 13.3, Proposition 8.1 is equivalent to the following proposition for $\kappa=-1$.

Proposition 13.4. For a non-zero integer $m$, write $4 m=n^{2} d$ such that $\kappa d$ is a fundamental discriminant of a quadratic field. 
i) If $p \nmid D$,

$$
W_{p}\left(m, S_{r}\right)=\frac{L_{p}\left(r+1, \chi_{-\kappa d}\right) b_{p}(n, r+1 ; D)}{\zeta_{p}(2 r+2)}
$$

ii) If $p \mid D$,

$$
W_{p}\left(m, S_{r}\right)=L_{p}\left(r+1, \chi_{-\kappa d}\right) b_{p}(n, r+1 ; D) .
$$

iii) If $m=0$,

$$
W_{p}\left(0, S_{r}\right)= \begin{cases}\frac{\zeta_{p}(2 r+1)}{\zeta_{p}(2 r+2)} & \text { if } p \nmid D, \\ \frac{\zeta_{p}(2 r+1)}{\zeta_{p}(2 r)} & \text { if } p \mid D .\end{cases}
$$

Proof. Part i is a better reformulation of [Yan98, Propositions 8.3]. Part ii follows from i and [Yan98, Proposition 8.2]. Part iii follows from i and ii when we let $a=\operatorname{ord}_{p} m$ tend to infinity. We verify the case $p=2 \nmid D$ and leave the other (easier) cases to the reader. We recall again that $W_{p}\left(m, S_{r}\right)$ is just the local density polynomial $\alpha_{p}(X, m, S)$ in [Yan98] with $X=p^{-r}$. Write $m=\alpha p^{a}$ with $a=\operatorname{ord}_{p} m=2 k+\operatorname{ord}_{p}(d / 4)$ and $\alpha \in \mathbb{Z}_{p}^{\times}$where $k=k_{p}(n)=\operatorname{ord}_{p} n$ as before. In the notation of [Yan98, Proposition 8.3], we have $\left(\frac{\alpha \kappa}{p}\right)=\chi_{-\kappa d}(p)$ if $p \nmid d$. In our case $p=2 \nmid D$, $a=\operatorname{ord}_{2} m=2 k-2+\operatorname{ord}_{2} d$ with $k=\operatorname{ord}_{2} n$ as before.

Subcase 1. First we assume $8 \mid d$. Then $a=2 k+1$ is odd, and [Yan98, Proposition 8.3(1)], implies

$$
\begin{aligned}
W_{2}\left(m, S_{r}\right) & =\left(1-2^{-2} X^{2}\right) \sum_{l=0}^{k}\left(2^{-1} X^{2}\right)^{l} \\
& =\frac{\left(1-2^{-2} X^{2}\right)\left(1-\left(2^{-1} X^{2}\right)^{k+1}\right)}{1-2^{-1} X^{2}} \\
& =\frac{L_{2}\left(r+1, \chi_{-\kappa d}\right) b_{2}(n, r+1 ; D)}{\zeta_{2}(2 s+2)}
\end{aligned}
$$

as desired.

Subcase 2. Now assume that $\operatorname{ord}_{2} d=2$. Then $a=2 k, \alpha \kappa=(\kappa d / 4)\left(n 2^{-k}\right)^{2} \equiv-1 \bmod 4$, and thus $\left(\frac{-1}{\alpha \kappa}\right)=-1$ and $\delta_{8}(\alpha-\kappa)=0$. So [Yan98, Proposition 8.3(3)], $((a-1) / 2$ in the summation there should be $a / 2)$ implies

$$
\begin{aligned}
W_{2}\left(m, S_{r}\right) & =1+2^{-1} \sum_{l=1}^{k}\left(2^{-1} X^{2}\right)^{l}-2^{-k-2} X^{2 k+2} \\
& =\frac{\left(1-2^{-2} X^{2}\right)\left(1-\left(2^{-1} X^{2}\right)^{k+1}\right)}{1-2^{-1} X^{2}} \\
& =\frac{L_{2}\left(r+1, \chi_{-\kappa d}\right)}{\zeta_{2}(2 r+2)} b_{2}(n, r+1 ; D)
\end{aligned}
$$

Subcase 3. Finally if $2 \nmid d$, i.e. $\kappa d \equiv 1 \bmod 4$, then $a=2 k-2$ and $\alpha=d\left(n 2^{-k+1}\right)^{2} \equiv d \bmod 8$. In this case, $\left(\frac{-1}{\alpha \kappa}\right)=1$ and

$$
\delta_{8}(\alpha-\kappa)=\delta_{8}(d-\kappa)=\chi_{-\kappa d}(2)
$$




\section{Derivatives of Eisenstein SERIES ANd Faltings heights}

Set $v_{2}=\chi_{-\kappa d}(2)$. Then [Yan98, Proposition 8.3(3)] gives

$$
\begin{aligned}
W_{2}\left(m, S_{r}\right) & =1+2^{-1} \sum_{l=1}^{k-1}\left(2^{-1} X^{2}\right)^{l}+2^{-k-1} X^{2 k}+v_{2} 2^{-k-1} X^{2 k+1} \\
& =\frac{1-2^{-2} X^{2}-2^{-k-1} X^{2 k+1}\left(-v_{2}+2^{-1} X+v_{2} 2^{-1} X^{2}\right)}{1-2^{-1} X^{2}} .
\end{aligned}
$$

On the other hand,

$$
\begin{aligned}
\frac{L_{2}\left(r+1, \chi_{-\kappa d}\right) b_{2}(n, r+1 ; D)}{\zeta_{2}(2 r+2)} & =\frac{\left(1+v_{2} 2^{-1} X\right)\left(1-v_{2} X+v_{2} 2^{-k-2} X^{2 k+1}-2^{-k-1} X^{2 k+2}\right)}{1-2^{-1} X^{2}} \\
& =\frac{1-2^{-2} X^{2}-2^{-k-1} X^{2 k+1}\left(1+v_{2} 2^{-1} X\right)\left(-v_{2}+X\right)}{1-2^{-1} X^{2}} \\
& =\frac{1-2^{-2} X^{2}-2^{-k-1} X^{2 k+1}\left(-v_{2}+2^{-1} X+v_{2} 2^{-1} X^{2}\right)}{1-2^{-1} X^{2}}
\end{aligned}
$$

Therefore

$$
W_{2}\left(m, S_{r}\right)=\frac{L_{2}\left(r+1, \chi_{-\kappa d}\right)}{\zeta_{2}(2 r+2)} b_{2}(n, r+1 ; D) .
$$

This concludes the proof of Proposition 8.1.

\section{Local Whittaker functions: the archimedean case}

In this section, we compute the local Whittaker function

$$
W_{m, \infty}\left(\tau, s, \Phi_{\infty}^{\ell}\right)=v^{-\ell / 2} \int_{\mathbb{R}} \Phi_{\infty}^{\ell}\left(w n(b) g_{\tau}^{\prime}, s\right) \psi_{\infty}(-m b) d b
$$

and prove Lemmas 8.9 and 8.11 . Here $\ell \in \frac{1}{2} \mathbb{Z}$ is such that $\ell \equiv \frac{3}{2} \bmod 2 \mathbb{Z}$. In this paper, we only need $\ell=\frac{3}{2}$.

Let

$$
\Psi(a, b ; z)=\frac{1}{\Gamma(a)} \int_{0}^{\infty} e^{-z r}(r+1)^{b-a-1} r^{a-1} d r
$$

be the standard confluent hypergeometric function of the second kind [Leb72], where $a>0, z>0$ and $b$ is any real number. It satisfies the functional equation [Leb72, p. 265]

$$
\Psi(a, b ; z)=z^{1-b} \Psi(1+a-b, 2-b ; z) .
$$

For convenience, we also define

$$
\Psi(0, b ; z)=\lim _{a \rightarrow 0+} \Psi(a, b ; z)=1 .
$$

So $\Psi(a, b ; z)$ is well defined for $z>0, a \geqslant \min \{0, b-1\}$. Finally, for any number $n$, we define

$$
\Psi_{n}(s, z)=\Psi\left(\frac{1}{2}(1+n+s), s+1 ; z\right) .
$$

Then (14.3) implies

$$
\Psi_{n}(s, z)=z^{-s} \Psi_{n}(-s, z) .
$$

Proposition 14.1. Let $q=e(m \tau),(-i)^{\ell}=e(-\ell / 4)$, and

$$
\alpha=\frac{s+1+\ell}{2}, \quad \beta=\frac{s+1-\ell}{2} .
$$




\section{S. S. Kudla, M. Rapoport And T. YAnG}

i) For $m>0$,

$$
W_{m, \infty}\left(\tau, s, \Phi_{\infty}^{\ell}\right)=2 \pi(-i)^{\ell} v^{\beta}(2 \pi m)^{s} \frac{\Psi_{-\ell}(s, 4 \pi m v)}{\Gamma(\alpha)} \cdot q^{m}
$$

ii) For $m<0$,

$$
W_{m, \infty}\left(\tau, s, \Phi_{\infty}^{\ell}\right)=2 \pi(-i)^{\ell} v^{\beta}(2 \pi|m|)^{s} \frac{\Psi_{\ell}(s, 4 \pi|m| v)}{\Gamma(\beta)} e^{-4 \pi|m| v} \cdot q^{m}
$$

iii) For $m=0$,

$$
W_{0, \infty}\left(\tau, s, \Phi_{\infty}^{\ell}\right)=2 \pi(-i)^{\ell} v^{\frac{1}{2}(1-\ell-s)} \frac{2^{-s} \Gamma(s)}{\Gamma(\alpha) \Gamma(\beta)} .
$$

iv) The special value at $s=\ell-1$ is

$$
W_{m, \infty}\left(\tau, \ell-1, \Phi_{\infty}^{\ell}\right)= \begin{cases}0 & \text { if } m \leqslant 0 \\ \frac{(-2 \pi i)^{\ell}}{\Gamma(\ell)} m^{\ell-1} q^{m} & \text { if } m>0 .\end{cases}
$$

Proof. A standard calculation [Shi82] (see also [Kud97b, pp. 585-586], for this special case) gives

$$
W_{m, \infty}\left(\tau, s, \Phi_{\infty}^{\ell}\right)=(-i)^{\ell} v^{\beta} e(m \bar{\tau}) \frac{(2 \pi)^{s+1}}{\Gamma(\alpha) \Gamma(\beta)} \int_{\substack{r>0 \\ r>m}} e^{-4 \pi v r}(r-m)^{\beta-1} r^{\alpha-1} d r .
$$

When $m=0$, this immediately gives part iii.

When $m>0$, the integral equals

$$
\begin{aligned}
\int_{r>m} e^{-4 \pi v r}(r-m)^{\beta-1} r^{\alpha-1} d r & =m^{s} e^{-4 \pi m v} \int_{0}^{\infty} e^{-4 \pi m v r} r^{\beta-1}(r+1)^{\alpha-1} d r \\
& =m^{s} e^{-4 \pi m v} \Gamma(\beta) \Psi_{-\ell}(s, 4 \pi m v) .
\end{aligned}
$$

This proves part $\mathrm{i}$. The special value at $s=\ell-1$ is

$$
\begin{aligned}
W_{m, \infty}\left(\tau, \ell-1, \Phi_{\infty}^{\ell}\right) & =2 \pi(-i)^{\ell}(2 \pi m)^{\ell-1} q^{m} \frac{\Psi_{-\ell}(\ell-1,4 \pi m v)}{\Gamma(\ell)} \\
& =\frac{(-2 \pi i m)^{\ell}}{m \Gamma(\ell)} q^{m},
\end{aligned}
$$

as claimed in part iv.

When $m<0$, the integral is

$$
\begin{aligned}
\int_{r>0} e^{-4 \pi v r}(r-m)^{\beta-1} r^{\alpha-1} d r & =|m|^{s} \int_{0}^{\infty} e^{-4 \pi m v r} r^{\alpha-1}(r+1)^{\beta-1} d r \\
& =|m|^{s} \Gamma(\alpha) \Psi_{\ell}(s, 4 \pi m v) .
\end{aligned}
$$

This proves part ii. The special value at $s=\ell-1$ is zero since $1 / \Gamma(\beta)=0$ at $s=\ell-1$ and $\Psi_{\ell}(\ell-1,4 \pi|m| v)$ is finite.

Proof of Lemma 8.9. Since $m>0$, part i of Proposition 14.1 implies

$$
\frac{W_{m, \infty}^{\prime}\left(\tau, \ell-1, \Phi_{\infty}^{\ell}\right)}{W_{m, \infty}\left(\tau, \ell-1, \Phi_{\infty}^{\ell}\right)}=\frac{1}{2} \log v+\log (2 \pi m)-\frac{1}{2} \frac{\Gamma^{\prime}(\ell)}{\Gamma(\ell)}+\frac{\Psi_{-\ell}^{\prime}(\ell-1,4 \pi m v)}{\Psi_{-\ell}(\ell-1,4 \pi m v)} .
$$

Note that, for any $z>0$,

$$
\Psi_{-\ell}(\ell-1, z)=\Psi(0, \ell ; z)=1
$$

by (14.4). Observe that

$$
\Psi_{-\ell}(s, z)=z^{-\beta}+\frac{1}{\Gamma(\beta)} \int_{0}^{\infty} e^{-z r}\left((r+1)^{s-\beta}-1\right) r^{\beta-1} d r .
$$




\section{DeRivatives of Eisenstein SERIES AND FALtings Heights}

The integral here is well-defined at $s=\ell-1$ and is equal to

$$
J(\ell-1, z):=\int_{0}^{\infty} e^{-z r} \frac{(r+1)^{\ell-1}-1}{r} d r .
$$

Note also that the function $1 / \Gamma(\beta)$ vanishes at $s=\ell-1$ and has the first derivative $\frac{1}{2}$ at $s=\ell-1$. Thus,

and we have

$$
\Psi_{-\ell}^{\prime}(\ell-1, z)=-\frac{1}{2} \log z+\frac{1}{2} J(\ell-1, z)
$$

$$
\frac{W_{m, \infty}^{\prime}\left(\tau, \ell-1, \Phi_{\infty}^{\ell}\right)}{W_{m, \infty}\left(\tau, \ell-1, \Phi_{\infty}^{\ell}\right)}=\frac{1}{2}\left[\log (\pi m)-\frac{\Gamma^{\prime}(\ell)}{\Gamma(\ell)}+J(\ell-1,4 \pi m v)\right] .
$$

When $\ell=\frac{3}{2}, J\left(\frac{1}{2}, 4 \pi m v\right)=J(4 \pi m v)$ is the quantity defined in Theorem 8.8, so this gives Lemma 8.9.

Proof of Lemma 8.11. (The case $m<0$, with derivative). We now assume $m<0$. Since the function $1 / \Gamma(\beta)$ vanishes at $s=\ell-1$ and has the first derivative $\frac{1}{2}$ there, we have, by part ii of Proposition 14.1,

$$
W_{m, \infty}^{\prime}\left(\tau, \ell-1, \Phi_{\infty}^{\ell}\right)=2 \pi(-i)^{\ell}(2 \pi|m|)^{\ell-1} \frac{1}{2} \Psi_{\ell}(\ell-1,4 \pi|m| v) \cdot e^{-4 \pi|m| v} \cdot q^{m} .
$$

By (14.6), we have

$$
\begin{aligned}
\Psi_{\ell}(\ell-1,4 \pi|m| v) & =(4 \pi|m| v)^{1-\ell} \Psi_{\ell}(1-\ell, 4 \pi|m| v) \\
& =(4 \pi|m| v)^{1-\ell} \Psi(1,2-\ell ; 4 \pi|m| v) \\
& =(4 \pi|m| v)^{1-\ell} \int_{0}^{\infty} e^{-4 \pi|m| v r}(1+r)^{-\ell} d r \\
& =(4 \pi|m| v)^{1-\ell} e^{4 \pi|m| v} \int_{1}^{\infty} e^{-4 \pi|m| v r} r^{-\ell} d r
\end{aligned}
$$

Therefore,

$$
W_{m, \infty}^{\prime}\left(\tau, \ell-1, \Phi_{\infty}^{\ell}\right)=2 \pi(-i)^{\ell} 2^{-\ell} v^{1-\ell} q^{m} \int_{1}^{\infty} e^{-4 \pi|m| v r} r^{-\ell} d r
$$

When $\ell=\frac{3}{2}$, this gives Lemma 8.11.

\section{The functional equation}

Let $D$ be a square-free positive integer, not necessarily the discriminant of an indefinite quaternion algebra, and let

$$
\mathbb{E}\left(\tau, s, \Phi^{\frac{3}{2}, D}\right)=c(D)\left(s+\frac{1}{2}\right) \Lambda_{D}(2 s+1) E\left(\tau, s, \Phi^{\frac{3}{2}, D}\right)
$$

be the renormalized Eisenstein series of (6.23). In this section, we prove that it is invariant when $s$ goes to $-s$, i.e. that

$$
\mathbb{E}\left(\tau, s, \Phi^{\frac{3}{2}, D}\right)=\mathbb{E}\left(\tau,-s, \Phi^{\frac{3}{2}, D}\right) .
$$

First we need the following.

Proposition 15.1. Set

$$
\Lambda\left(s, \chi_{m} ; D\right)=\left(\frac{4|m| D^{2}}{\pi}\right)^{\frac{1}{2} s} \Gamma\left(\frac{s+a}{2}\right) L\left(s, \chi_{d}\right) \prod_{p} b_{p}(n, s, D)
$$

with $a=(1+\operatorname{sgn}(m)) / 2$. Then $\Lambda\left(s, \chi_{m}, D\right)$ has a meromorphic continuation to the whole complex $s$-plane with possible poles at $s=0$ and 1 , which occur precisely when $D=-d=1$. Furthermore, 


\section{S. S. Kudla, M. Rapoport And T. YAng}

it satisfies the following functional equation

$$
\Lambda\left(s, \chi_{m} ; D\right)=\Lambda\left(1-s, \chi_{m} ; D\right),
$$

and

$$
\operatorname{ord}_{s=0} \Lambda\left(s, \chi_{m} ; D\right)=\operatorname{ord}_{s=1} \Lambda\left(s, \chi_{m} ; D\right)=\operatorname{ord}_{s=1} L\left(s, \chi_{d}\right)+\#\left\{p|D| \chi_{d}(p)=1\right\}
$$

Proof. The functional equation follows from that of $L\left(s, \chi_{d}\right)$ and (8.10). The vanishing order at $s=0$ follows from (8.13). We remark that $b_{p}(n, s ; D)$ is a polynomial in $p^{-s}$ even though it was written as a rational function and thus is regular at $s=\frac{1}{2}$.

THEOREM 15.2. Let

$$
\mathbb{E}\left(\tau, s, \Phi^{\frac{3}{2}, D}\right)=\sum_{m} \mathcal{A}_{m}(v, s) q^{m}
$$

be the Fourier expansion of $\mathbb{E}\left(\tau, s, \Phi^{\frac{3}{2}, D}\right)$.

i) For $m>0$,

$$
\mathcal{A}_{m}(v, s)=\frac{\Lambda\left(\frac{1}{2}+s, \chi_{m} ; D\right)(4 \pi m v)^{\frac{s}{2}-\frac{1}{4}} \Psi_{-\frac{3}{2}}(s, 4 \pi m v)}{\sqrt{\pi} \prod_{p \mid D}(1+p)} .
$$

ii) For $m<0$,

$$
\mathcal{A}_{m}(v, s)=\frac{\left(s^{2}-\frac{1}{4}\right) \Lambda\left(\frac{1}{2}+s, \chi_{m} ; D\right)(4 \pi|m| v)^{\frac{s}{2}-\frac{1}{4}} \Psi_{\frac{3}{2}}(s, 4 \pi|m| v)}{4 \sqrt{\pi} \prod_{p \mid D}(1+p)} \cdot e^{-4 \pi|m| v} .
$$

iii) The constant term is

$$
\mathcal{A}_{0}(v, s)=-\frac{D}{2 \pi \prod_{p \mid D}(p+1)}\left(G_{D}(s)+G_{D}(-s)\right),
$$

where

$$
G_{D}(s)=v^{-\frac{1}{4}+\frac{s}{2}} \Lambda(1+2 s)\left(s+\frac{1}{2}\right) \prod_{p \mid D}\left(p^{-\frac{1}{2}-s}-p^{\frac{1}{2}+s}\right) .
$$

Proof. When $m>0$, we have, by Propositions 8.1 and 14.1 and formula (8.16),

$$
\begin{aligned}
\mathcal{A}_{m}(v, s)= & c(D)\left(\frac{D}{\pi}\right)^{s-\frac{1}{2}} \Gamma\left(s+\frac{3}{2}\right) \zeta_{D}(2 s+1) \frac{C_{\infty}}{\sqrt{2}} v^{\frac{1}{2}\left(s-\frac{1}{2}\right)}(2 \pi m)^{s} \frac{\Psi_{-\frac{3}{2}}(s, 4 \pi m v)}{\Gamma\left(\frac{s}{2}+\frac{5}{4}\right)} \\
& \times C_{f}(D) \frac{L\left(s+\frac{1}{2}, \chi_{d}\right) \prod b_{p}\left(n, s+\frac{1}{2} ; D\right)}{\zeta_{D}(2 s+1)} \\
= & \frac{\Lambda\left(s+\frac{1}{2}, \chi_{m} ; D\right)}{\prod_{p \mid D}(p+1)} \frac{\Gamma\left(s+\frac{3}{2}\right) 2^{-s-\frac{1}{2}}}{\Gamma\left(\frac{s}{2}+\frac{3}{4}\right) \Gamma\left(\frac{s}{2}+\frac{5}{4}\right)}(4 \pi m v)^{\frac{1}{2}\left(s-\frac{1}{2}\right)} \Psi_{-\frac{3}{2}}(s, 4 \pi m v) .
\end{aligned}
$$

Now the doubling formula of the gamma function gives

$$
\frac{\Gamma\left(s+\frac{3}{2}\right)}{\Gamma\left(\frac{s}{2}+\frac{3}{4}\right) \Gamma\left(\frac{s}{2}+\frac{5}{4}\right)}=2^{s+\frac{3}{2}-1} \pi^{-\frac{1}{2}}=2^{s+\frac{1}{2}} \pi^{-\frac{1}{2}} .
$$

This proves part i. The case $m<0$ is the same and is left to the reader. When $m=0$, by 


\section{Derivatives of Eisenstein SERIES ANd FAltings Heights}

Corollary 8.2, Lemma 8.3 and (8.11), we have

$$
\begin{aligned}
\mathcal{A}_{0}(v, s)= & c(D)\left(\frac{D}{\pi}\right)^{s-\frac{1}{2}} \Gamma\left(s+\frac{3}{2}\right) \zeta_{D}(2 s+1) \\
& \times\left[v^{-\frac{1}{4}+\frac{s}{2}}+\frac{C_{\infty}}{\sqrt{2}} \frac{2^{-s} v^{-\frac{1}{4}-\frac{s}{2}} \Gamma(s)}{\Gamma\left(\frac{s}{2}-\frac{1}{4}\right) \Gamma\left(\frac{s}{2}+\frac{5}{4}\right)} \frac{C_{f}(D) \zeta(2 s)}{\zeta_{D}(2 s+1)} \prod_{p \mid D}\left(1-p^{1-2 s}\right)\right] \\
= & -\frac{v^{-\frac{1}{4}+\frac{s}{2}} \Lambda(1+2 s)\left(\frac{1}{2}+s\right)}{2 \pi \prod_{p \mid D}(p+1)}(-1)^{\operatorname{ord}(D)} D^{s+\frac{3}{2}} \prod_{p \mid D}\left(1-p^{-1-2 s}\right) \\
& -\frac{v^{-\frac{1}{4}-\frac{s}{2}} \Lambda(2 s)\left(\frac{1}{2}-s\right)}{\sqrt{\pi} \prod_{p \mid D}(p+1)} \frac{\Gamma\left(s-\frac{1}{2}\right) 2^{\frac{1}{2}-s}}{\Gamma\left(\frac{s}{2}-\frac{1}{4}\right) \Gamma\left(\frac{s}{2}+\frac{1}{4}\right)} D^{\frac{1}{2}+s} \prod_{p \mid D}\left(1-p^{1-2 s}\right) \\
= & -\frac{D}{2 \pi \prod_{p \mid D}(p+1)}\left(G_{D}(s)+G_{D}(-s)\right) .
\end{aligned}
$$

Here we have used the doubling formula for the gamma functions again.

Proof of the functional equation (15.2). Now the functional equation (15.2) follows immediately from Theorem 15.2, Proposition 15.1 and (14.6).

\section{ACKNOWLEDGEMENTS}

The results of this paper are an outgrowth of a project begun during the first author's visit to the Mathematische Institut of the University of Cologne in the autumn of 1999. He would like to thank the Institut for providing a congenial and stimulating working environment. The second author thanks the Department of Mathematics of the University of Maryland for its (by now almost customary) hospitality during his sabbatical in the spring of 2001.

\section{REFERENCES}

BS66 A. I. Borevich and I. R. Shafarevich, Number theory (Academic Press, New York, 1966).

BC91 J.-F. Boutot and H. Carayol, Uniformisation p-adique des courbes de Shimura, in Courbes Modulaires et Courbes de Shimura, Astérisque 196-197 (1991), 45-158.

BGS94 J.-B. Bost, H. Gillet and C. Soulé, Heights of projective varieties and positive Green forms, J. Amer. Math. Soc. 7 (1994), 903-1027.

Bos J.-B. Bost, unpublished.

Bos91 J.-B. Bost, Théorie de l'intersection et théorème de Riemann-Roch arithmétiques, in Sém. Bourbaki $n^{o}$ 731, Astérisque 201-203 (1991), 43-88.

Bos99 J.-B. Bost, Potential theory and Lefschetz theorems for arithmetic surfaces, Ann. Sci. École Norm. Sup. 32 (1999), 241-312.

CF90 C. L. Chai and G. Faltings, Degeneration of abelian varieties, Ergebnisse der Mathematik und ihrer Grenzgebiete, 3. Folge, vol. 22 (Springer, Berlin, 1990).

Coh75 H. Cohen, Sums involving the values at negative integers of L-functions of quadratic characters, Math. Ann. 217 (1975), 271-285.

Col93 P. Colmez, Périodes des variétés abéliennes à multiplication complexe, Ann. of Math. (2) 138 (1993), 625-683.

DM69 P. Deligne and D. Mumford, Irreducibility of the space of curves of a given genus, Publ. Math. IHES 36 (1969), 75-109. 


\section{S. S. Kudla, M. Rapoport And T. YAng}

DR73 P. Deligne and M. Rapoport, Les schémas de modules des courbes elliptiques, in Modular functions of one variable II, Antwerp, 1972, Lecture Notes in Mathematics, vol. 349 (Springer, Berlin, 1973), 143-316.

Dri77 V. G. Drinfeld, Coverings of p-adic symmetric regions, Funct. Anal. Appl. 10 (1977), 29-40.

Eic56 M. Eichler, Modular Correspondences (TATA Institute, Bombay, 1956).

Fal83 G. Faltings, Endlichkeitssätze für abelsche Varietäten über Zahlkörpern, Invent. Math. 73 (1983), 349-366.

Fun99 J. Funke, Rational quadratic divisors and automorphic forms, Thesis, University of Maryland (1999).

Fun02 J. Funke, Heegner divisors and non-holomorphic modular forms, Compositio Math. 133 (2002), 289-321.

Gel76 S. Gelbart, Weil's representation and the spectrum of the metaplectic group, Lecture Notes in Mathematics, vol. 530 (Springer, New York, 1976).

Gro86 B. H. Gross, On canonical and quasi-canonical liftings, Invent. Math. 84 (1986), 321-326.

GS90 H. Gillet and C. Soulé, Arithmetic intersection theory, Publ. Math. IHES 72 (1990), 93-174.

Hai00 R. Hain, Moduli of Riemann surfaces, transcendental aspects, ICTP Lecture Notes, vol. 1 (Abdus Salam Int. Cent. Theoret. Phys., Trieste, 2000), 293-353.

HZ76 F. Hirzebruch and D. Zagier, Intersection numbers of curves on Hilbert modular surfaces and modular forms of Nebentypus, Invent. Math. 36 (1976), 57-113.

Kot92 R. Kottwitz, Points on some Shimura varieties over finite fields, J. Amer. Math. Soc. 5 (1992), 373-444.

KR99 S. Kudla and M. Rapoport, Arithmetic Hirzebruch-Zagier cycles, J. reine angew. Math. 515 (1999), $155-244$.

KR00a S. Kudla and M. Rapoport, Height pairings on Shimura curves and p-adic unformization, Invent. Math. 142 (2000), 153-223.

KR00b S. Kudla and M. Rapoport, Cycles on Siegel threefolds and derivatives of Eisenstein series, Ann. Sci. École Norm. Sup. 33 (2000), 695-756.

KRY S. Kudla, M. Rapoport and T. Yang, in preparation.

KRY99 S. Kudla, M. Rapoport and T. Yang, On the derivative of an Eisenstein series of weight 1, Int. Math. Res. Notices 7 (1999), 347-385.

Kud92 S. Kudla, Splitting metaplectic covers of dual reductive pairs, Israel J. Math. 87 (1992), 361-401.

Kud97a S. Kudla, Algebraic cycles on Shimura varieties of orthogonal type, Duke Math. J. 86 (1997), 39-78.

Kud97b S. Kudla, Central derivatives of Eisenstein series and height pairings, Ann. of Math. (2) 146 (1997), 545-646.

Kud01 S. Kudla, Special cycles and derivatives of Eisenstein series, in Proc. of Conference on Special Values of Rankin L-series, MSRI, December 2001, to appear.

Kud02a S. Kudla, Derivatives of Eisenstein series and generating functions for arithmetic cycles, Sém. Bourbaki no 876, Astérisque 276 (2002), 341-368.

Kud02b S. Kudla, Eisenstein series and arithmetic geometry, in Proc. ICM, vol. II (Higher Ed. Press, Beijing, 2002), 173-183.

Kud03a S. Kudla, Integrals of Borcherds forms, Compositio Math. 137 (2003), 293-349.

Kud03b S. Kudla, Modular forms and arithmetic geometry, in Current Developments in Mathematics (International Press, Boston, 2003), 135-180.

Kuh01 U. Kühn, Generalized arithmetic intersection numbers, J. reine angew. Math. 534 (2001), 209-236.

KY S. Kudla and T. Yang, in preparation.

Leb72 N. N. Lebedev, Special functions and their applications (Dover, New York, 1972).

NT91 Y. Nakkajima and Y. Taguchi, A generalization of the Chowla-Selberg formula, J. reine angew. Math. 419 (1991), 119-124.

Rao93 R. Rao, On some explicit formulas in the theory of Weil representations, Pacific J. Math. 157 (1993), 335-371. 


\section{Derivatives of Eisenstein series AND FAltings Heights}

Ray85 M. Raynaud, Hauteurs et isogénies, in Séminaire sur les pinceaux arithmétiques: la conjecture de Mordell, Astérisque 127, (1985), 199-234.

RZ96 M. Rapoport and T. Zink, Period spaces for p-divisible groups, Annals of Math. Studies vol. 141 (Princeton University Press, Princeton, NJ, 1996).

Shi82 G. Shimura, Confluent hypergeometric functions on tube domains, Math. Ann. 260 (1982), 269-302.

Vis89 A. Vistoli, Intersection theory on algebraic stacks and on their moduli spaces, Invent. Math. 97 (1989), 613-670.

Wal80 J.-L. Waldspurger, Correspondance de Shimura, J. Math. Pures Appl. 59 (1980), 1-132.

Wei64 A. Weil, Sur certains groupes d'opérateurs unitaires, Acta Math. 111 (1964), 143-211.

Yan T. H. Yang, The second term of an Eisenstein series, in Proc. of the 2nd International Congress of Chinese Mathematicians, to appear.

Yan98 T. H. Yang, An explicit formula for local densities of quadratic forms, J. Number Theory 72 (1998), 309-356.

Zag75 D. Zagier, Nombres de classes et formes modulaires de poids 3/2, C. R. Acad. Sci. Paris 281 (1975), 883-886.

Stephen S. Kudla ssk@math.umd.edu

Department of Mathematics, University of Maryland, College Park, MD 20742, USA

Michael Rapoport rapoport@uni-bonn.de

Mathematisches Institut der Universität Bonn, Beringstr. 1, 53115 Bonn, Germany

Tonghai Yang thyang@math.wisc.edu

Department of Mathematics, University of Wisconsin, Madison, WI 53706, USA 